\title{
Strontium-90 Fluoride Data Sheet
}

\author{
H. T. Fullam
}

June 1981

Prepared for the Office of Waste Operations and Technology U.S. Department of Energy under Contract DE-AC06-76RLO 1830 
NOT ICE

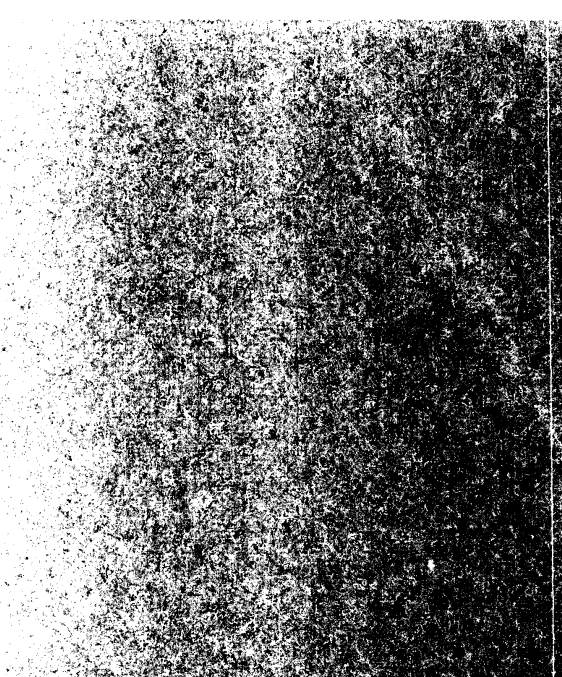

This report was prepared as an account of work sponsored by the United States Gover ond Wh nor the Department of Energy, nor any of their employees, nor any of their coptw

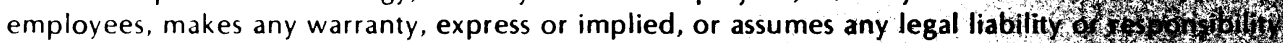
completeness or usefulness of any information, apparatus, product or process disclose $\mathrm{X}_{\mathrm{K}} \mathrm{W}$ not infringe privately owned rights.

The views, opinions and conclusions contained in this report are those of the chint represent those of the United States Government or the United States Department of ${ }_{1}$ 


\section{9}

PNL -3846

$U C-4,-23$

\section{STRONTIUM-90 FLUORIDE DATA SHEET}

H. T. Fullam

June 1981

Prepared for the Office of Waste Operations and Technology U. S. Department of Energy under Contract DE-AC06-76RLO 1830

Pacific Northwest Laboratory Richland, Washington 99352 


\section{ABSTRACT}

This report is a compilation of available data and appropriate literature references on the properties of strontium-90 fluoride and nonradioactive strontium fluoride. The objective of the document is to compile in a single source pertinent data to assist potential users in the development, licensing, and use of ${ }^{90}{ }^{S r F}$ 2-fueled radioisotope heat sources for terrestrial power conversion and thermal applications. The report is an update of the Strontium-90 Fluoride Data Sheet (BNWL-2284) originally issued in April 1977. 


\section{CONTENTS}

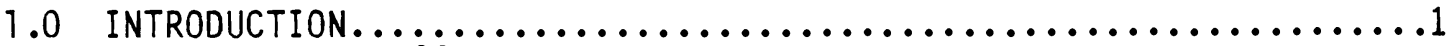

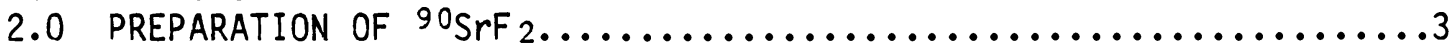

2.1 WESF FLOWSHEET FOR THE PREPARATION $0 \mathrm{~F}$ $9 \mathrm{O}_{\mathrm{S}} \mathrm{F}_{2} \ldots \ldots \ldots \ldots \ldots \ldots . \ldots$

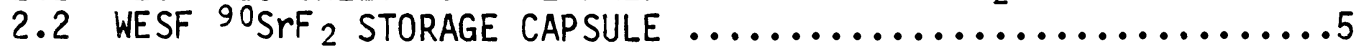

3.0 PROPERTIES OF STRONTIUM FLUORIDE......................

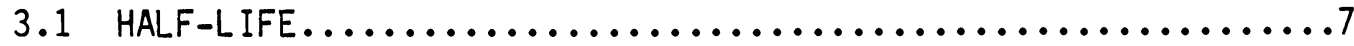

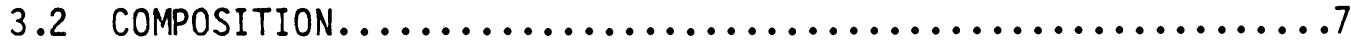

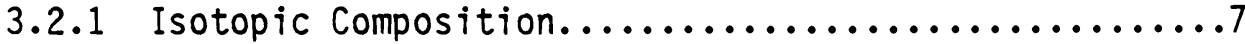

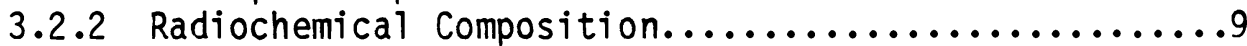

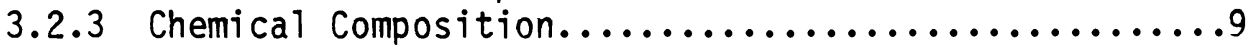

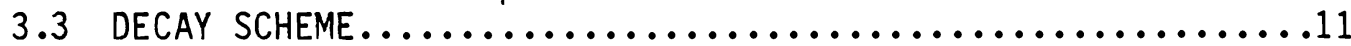

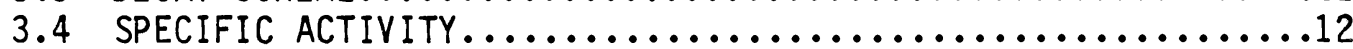

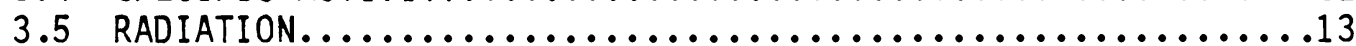

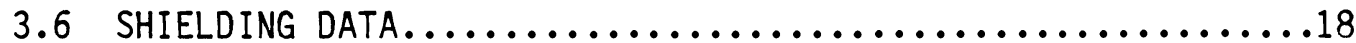

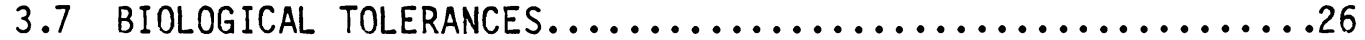

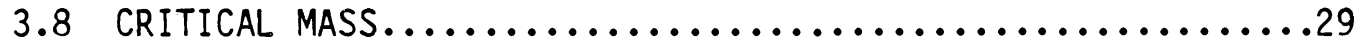

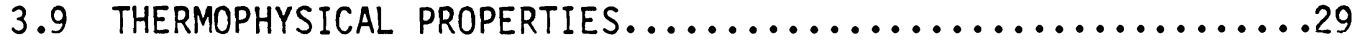

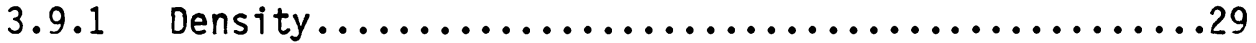

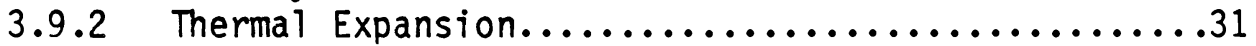

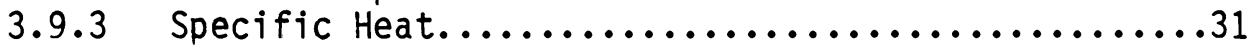

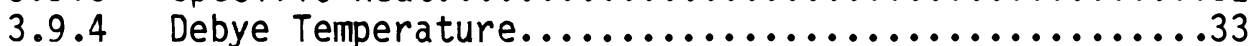

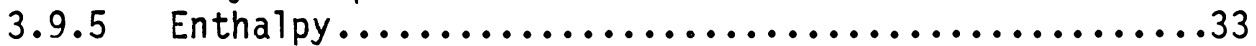

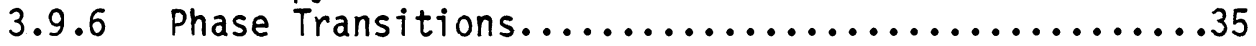

3.9.7 Latent Heats of Phase Transitions...............36

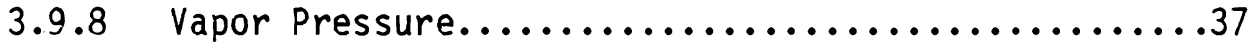

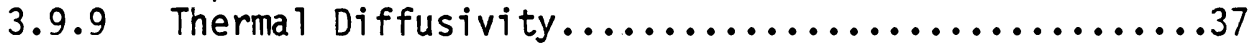

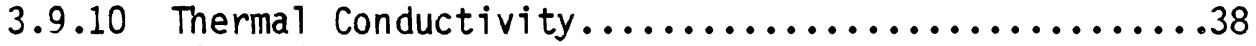

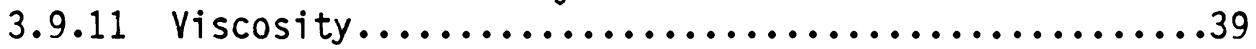

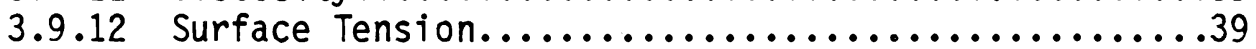

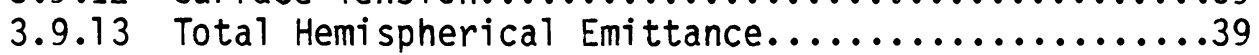

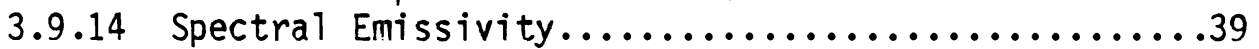

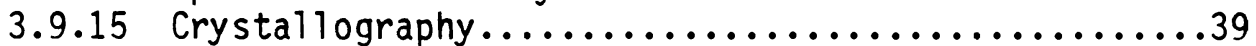

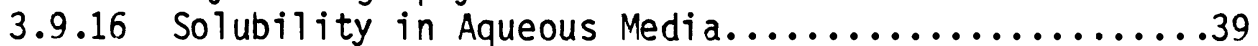

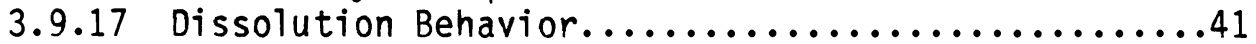

3.10 OPTICAL AND ELECTRONIC PROPERTIES.................43

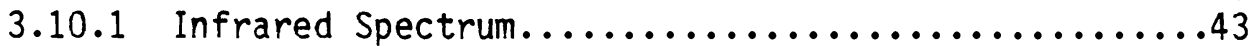

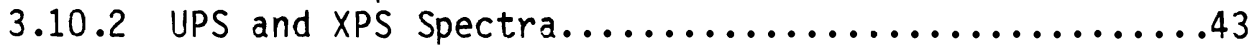

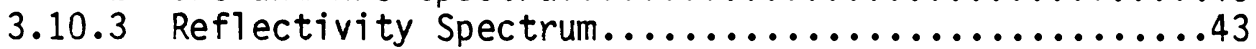

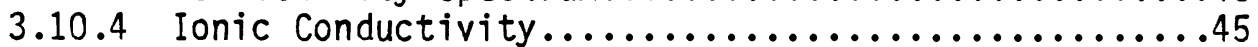

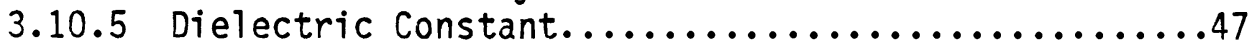

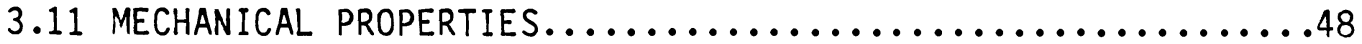

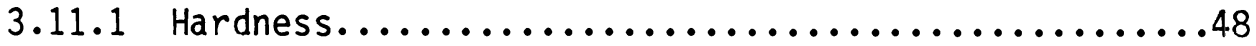

3.11 .2 Modulus of Rupture.......................48

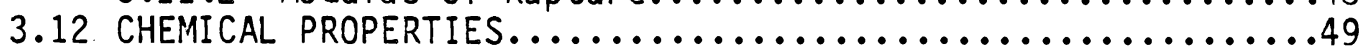

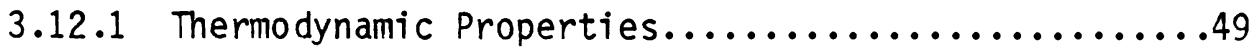

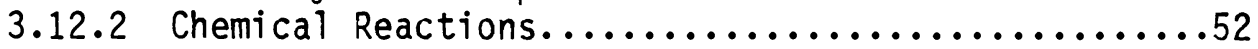

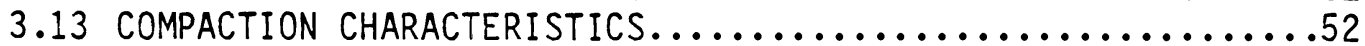

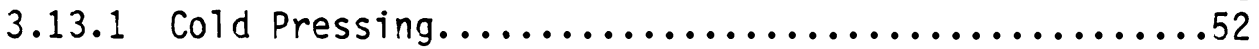




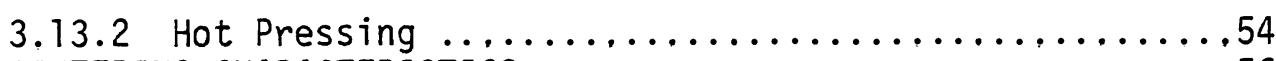

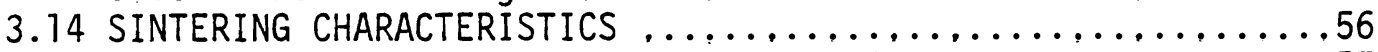

3.15 COMPATIBILITY WITH CONTAINMENT MATERIALS .............57

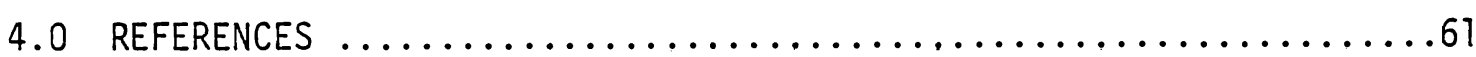




\title{
STRONTIUM-90 FLUORIDE DATA SHEET
}

\author{
H. T. Fullam
}

\subsection{INTRODUCTION}

A significant portion of the heat released by the spent fuel from nuclear reactors results from the decay of the fission product ${ }^{90} \mathrm{Sr}$ and its decay daughter 90y. Because of its relative abundance, moderately long half-life and heat output, ${ }^{90} \mathrm{Sr}$ is a candidate fuel for radioisotope-fueled power generating systems to be used in terrestrial applications. The Department of Energy (DOE) and its predecessors, the Energy Reserch and Development Administration (ERDA), and the Atomic Energy Commission (AEC), have sponsored extensive programs at the Pacific Northwest Laboratory (PNL), The Martin Company, and the Oak Ridge National Laboratory (ORNL) to develop ${ }^{90} \mathrm{Sr}$-fueled radioisotope heat sources.

The fission product strontium in spent reactor fuels consists of a mixture of stable and radioactive strontium isotopes. The isotopic composition of the strontium is influenced by several factors but depends primarily on the time since discharge of the fuel from the reactor. Typically the fission product strontium is recovered as a byproduct from the high-level fission product stream generated by reprocessing of the spent fuel using the Purex process. (1) Except for the ${ }^{90} \mathrm{Sr}$ the radioactive strontium isotopes in the fission product strontium have relatively short half-lives and will normally have decayed to low levels by the time the strontium is recovered from the reprocessing wastes. As a result, the recovered fission product strontium will usually consist of a mixture of the radioactive $90 \mathrm{Sr}$ and several stable strontium isotopes. Even though the stable isotopes normally make up $45 \%$ or more of the fission product strontium, it is typically referred to as ${ }^{90} \mathrm{Sr}$ and compounds prepared from fission product strontium are referred

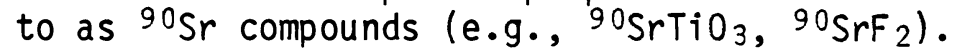

To date, terrestrial applications for $90 \mathrm{Sr}$-fueled heat sources have used strontium titanate $\left(\mathrm{SrTiO}_{3}\right)$ or strontium orthotitanate $\left(\mathrm{Sr}_{2} \mathrm{TiO}_{4}\right)$ as the fuel form (one source used $90 \mathrm{SrO}$ as the fuel form). For most applications the $90 \mathrm{Sr}$ was recovered at Hanford from a feed generated during the reprocessing of production reactor or dual purpose reactor fuels. Up to the present time no significant quantities of strontium have been recovered from commercial power reactor fuels in the United States. The strontium recovered at Hanford was shipped to the Oak Ridge National Laboratory (ORNL) or the Martin Company for conversion to the desired fuel form. ORNL and the Martin Company have prepared essentially all of the strontium-90 titanates produced in this country for heat source applications. However, production of $90 \mathrm{Sr}$ compounds by the Martin Company was halted several years ago.

Because of fission product management considerations, all of the strontium in the stored high-level wastes at Hanford is being recovered from the wastes and converted to strontium fluoride. The strontium fluoride is 
doubly encapsulated and stored in water-filled basins on the Hanford Reservation. Strontium recovery takes place in B-Plant, while the fluoride preparation, encapsulation and storage take place in the Waste Encapsulation and Storage Facilities (WESF). Both facilities were initially operated by the Atlantic Richfield Hanford Company (ARHCO) and are currently operated for the Department of Energy by Rockwell International (RHO).

Once the strontium in the Hanford wastes is converted to SrF 2 ORNL will no longer have a source of ${ }^{90} \mathrm{Sr}$ for production of the strontium titanates. ORNL has already stopped routine production of the titanates, and the equipment used for their preparation has been largely dismantled. The quantities of the two titanates available from ORNL have been reduced to negligible levels. Therefore, the only major domestic source of ${ }^{90} \mathrm{Sr}$ available for use in heat source and other potential applications is the encapsulated ${ }^{90} \mathrm{SrF}_{2}$ stored at WESF.

The use of ${ }^{9}{ }^{0} \mathrm{SrF}_{2}$ as a radioisotope heat source fuel will require qualification testing and licensing of the ${ }^{90} \mathrm{SrF}_{2}$ fuel form. The Strontium Heat Source Development Program, currently funded through the Beneficial Uses Program of the Department of Energy, has been underway at PNL for several years to develop the technology needed to permit licensing of ${ }^{90} \mathrm{SrF}_{2}$-fueled heat sources. The objective of this document is to compile in a single source al1 pertinent data developed at PNL and elsewhere on ${ }^{90} \mathrm{SrF}_{2}$ to assist potential users in developing and licensing ${ }^{9}{ }^{0} \mathrm{SrF}_{2}$-fueled heat sources for power conversion and thermal applications. Detailed data on the molecular structure, electronic structure, optical properties, etc., of ${ }^{9} \mathrm{SrF}_{2}$, which are not pertinent to, its use as a heat source fuel, are not included in the data sheet.

The data sheet is an updating of the Strontium-90 Fluoride Data Sheet (2) originally issued in 1977, which, in turn, was an updating of one section of the Strontium-90 Data Sheets prepared by S. J. Rimshaw and E. E. Ketchen of ORNL (3). The presentation of the data follows the format for other radionuclide data sheets recommended by the former AEC-Division of Isotopes Development. However, the scope has been expanded to include information on the preparation of ${ }^{9}{ }^{\circ} \mathrm{SF}_{2}$. Since WESF is the only available domestic source of ${ }^{90} \mathrm{SrF}_{2}$ much of the data presented in the report is for WESF-produced ${ }^{9} \mathrm{SrF}_{2}$ or nonradioactive $\mathrm{SrF}_{2}$ containing similar impurities. 


\subsection{PREPARATION OF ${ }^{90} \mathrm{SrF}_{2}$}

Strontium fluoride is most easily prepared by precipitation from aqueous solution. The addition of fluoride to an aqueous solution containing $0.5 \mathrm{M}$ strontium ion will precipitate in excess of $99 \%$ of the strontium as strontium fluoride.

$$
S r^{+2}+2 F^{-}=\operatorname{SrF}_{2}(s)
$$

The fluoride can be added as a soluble solid (e.g., NaF) or fluoride ion in solution. The $\mathrm{pH}$ of the solution can be varied over a fairly wide range without affecting the yield of $\mathrm{SrF}_{2}$ to any marked degree. However, an initial $\mathrm{pH}$ of 8-10 is preferred since many impurities will precipitate in this $\mathrm{pH}$ range and can be removed by filtration or settling before the SrF 2 is precipitated.

The wet precipitate can be dried by heating without significant hydrolysis. The $\mathrm{SrF}_{2}$ must be heated to at least $800^{\circ} \mathrm{C}$ to remove residual traces of moisture. Strontium fluoride fired at $1100^{\circ} \mathrm{C}$ in air or an inert atmosphere for several hours normally contains less than $50 \mathrm{ppm}$ of oxygen as water or oxide. The strontium fluoride is not hygroscopic.

\subsection{WESF FLOWSHEET FOR THE PREPARATION OF ${ }^{9} \mathrm{SrF}_{2}$}

At Hanford spent fuel from the reactors is reprocessed using the Purex process. The ${ }^{90} \mathrm{Sr}$ and other fission products from the spent fuel exit the Purex process in the high-level acjd waste stream which is neutralied and stored in tanks. The simplified flowsheet for recovery of the strontium from the fuel reprocessing waste and its purification is shown in Figure 1 . The flowsheet for conversion of the strontium to strontium fluoride and subsequent encapsulation of the $\mathrm{SrF}_{2}$ is shown in Figure 2. The strontium recovery and purification take place in B-Plant, while the fluoride conversion and encapsulation take place in the Waste Encapsulation and Storage Facilities (WESF). Both facilities are operated by Rockwell International.

In the ${ }^{9}{ }^{0} \mathrm{SrF}_{2}$ process the by-product strontium is recovered from current Purex acid waste (CAW) or Purex acid sludge (PAS) by solvent extraction. The extractant used is di-2-ethylhexylphosphoric acid (HDEHP) and tributyl phosphate (TBP) in normal paraffin hydrocarbon (NPH). The strontium is stripped from the organic phase using dilute nitric acid and purified using a series of sulfate and hydroxide precipitation steps. The purified strontium is stored in a dilute nitric acid solution until it is converted to $\mathrm{SrF}_{2}$.

A11 steps in the fluoride conversion and encapsulation process are batch operations. A volume of aqueous feed solution containing $3-5 \mathrm{~kg}$ of 


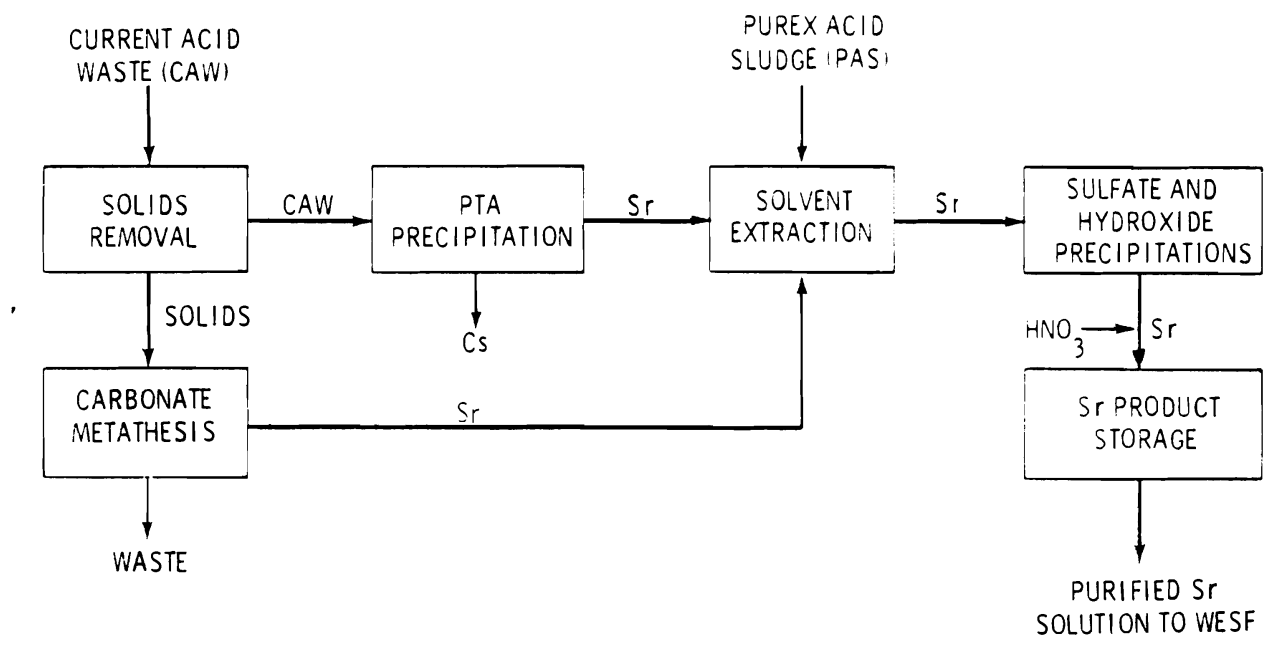

Figure 1. Simplified Flowsheet for Strontium Recovery and Purification

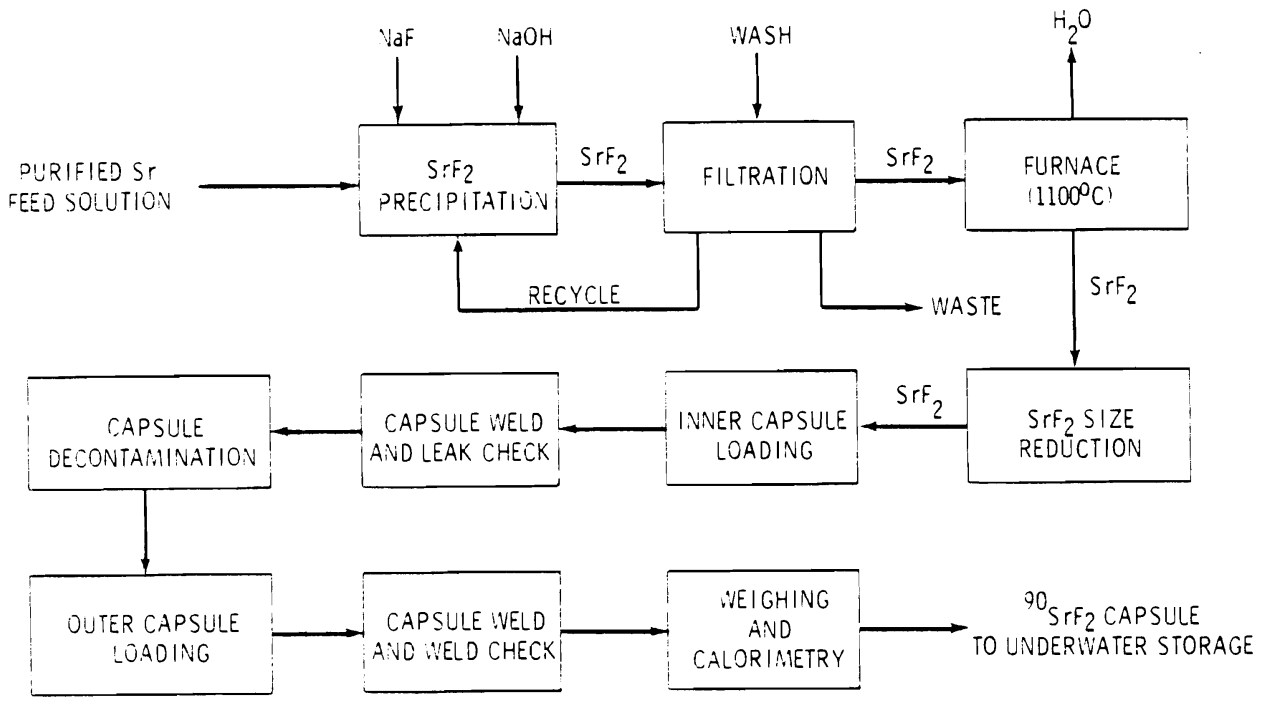

Figure 2. WESF Flowsheet for Preparation and Encapsulation of ${ }^{\circ} \mathrm{SrF}_{2}$ 
strontium is neutralized to $\mathrm{pH} 8-9$ with sodium hydroxide solution. Solid sodium fluoride is added to the solution to preciptate $\mathrm{SrF}_{2}$. The resulting slurry is digested at approximately $80^{\circ} \mathrm{C}$ for one hour with air sparging and is then filtered. The filter cake is washed with water and fired at approximately $1100^{\circ} \mathrm{C}$ in argon for several hours. After cooling, the $\mathrm{SrF}_{2}$ is pulverized to minus $1 / 2$ in. granules and loaded into a Hastelloy Alloy C-276 capsule by impact consolidation, which is essentially a cold step-pressing operation. The capsule is closed by tungsten inert gas (TIG) welding a lid in place; it is leak checked and decontaminated. The cleaned capsule is sealed in an outer capsule of $316 \mathrm{~L}$ stainless steel by TIG welding. The weld integrity of the outer capsule is checked using ultra-sonic techniques. After weighing and calorimetry to determine the heat output, the capsule is stored in a water-filled basin.

\subsection{WESF ${ }^{9} \mathrm{SrF}_{2}$ STORAGE CAPSULE}

At the present time the encapsulated ${ }^{9}{ }^{0} \mathrm{SrF}_{2}$ stored at WESF represents the only major domestic source of ${ }^{9} \mathrm{Sr}$. The ${ }^{90} \mathrm{Sr}$ isotopic content of the $\mathrm{SrF}_{2}$ current1y produced at WESF can vary from approximately $55 \%$ to as $10 \mathrm{~W}$ as $25 \%-30 \%$ depending on the age of the strontium. Pertinent data for a typical WESF storage capsule containing $\mathrm{SrF}_{2}$ having an initial ${ }^{90} \mathrm{Sr}$ isotopic content of $55 \%$ are given in Figure 3 . The heat output, power density and activity of the capsules will vary from the nominal values 1 isted depending on the initial ${ }^{90} \mathrm{Sr}$ content and will decrease with time as the ${ }^{90} \mathrm{Sr}$ decays. 


\section{STRONTIUM FLUORIDE}

1. AMOUNT $\sim 2.8 \mathrm{~kg} /$ CAPSULE

2. CHEMICAL PURITY 95\%

3. ${ }^{\circ} \mathrm{Sr}$ ISOTOPIC CONTENT $\sim 55 \%$

4. BULK DENSITY $70 \%$ OF THEORETICAL DENSITY

5. ACTIVITY $\sim 140,000 \mathrm{Ci} /$ CAPSULE

6. HEAT LOAD 960 W/CAPSULE

7. POWER DENSITY $\sim 1.0 \mathrm{w} / \mathrm{cm}^{3}$

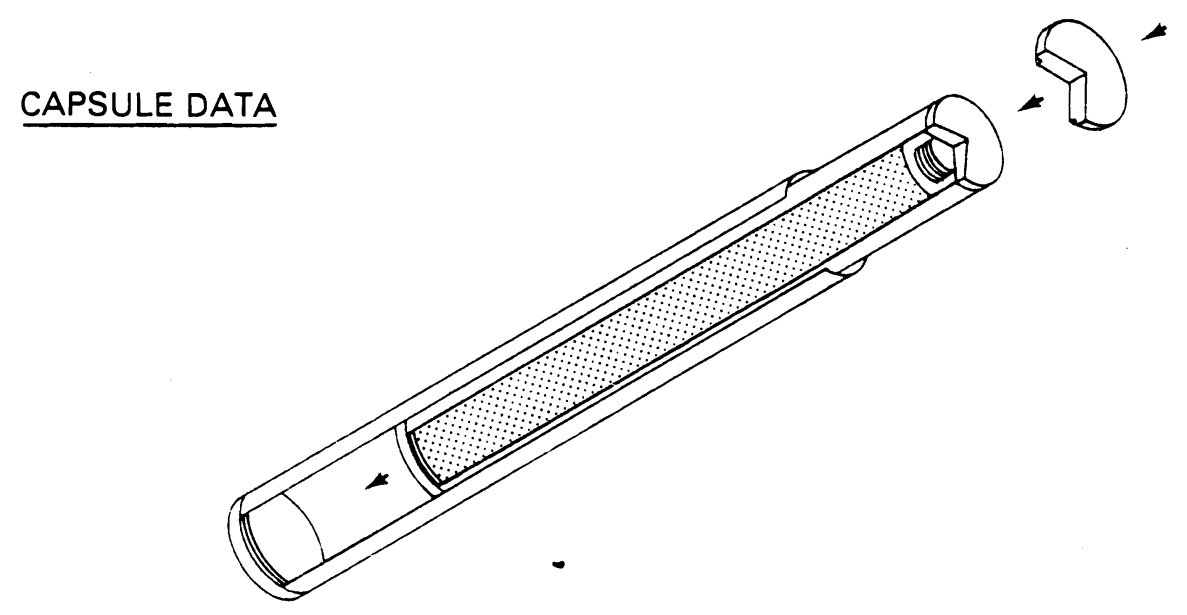

\begin{tabular}{|c|c|c|}
\hline & INNER CAPSULE & OUTER CAPSULE \\
\hline 1. MATERIAL & HASTELLOY C-276 & $316 \mathrm{~L}$ STAINLESS STEEL (a) \\
\hline 2. INNER DIAMETER & $2.01^{\prime \prime}$ & $2.39^{\prime \prime}$ \\
\hline 3. OUTER DIAMETER & $2.25^{\prime \prime}$ & $2.63^{\prime \prime}$ \\
\hline 4. TOTAL LENGTH & $19.05^{\prime \prime}$ & $20.10^{\prime \prime}$ \\
\hline
\end{tabular}

(a) HASTELLOY C-276 WAS USED FOR THE OUTER CAPSULE DURING THE EARLY STAGES OF THE WESF OPERATION

Figure 3. Typical Data (At Time of Capsule Loading) for a WESF 'SSrF, Storage Capsule 


\subsection{PROPERTIES OF STRONTIUM FLUORIDE}

Data that have been reported in the literature on the properties of strontium fluoride are summarized in the following sections. Some of the data were obtained using ${ }^{9}{ }^{0} \mathrm{SrF}_{2}$, but most were obtained using nonradioactive $\mathrm{SrF}_{2}$. The data obtained with ${ }^{\circ} \mathrm{SrF}_{2}$ are so indicated. Some properties of $\mathrm{SrF}_{2}$ were calculated using theoretical techniques or estimated from data on similar compounds. The calculated or estimated values are indicated as such.

The quality of the $\mathrm{SrF}_{2}$ used in making the property measurements is included when available. In the PNL studies three grades of nonradioactive $\mathrm{SrF}_{2}$ were used for most property measurements. They were:

- Pure $\mathrm{SrF}_{2}(<0.1 \%$ total impurities)

- Commercial $\mathrm{SrF}_{2}$ (0.5\% total impurities)

- WESF-Grade $\mathrm{SrF}_{2}$ ( $25 \%$ impurities similar to those found in WESF-produced ${ }^{9} \mathrm{SrF}_{2}$ ).

WESF-produced ${ }^{9} \mathrm{SrF}_{2}$, or ${ }^{9}{ }^{0} \mathrm{SrF}_{2}$ produced at PNL using the WESF flowsheet and ${ }^{\circ} \mathrm{Sr}$ solution from ARHCO, was used for property measurements where the radioactivity was expected to affect the results obtained (i.e., dissolution rate, compatibility).

\subsection{HALF-LIFE}

$$
\begin{aligned}
& { }^{90} \mathrm{Sr}-28.6 \text { years }(4) \\
& { }^{89} \mathrm{Sr}-50.5 \text { days }(5) \\
& { }^{9} \mathrm{Sr}-9.5 \text { hours }(5) \\
& { }^{90} \mathrm{Y}-64.1 \text { hours }(4)
\end{aligned}
$$

\subsection{COMPOSITION}

\subsubsection{Isotopic Composition}

The isotopic composition of fission product strontium is influenced by a number of factors but will depend primarily on the time since the strontium was discharged from the reactor. Unintentional additions of natural strontium during fuel reprocessing and strontium recovery will also have some effect on the isotopic com- 
position. Because of its relatively short half-life, any ${ }^{91} \mathrm{Sr}$ initially present in the discharged fuel will have decayed to a negligible level by the time the strontium is recovered from the waste. The calculated activity and atomic ratios for ${ }^{89} \mathrm{Sr}-{ }^{90} \mathrm{Sr}$ as a function of time since reactor discharge for an enriched uranium power reactor fuel are shown below. The ${ }^{89} \mathrm{Sr} /{ }^{90} \mathrm{Sr}$ ratio decreases by a factor of 2 with every $503 / 4$ days of cooling.

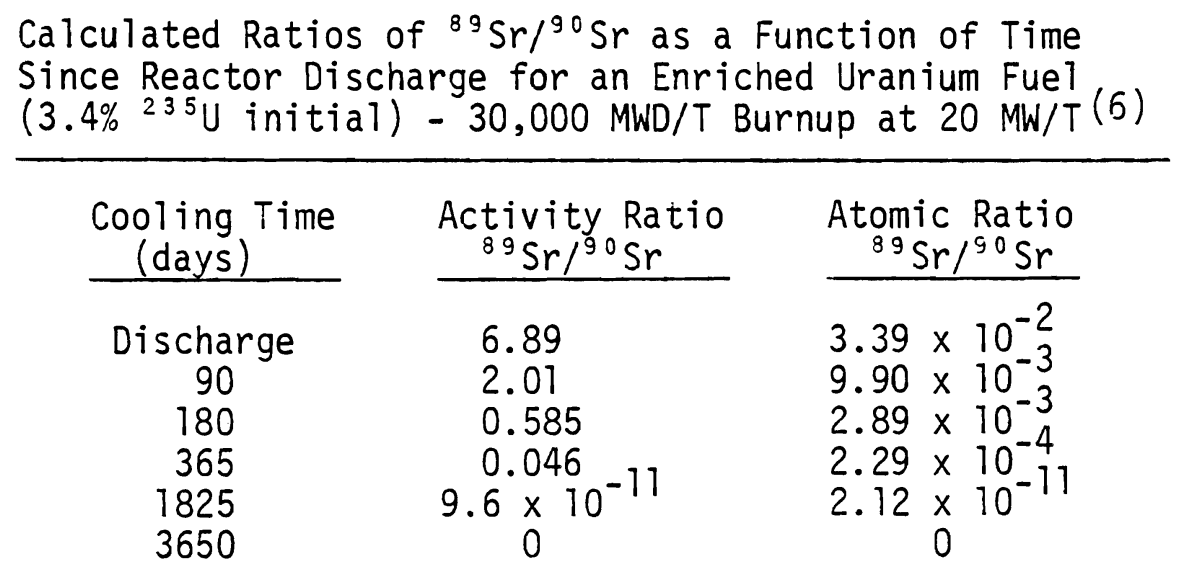

A typical analysis of fission product strontium recovered from Hanford wastes is shown below. The waste had aged sufficiently at the time of strontium recovery that the ${ }^{89} \mathrm{Sr}$ had decayed to a nondetectable level. A part of the stable strontium isotopes resulted from unintentional additions of small amounts of natural strontium with various chemical streams during the processing operations.

Typical Isotopic Analysis of Fission Product Strontium Recovered From Hanford Wastes (7)

\begin{tabular}{lc}
\hline Isotope & Atomic $\%$ \\
\cline { 2 - 2 } $90 \mathrm{Sr}$ & 55.10 \\
$89 \mathrm{Sr}$ & 43.80 \\
$87 \mathrm{Sr}$ & 0.40 \\
$85 \mathrm{Sr}$ & 0.57 \\
$3 \mathrm{Sr}$ & 0.10 \\
\hline
\end{tabular}




\subsubsection{Radiochemical Composition}

The radiochemical purity of fission product strontium will depend primarily on the time since discharge from the reactor and on the effectiveness of the purification procedures used in strontium recovery. The principal radiochemical impurities which may be present in fission product strontium include the following:

\begin{tabular}{|c|c|c|c|}
\hline Isotope & $t_{1},(5)$ & Isotope & $t_{1} \frac{(5)}{3}$ \\
\hline $91 Y$ & $58.6 \mathrm{~d}$ & $13{ }^{4} \mathrm{Cs}$ & 2.06 \\
\hline${ }^{9} \mathrm{Zr}$ & $65.5 \mathrm{~d}$ & $137 \mathrm{Cs}$ & $30.1 \mathrm{y}$ \\
\hline${ }^{95} \mathrm{Nb}$ & $35.1 \mathrm{~d}$ & $13^{7} \mathrm{mBa}$ & $2.55^{\circ}$ \\
\hline $106 \mathrm{Ru}$ & $369 d$ & $1{ }^{44} \mathrm{Ce}$ & $284 \mathrm{~d}$ \\
\hline $106 \mathrm{Rh}$ & $2.18 \mathrm{~h}$ & $144 \mathrm{Pr}$ & $17.3 \mathrm{~m}$ \\
\hline
\end{tabular}

Strontium recovered from Hanford wastes has been reported to contain up to $0.03 \%$ of the total activity as ${ }^{144} \mathrm{Ce}$ and $0.003 \%$ as ${ }^{137} \mathrm{Cs}$. Analysis of strontium fluoride produced at WESF shows the only measurable activity other than ${ }^{90} \mathrm{Sr}-{ }^{90} \mathrm{Y}$ is ${ }^{13}{ }^{7} \mathrm{CS}$. WESF ${ }^{90} \mathrm{SrF}_{2}$, which was about 18 months old at the time of analysis, contained approximately $2.8 \times 10^{-5} \mathrm{Ci}$ of ${ }^{137} \mathrm{Cs}$ per $\mathrm{Ci}$ of ${ }^{90} \mathrm{Sr}$. (8)

The WESF-produced $\mathrm{SrF}_{2}$ will contain ${ }^{90} \mathrm{Y}$ which is the decay daughter of $90 \mathrm{Sr}$. Once secular equjlibrium is established, the ${ }^{9} \mathrm{Y} /{ }^{90} \mathrm{Sr}$ atomic ratio is $2.56 \times 10^{-4}$.

\subsubsection{Chemical Composition}

The strontium fluoride produced at WESF will contain substantial quantities of cation impurities and much lesser amounts of anion impurities. The impurity concentrations can vary over a considerable range. Strontium recovered from different waste tanks will, in general, contain the same kinds of impurities, but the concentrations may vary. Since the procedures for purifying the strontium are only partially effective, the impurity concentrations of the ${ }^{90} \mathrm{SrF}_{2}$ product will vary with the source of the strontium. Corrosion of process equipment, especially in the fluoride firing operation, will also contribute to the impurities in the WESF ${ }^{90} \mathrm{SrF}_{2}$. Some product nonhomogeneity will result from the corrosion of process equipment. The impurities normally found in WESF-produced ${ }^{9}{ }^{\circ} \mathrm{SrF}_{2}$ and their probable concentration ranges are presented below: 
Typical Impurities Found in WESF-Produced ${ }^{90} \mathrm{SrF}_{2}$ and Their Probable Concentration Ranges(7)

\begin{tabular}{|c|c|c|c|}
\hline Component & $w t \%$ & Component & wt\% \\
\hline $\begin{array}{l}\text { Sr } \\
F\end{array}$ & $\begin{array}{l}66 \text { (nominal) } \\
30 \text { (nominal) }\end{array}$ & $\begin{array}{l}\mathrm{Mn} \\
\mathrm{Na} \\
\mathrm{Ni}\end{array}$ & $\begin{array}{r}<0.1 \\
1-4 \\
<0.1\end{array}$ \\
\hline $\begin{array}{l}\mathrm{Al} \\
\mathrm{Ba} \\
\mathrm{Ca} \\
\mathrm{Cd} \\
\mathrm{Cr} \\
\mathrm{Cu} \\
\mathrm{Fe} \\
\mathrm{K} \\
\mathrm{Mg}\end{array}$ & $\begin{array}{c}<0.5 \\
0.1-2.0 \\
0.1-2.0 \\
<0.1 \\
<0.2 \\
<0.01 \\
<0.1 \\
<0.01 \\
0.05-0.5\end{array}$ & $\begin{array}{l}\mathrm{Pb} \\
\mathrm{RE}(\mathrm{a}) \\
\mathrm{Si} \\
\mathrm{Zr}(\mathrm{b}) \\
\mathrm{H} \\
\mathrm{O} \\
\mathrm{NO}_{3}^{-} \\
\mathrm{SO}_{4}^{-2}\end{array}$ & $\begin{array}{c}<0.2 \\
<2.0 \\
<0.02 \\
\text { Variable } \\
<0.01 \\
<0.05 \\
<0.05 \\
<0.01\end{array}$ \\
\hline \multicolumn{4}{|c|}{ (a) RE - Rare Earth Elements } \\
\hline
\end{tabular}

The strontium fluoride produced at WESF usually contains about 94 wt\% strontium fluoride and 6 wt\% impurities, principally metal fluorides. A typical batch analysis is given in the following table.

Typical Batch Analysis of WESF Produced Strontium-90 Fluoride

\begin{tabular}{|c|c|c|c|}
\hline Element & $w t \%$ & Element & $\mathrm{Wt} \%$ \\
\hline $\begin{array}{r}\mathrm{Sr} \\
\mathrm{Al} \\
-\mathrm{Ba} \\
\mathrm{Ca} \\
\mathrm{Cd} \\
\mathrm{Cr} \\
\mathrm{Fe}\end{array}$ & $\begin{array}{l}65.6 \\
0.22 \\
0.59 \\
0.39 \\
0.02 \\
0.32 \\
0.41\end{array}$ & $\begin{array}{l}\mathrm{Mg} \\
\mathrm{Mn} \\
-\mathrm{Na} \\
\mathrm{Ni} \\
\mathrm{Pb} \\
\mathrm{RE}(\mathrm{a}) \\
\mathrm{Zr}(\mathrm{b})\end{array}$ & $\begin{array}{l}0.03 \\
0.01 \\
1.9 \\
0.26 \\
0.01 \\
1.1 \\
0.8\end{array}$ \\
\hline
\end{tabular}

(a) RE - Rare earth elements

(b) Decay product - will increase as the ${ }^{90} \mathrm{Sr}$ decays 


\subsection{DECAY SCHEME} 90 Sr Decay ${ }^{(4)}$

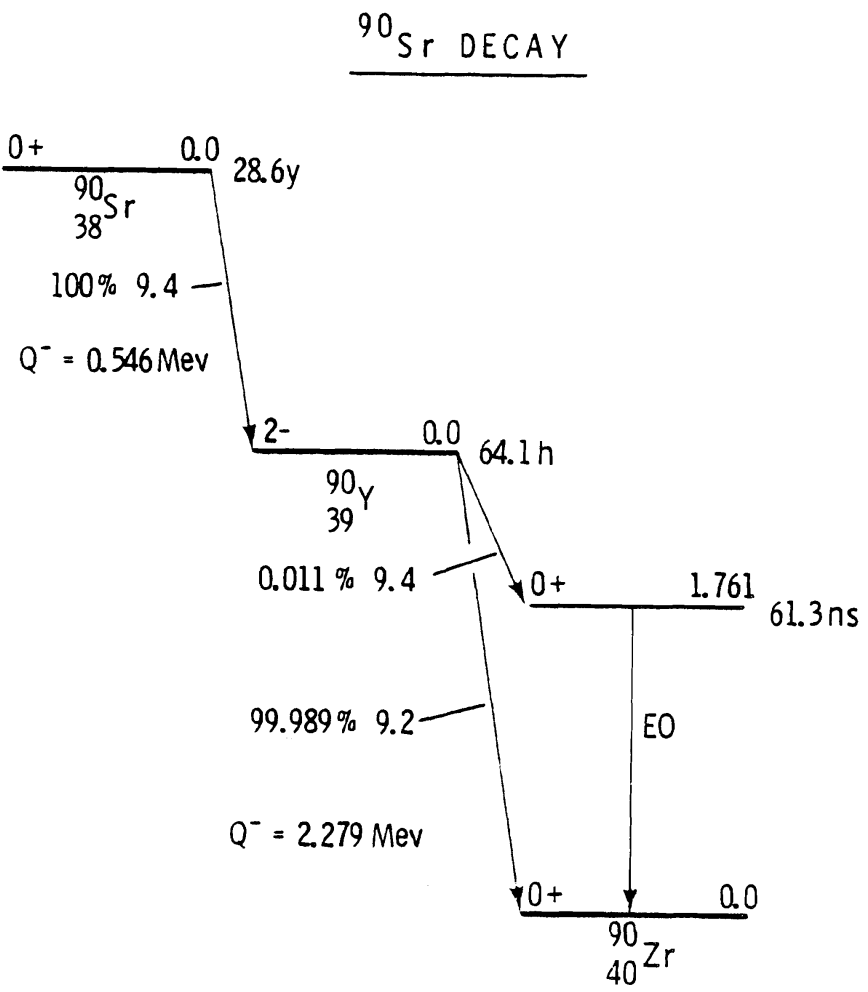

The chemical species resulting from the decay of the ${ }^{90} \mathrm{Sr}$ in $\mathrm{SrF}_{2}$ have not been identified experimentally. The ${ }^{\circ}{ }^{\circ} \mathrm{SrF}_{2}$ should decay to the difluoride of stable ${ }^{90} \mathrm{Zr}$.

$$
{ }^{90} \mathrm{SrF}_{2} \rightarrow\left[{ }^{90} \mathrm{YF}_{2}\right] \rightarrow{ }^{90} \mathrm{ZrF}_{2}
$$

Zirconium difluoride has been reported in the literature(9) but is said to disproportionate at elevated temperatures to zirconium tetrafluoride and zirconium metal powder.

$$
2 \mathrm{ZrF}_{2} \rightarrow \mathrm{ZrF}_{4}+\mathrm{Zr}
$$

Any $\mathrm{ZrF}_{2}$ formed as a decay product in a ${ }^{90} \mathrm{SrF}_{2}$ heat source should disproportionate at source operating temperatures, but this has not been demonstrated experimentally. The possibility also exists that the $\mathrm{ZrF}_{2}$ 'could react with the $\mathrm{SrF}_{2}$, or impurity fluorides present, to form complex fluorides. 
${ }^{89} \mathrm{Sr}$ Decay $(10)$

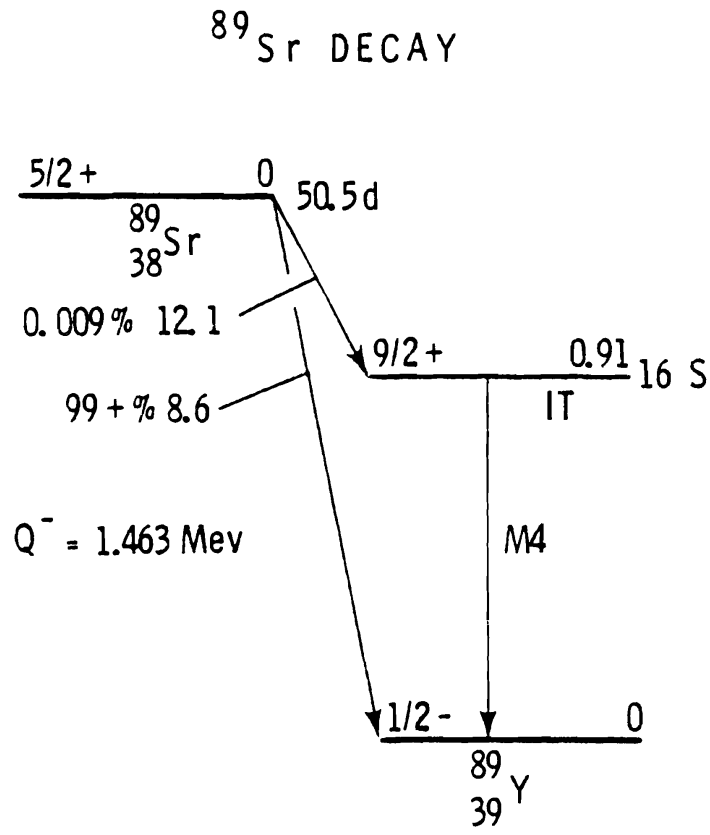

\subsection{SPECIFIC ACTIVITY $(11,12)$}

a. ${ }^{90} \mathrm{Sr}(100 \%$ Basis $)$

$139 \mathrm{Ci} / \mathrm{g}$

$149 \mathrm{Ci} / \mathrm{W}(\mathrm{t})$

$0.933 W(t) / g$

b. Pure $\mathrm{SrF}_{2}\left(70.1 \% \mathrm{Sr}, 55 \%{ }^{90} \mathrm{Sr}\right.$ Isotopic Content)

$53.6 \mathrm{Ci} / \mathrm{g}$

$0.360 w(t) / g$

c. WESF $\mathrm{SrF}_{2}\left(95 \% \mathrm{SrF}_{2}, 55 \%{ }^{90} \mathrm{Sr}\right.$ Isotopic Content)

$50.9 \mathrm{Ci} / \mathrm{g}$

$0.342 W(t) / g$

$1.02 W(t) / \mathrm{cm}^{3}$ (at $70 \%$ of theoretical density)

The heat output of the ${ }^{90} \mathrm{SrF}_{2}$ will decrease with time as the ${ }^{90} \mathrm{Sr}$ decays.

The following table shows this relationship for WESF ${ }^{90} \mathrm{SrF}_{2}$ of $95 \%$ chemical purity and having an initial ${ }^{90} \mathrm{Sr}$ isotopic content of $55 \%$. 


\begin{tabular}{|c|c|c|c|c|}
\hline \multirow[b]{2}{*}{$\begin{array}{l}\text { Time } \\
\text { yr }\end{array}$} & \multirow{2}{*}{$\begin{array}{c}\text { Sr Isotopic } \\
\text { Content } \\
\%\end{array}$} & \multirow[b]{2}{*}{$\begin{array}{c}\text { Activity } \\
\mathrm{Ci} / \mathrm{g}\end{array}$} & \multicolumn{2}{|c|}{ Heat Output } \\
\hline & & & $w(t) / g$ & $w(t) / \mathrm{cm}^{3(b)}$ \\
\hline $\begin{array}{l}0 \\
1 \\
2 \\
5 \\
10 \\
20 \\
28.6 \\
57.2\end{array}$ & $\begin{array}{l}55.0 \\
54.4 \\
53.8 \\
52.0 \\
49.0 \\
42.9 \\
37.9 \\
23.4\end{array}$ & $\begin{array}{l}50.9 \\
49.7 \\
48.5 \\
45.1 \\
39.9 \\
31.3 \\
25.4 \\
12.7\end{array}$ & $\begin{array}{l}0.342 \\
0.334 \\
0.326 \\
0.303 \\
0.268 \\
0.211 \\
0.171 \\
0.0855\end{array}$ & $\begin{array}{l}1.02 \\
0.991 \\
0.968 \\
0.899 \\
0.795 \\
0.626 \\
0.508 \\
0.254\end{array}$ \\
\hline
\end{tabular}

(a) WESF $^{9}{ }^{0} \mathrm{SrF}_{2}$ of $95 \%$ chemical purity

(b) ${ }^{9} \mathrm{SrF}_{2}$ compacted to $70 \%$ of theoretical density

\subsection{RADIATION}

a. Alpha Particles

None

b. Beta Particles $(4,12,13)$

\begin{tabular}{|c|c|c|c|c|c|}
\hline Nuclide & $\underset{\text { Mev }}{\text { Max. E }}$ & $\begin{array}{c}\text { Ave. E } \\
\text { Mev }\end{array}$ & $\begin{array}{c}\text { Abundance } \\
\%\end{array}$ & W/ kCi & $\frac{\text { PParticles }}{w-\sec }$ \\
\hline${ }^{90} \mathrm{Sr}$ & 0.546 & 0.196 & 100 & 1.16 & $5.51 \times 10^{12}$ \\
\hline \multirow[t]{2}{*}{$90 y$} & 2.279 & 0.937 & 100 & $\underline{5.55}$ & $5.51 \times 10^{12}$ \\
\hline & \multicolumn{3}{|c|}{ Total Power } & 6.71 & \\
\hline
\end{tabular}

c. Gamma

None

d. Bremsstrahlung $(12,13)$

The high-energy bremsstrahlung photons produced from ${ }^{\circ} \mathrm{Sr}$ and ${ }^{90} \mathrm{Y}$ in a $\mathrm{SrF}_{2}$ matrix have not been determined. However Arnold(12) at ORNL has calculated the bremsstrahlung photons for $\mathrm{SrO}$ and $\mathrm{SrTiO}_{3}$ matrices and the data are presented in the tables on pages 14-17. The bremsstrahlung photons from a $\mathrm{SrF}_{2}$ matrix should 7 ie between those for $\mathrm{SrO}$ and $\mathrm{SrTiO}_{3}$ but closer to Sro. 
Sources containing ${ }^{90} \mathrm{Sr}-{ }^{90} \mathrm{Y}$ activities require slightly more shielding than ${ }^{1{ }^{3}} \mathrm{Cs}-137 \mathrm{mBa}$ sources because of the high energy bremsstrahlung radiation from ${ }^{9} \mathrm{Y}$ which emits a $2.279 \mathrm{Mev}$ beta particle.

e. Neutrons

None

Production of Bremsstrahlung Photons

from Strontium-90 Beta in Strontium Oxide Matrix (12)

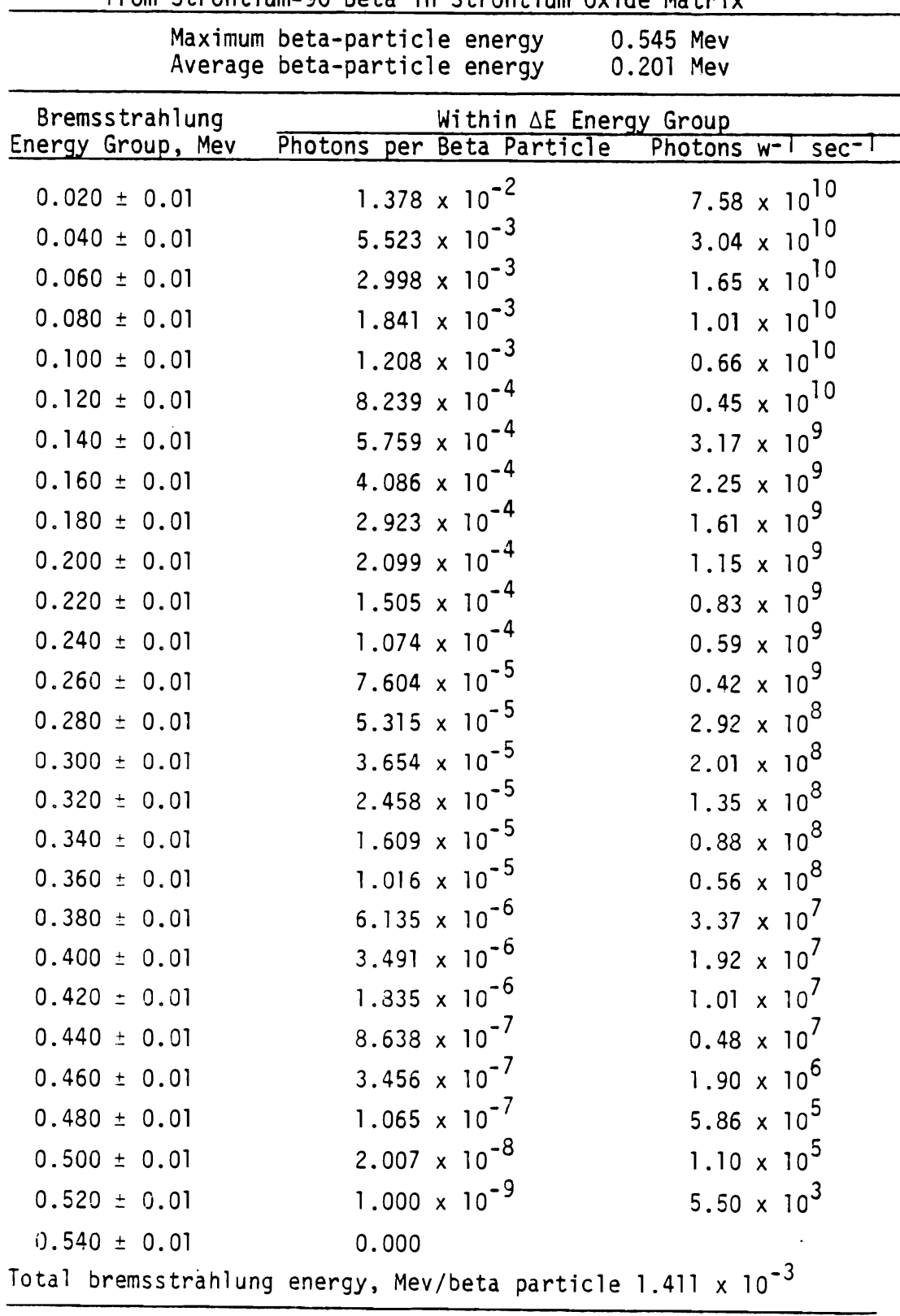


Production of Bremsstrahlung Photons from Yttrium-90 Beta in Strontium Oxide Matrix (12)
Maximum beta-particle energy
2.27 Mev
Average beta-particle energy
$0.944 \mathrm{Mev}$

Bremsstrahlung

Within $\triangle E$ Energy Group Energy Group Mev Photons per Beta Particle

Photons $\mathrm{w}^{-1} \mathrm{sec}^{-T}$

\begin{tabular}{|c|c|c|}
\hline $0.100 \pm 0.05$ & $6.152 \times 10^{-2}$ & $3.38 \times 10^{11}$ \\
\hline $0.200 \pm 0.05$ & $2.415 \times 10^{-2}$ & $1.33 \times 10^{11}$ \\
\hline $0.300 \pm 0.05$ & $1.281 \times 10^{-2}$ & $0.70 \times 10^{11}$ \\
\hline $0.400 \pm 0.05$ & $7.674 \times 10^{-3}$ & $4.22 \times 10^{10}$ \\
\hline $0.500 \pm 0.05$ & $4.894 \times 10^{-3}$ & $2.69 \times 10^{10}$ \\
\hline $0.600 \pm 0.05$ & $3.234 \times 10^{-3}$ & $1.78 \times 10^{10}$ \\
\hline $0.700 \pm 0.05$ & $2.181 \times 10^{-3}$ & $1.20 \times 10^{10}$ \\
\hline $0.800 \pm 0.05$ & $1.485 \times 10^{-3}$ & $8.17 \times 10^{9}$ \\
\hline $0.900 \pm 0.05$ & $1.014 \times 10^{-3}$ & $5.58 \times 10^{9}$ \\
\hline $1.000 \pm 0.05$ & $6.887 \times 10^{-4}$ & $3.79 \times 10^{9}$ \\
\hline $1.100 \pm 0.05$ & $4.630 \times 10^{-4}$ & $2.55 \times 10^{9}$ \\
\hline $1.200 \pm 0.05$ & $3.000 \times 10^{-4}$ & $1.65 \times 10^{9}$ \\
\hline $1.300=0.05$ & $1.973 \times 10^{-4}$ & $1.09 \times 10^{9}$ \\
\hline $1.400 \pm 0.05$ & $1.231 \times 10^{-4}$ & $0.68 \times 10^{9}$ \\
\hline $1.500 \pm 0.05$ & $7.337 \times 10^{-5}$ & $4.04 \times 10^{8}$ \\
\hline $1.600 \pm 0.05$ & $4.117 \times 10^{-5}$ & $2.26 \times 10^{8}$ \\
\hline $1.700 \pm 0.05$ & $2.125 \times 10^{-5}$ & $1.17 \times 10^{8}$ \\
\hline $1.800 \pm 0.05$ & $9.740 \times 10^{-6}$ & $5.36 \times 10^{7}$ \\
\hline $1.900 \pm 0.05$ & $3.728 \times 10^{-6}$ & $2.05 \times 10^{7}$ \\
\hline $2.000 \pm 0.05$ & $1.058 \times 10^{-6}$ & $5.82 \times 10^{6}$ \\
\hline $2.100 \pm 0.05$ & $1.670 \times 10^{-7}$ & $9.19 \times 10^{5}$ \\
\hline $2.200 \pm 0.05$ & $4.768 \times 10^{-9}$ & $2.62 \times 10^{4}$ \\
\hline
\end{tabular}


Production of Bremsstrahlung Photons from Strontium-90 Beta in Strontium Titanate Matrix (12)

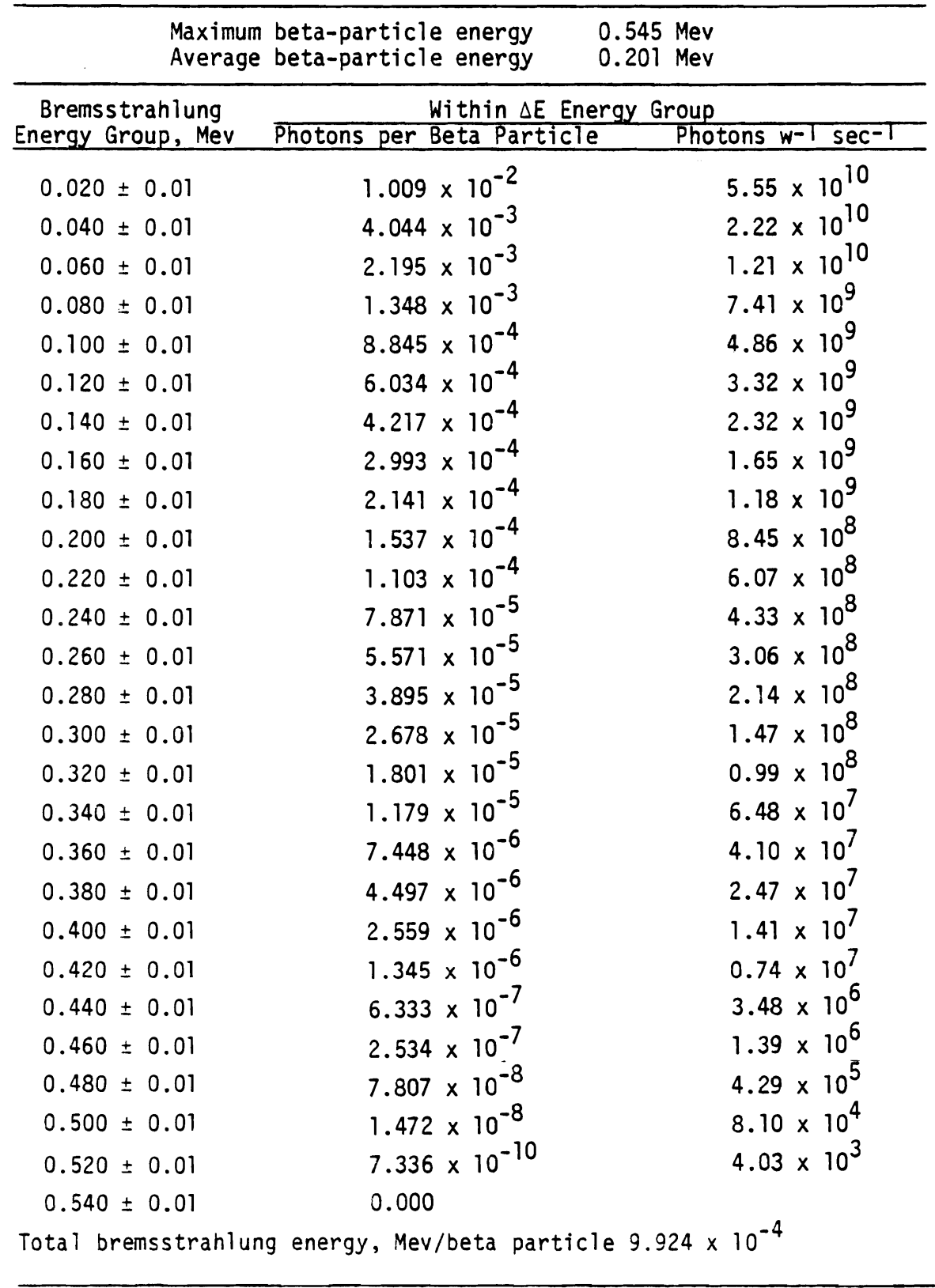


Production of Bremsstrahlung Photons from Yttrium-90 Beta in Strontium Titanate Matrix (12)

\begin{tabular}{|c|c|c|c|c|c|}
\hline \multirow{3}{*}{$\begin{array}{r}\text { Maximun } \\
\text { Average } \\
\text { Bremsstrahlung } \\
\text { Energy Group, Mev } \\
\end{array}$} & $\begin{array}{l}\text { beta-pa } \\
\text { beta-pa }\end{array}$ & $\begin{array}{l}\operatorname{articl} \\
\operatorname{articl}\end{array}$ & $\begin{array}{l}\text { le energy } \\
\text { le energy }\end{array}$ & $\begin{array}{l}.27 \mathrm{Mev} \\
.944 \mathrm{Mev}\end{array}$ & \\
\hline & \multicolumn{5}{|c|}{ Within $\Delta E$ Energy Group } \\
\hline & Photons & 5 per & Beta Particle & Photons & $w^{-1} \mathrm{sec}^{-1}$ \\
\hline $0.100 \pm 0.05$ & \multicolumn{3}{|c|}{$4.537 \times 10^{-2}$} & 2.50 & $\times 10^{11}$ \\
\hline $0.200 \pm 0.05$ & \multicolumn{3}{|c|}{$1.782 \times 10^{-2}$} & 0.98 & $\times 10^{11}$ \\
\hline $0.300 \pm 0.05$ & \multicolumn{3}{|c|}{$9.456 \times 10^{-3}$} & 5.20 & $\times 10^{10}$ \\
\hline $0.400 \pm 0.05$ & \multicolumn{3}{|c|}{$5.665 \times 10^{-3}$} & 3.12 & $\times 10^{10}$ \\
\hline $0.500 \pm 0.05$ & \multicolumn{3}{|c|}{$3.613 \times 10^{-3}$} & 1.99 & $\times 10^{10}$ \\
\hline $0.600 \div 0.05$ & \multicolumn{3}{|c|}{$2.389 \times 10^{-3}$} & 1.31 & $\times 10^{10}$ \\
\hline $0.700 \pm 0.05$ & \multicolumn{3}{|c|}{$1.611 \times 10^{-3}$} & 0.89 & $\times 10^{10}$ \\
\hline $0.800 \pm 0.05$ & \multicolumn{3}{|c|}{$1.098 \times 10^{-3}$} & 6.04 & $\times 10^{9}$ \\
\hline $0.900 \pm 0.05$ & \multicolumn{3}{|c|}{$7.493 \times 10^{-4}$} & 4.12 & $\times 10^{9}$ \\
\hline $1.000 \pm 0.05$ & \multicolumn{3}{|c|}{$5.092 \times 10^{-4}$} & 2.80 & $\times 10^{9}$ \\
\hline $1.100 \pm 0.05$ & \multicolumn{3}{|c|}{$3.425 \times 10^{-4}$} & 1.88 & $\times 10^{9}$ \\
\hline $1.200 \pm 0.05$ & \multicolumn{3}{|c|}{$2.264 \times 10^{-4}$} & 1.25 & $\times 10^{9}$ \\
\hline $1.300 \pm 0.05$ & \multicolumn{3}{|c|}{$1.460 \times 10^{-4}$} & 8.03 & $\times 10^{8}$ \\
\hline $1.400 \pm 0.05$ & \multicolumn{3}{|c|}{$9.110 \times 10^{-5}$} & 5.01 & $\times 10^{8}$ \\
\hline $1.500 \pm 0.05$ & \multicolumn{3}{|c|}{$5.434 \times 10^{-5}$} & 3.00 & $\times 10^{8}$ \\
\hline $1.600 \pm 0.05$ & \multicolumn{3}{|c|}{$3.050 \times 10^{-5}$} & 1.68 & $\times 10^{8}$ \\
\hline $1.700=0.05$ & \multicolumn{3}{|c|}{$1.576 \times 10^{-5}$} & 8.67 & $\times 10^{7}$ \\
\hline $1.800 \pm 0.05$ & \multicolumn{3}{|c|}{$7.220 \times 10^{-6}$} & 3.97 & $\times 10^{7}$ \\
\hline $1.900 \pm 0.05$ & \multicolumn{3}{|c|}{$2.764 \times 10^{-6}$} & 1.52 & $\times 10^{7}$ \\
\hline $2.000 \pm 0.05$ & \multicolumn{3}{|c|}{$7.850 \times 10^{-7}$} & 4.32 & $\times 10^{6}$ \\
\hline $2.100 \pm 0.05$ & \multicolumn{3}{|c|}{$1.239 \times 10^{-7}$} & 6.81 & $\times 10^{5}$ \\
\hline $2.200 \pm 0.05$ & \multicolumn{3}{|c|}{$3.540 \times 10^{-9}$} & 1.95 & $\times 10^{4}$ \\
\hline \multicolumn{4}{|c|}{ Total bremsstrahlung energy, Mev/beta particl } & 1e 2.078 & $\times 10^{-2}$ \\
\hline
\end{tabular}




\subsection{SHIELDING DATA $(12)$}

Shielding calculations for ${ }^{9} \mathrm{SrF}_{2}$ sources have not been carried out. Arnold $\left(1_{2}\right)$ at ORNL has calculated the bremsstrahlung dose rates for ${ }^{90} \mathrm{Sr}$ sources having $\mathrm{SrO}$ and $\mathrm{SrTiO}_{3}$ matrices. The calculated dose rates for unshielded and shielded sources of various sizes are presented in Figures 4 - 11. Shielding requirements for ${ }^{\circ} \mathrm{SrF}_{2}$ sources are expected to be intermediate between those of equivalent sized ${ }^{90}$ Sro and ${ }^{\circ} \mathrm{SrTiO}_{3}$ sources, but closer to those of ${ }^{9}{ }^{\circ} \mathrm{SrO}$. For example, Arnold's calculations show that 500 watt sources of ${ }^{9}{ }^{0} \mathrm{SrO}$ and ${ }^{9}{ }^{0} \mathrm{SrTiO}_{3}$ would require about $14 \frac{1}{2} \mathrm{~cm}$. and $13 \frac{1}{2} \mathrm{~cm}$. of lead shielding respectively to reduce the dose rate to $10 \mathrm{millirad} / \mathrm{hr}$ at a distance of $100 \mathrm{~cm}$. A ${ }^{90}{ }^{0} \mathrm{SrF}_{2}$ source of the same size would probably require between $14-14 \frac{1}{2} \mathrm{~cm}$. of lead shielding to attain the same dose rate. The ${ }^{90} \mathrm{Sr}$ sources require substantial shielding because of the high-energy bremsstrahlung radiation resulting from 90 y decay.

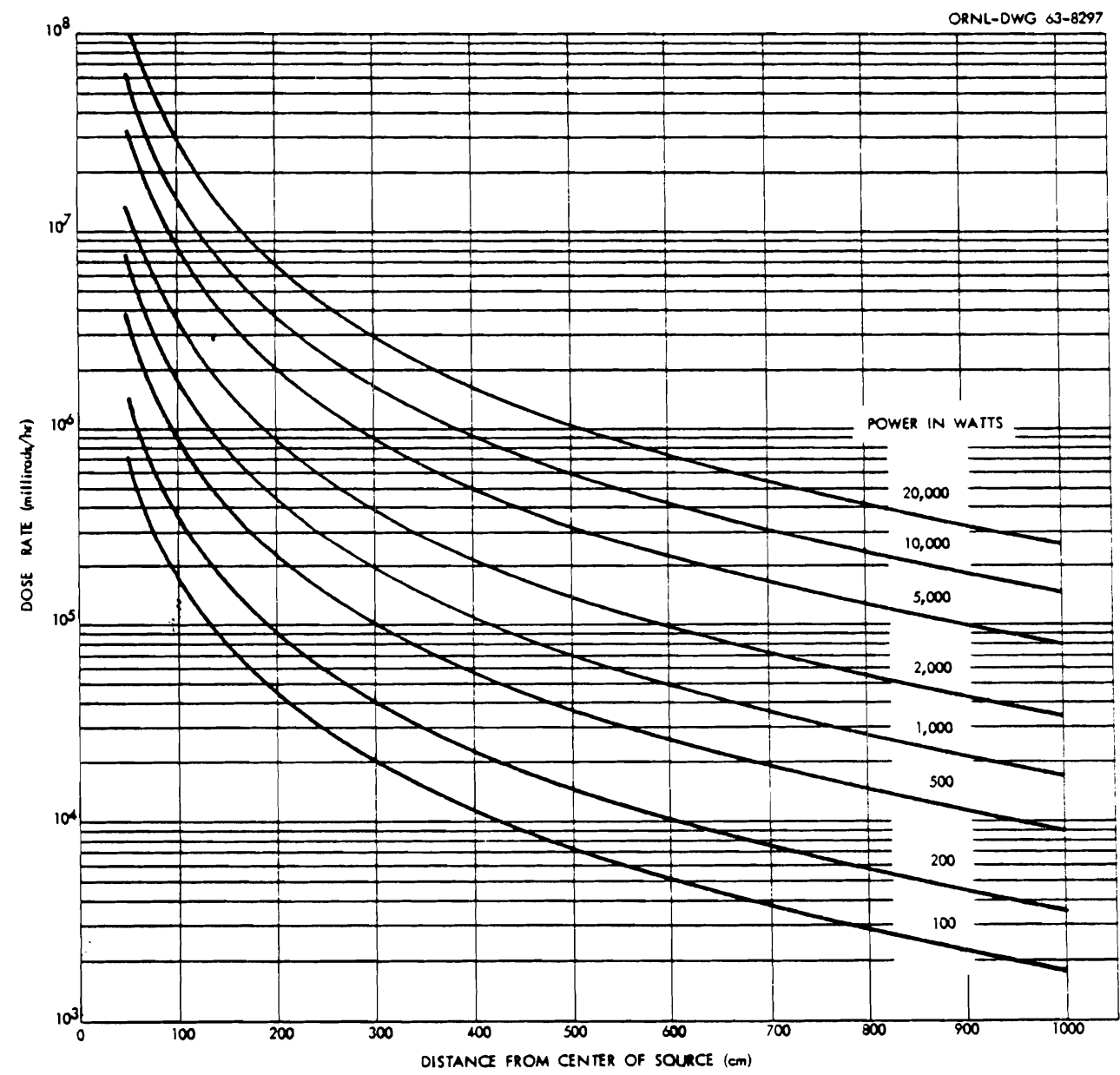

Figure 4. Bremsstrahlung Dose Rates from Unshielded Isotopic Power Sources of Strontium-90 (Strontium 0xide) as a Function of Distance from Center of Source. (12) 


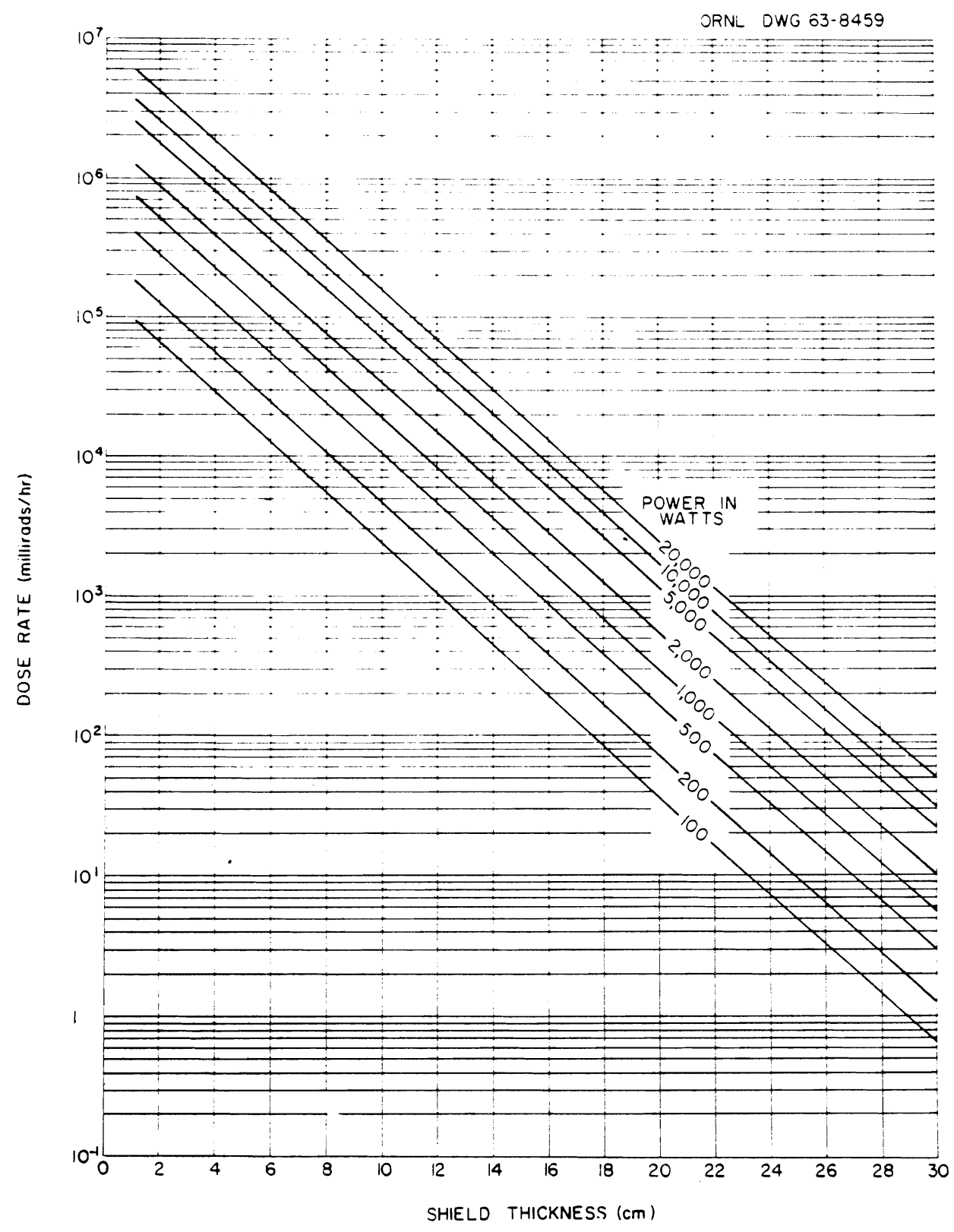

Figure 5. Bremsstrahlung Dose Rates from Iron-Shielded Isotopic Power Sources of Strontium-90 (Strontium Oxide). Genfer of source to dose point separation distance $=100 \mathrm{~cm}$. $(12)$ 


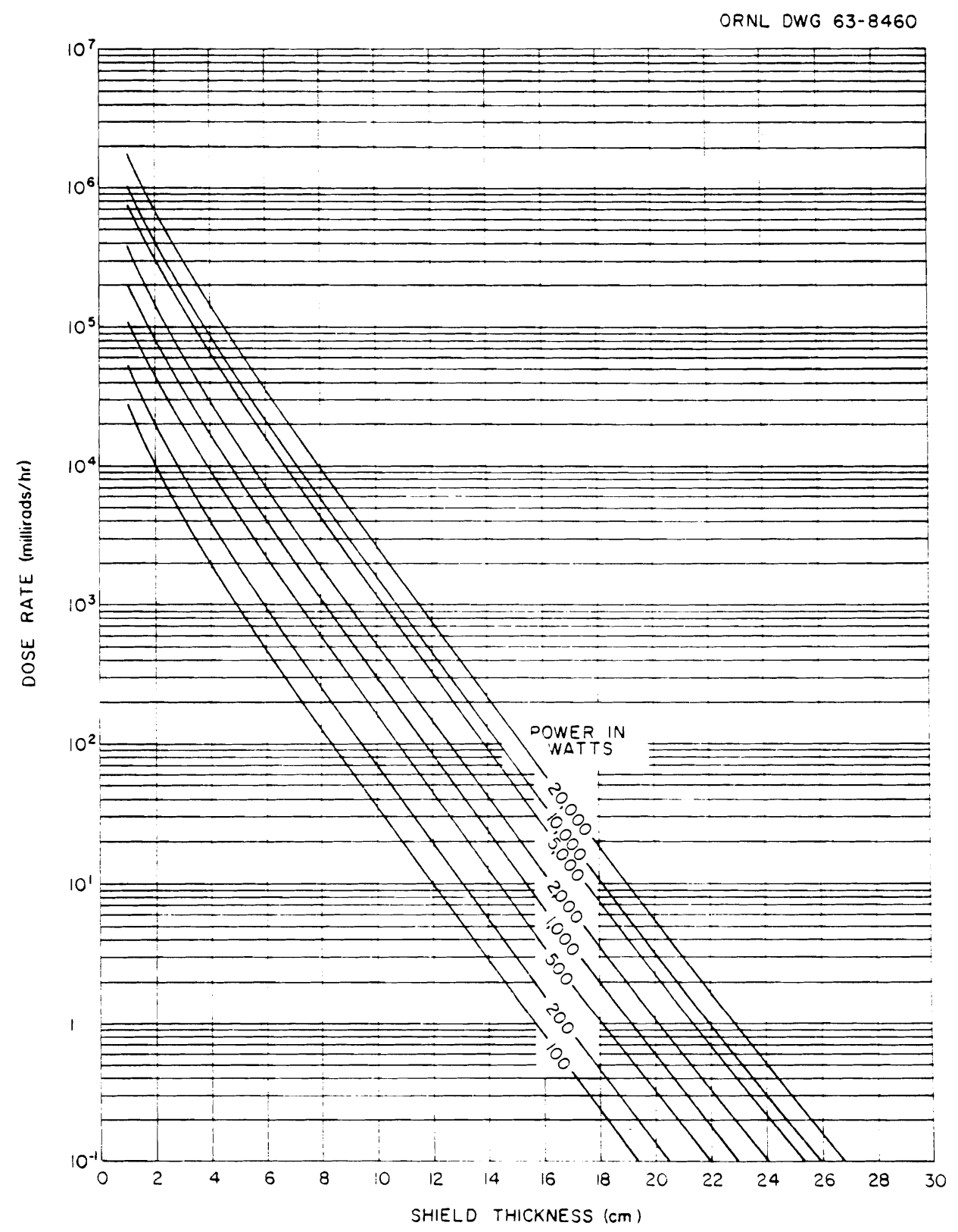

Figure 6. Bremsstrahlung Dose Rates from Lead-Shielded Isotopic Power Sources of Strontium-90 (Strontium Oxjde). Center of source to dose point separation distance $=100 \mathrm{~cm}$. $(12)$ 


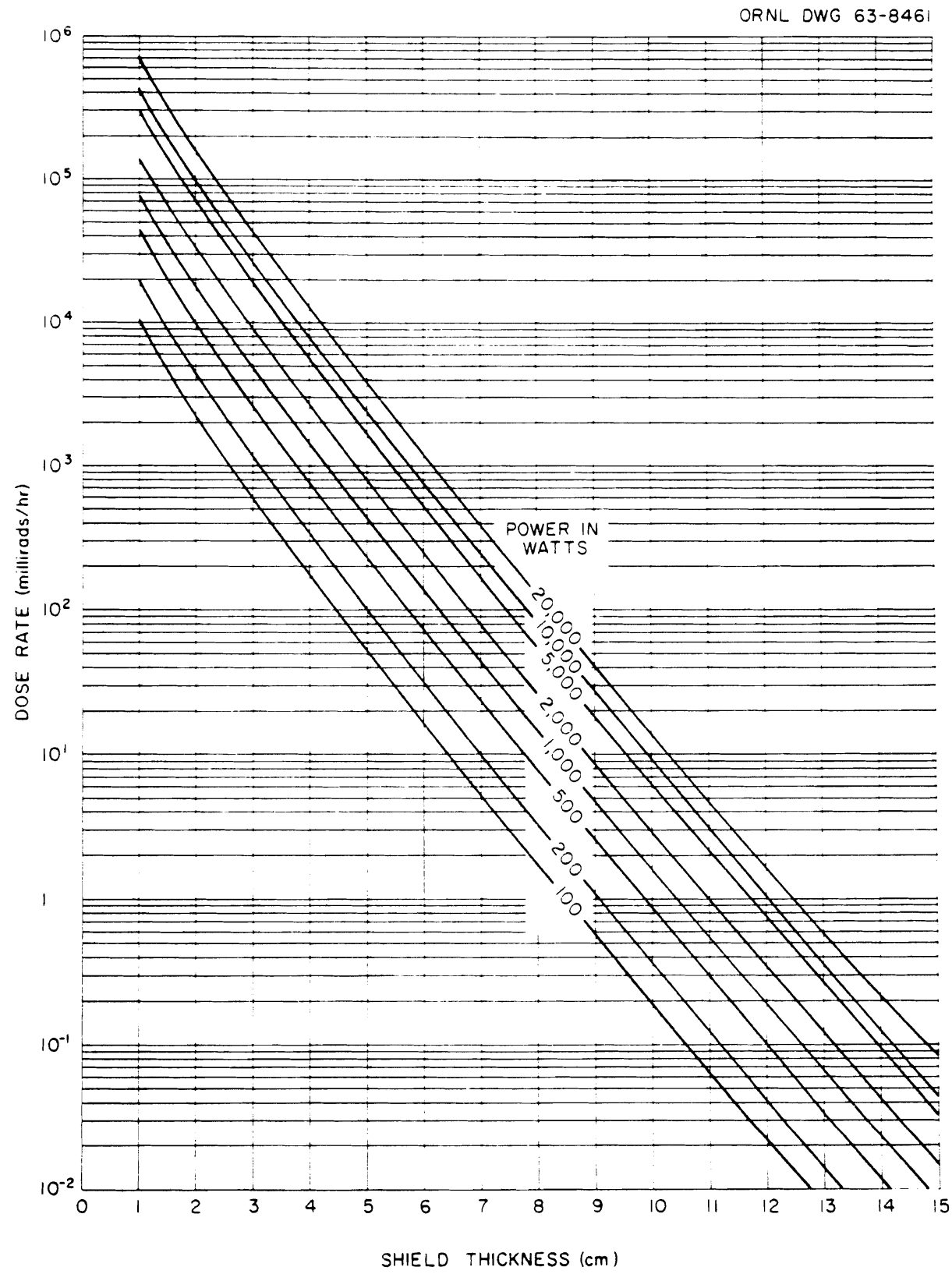

Figure 7. Bremsstrahlung Dose Rates from Uranium-Shielded Isotopic Power Source of Strontium-90 (Strontium Oxide). Center of source to dose point separation distance $=100 \mathrm{~cm}$. (12) 


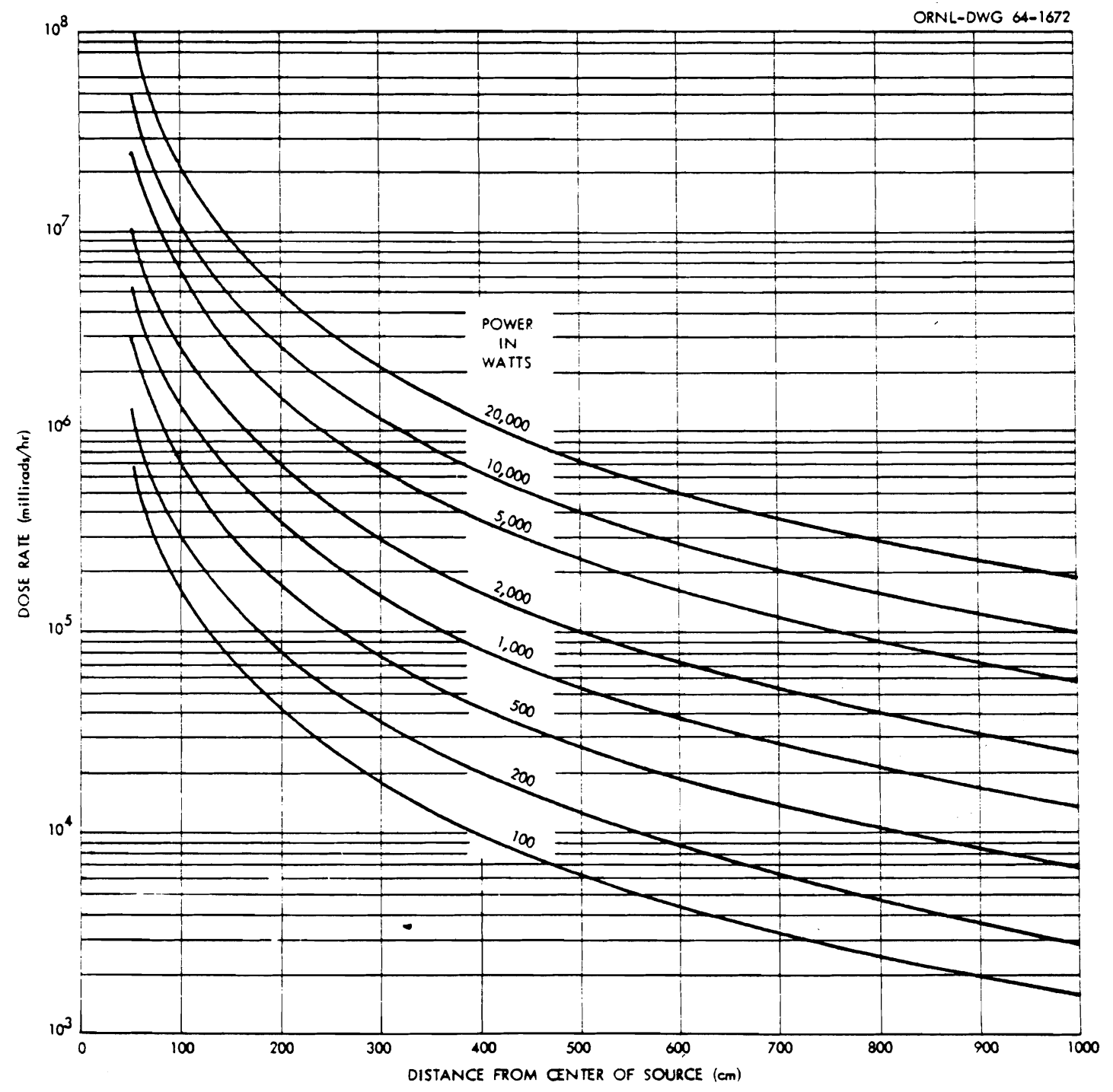

Figure 8. Bremsstrahlung Dose Rates from Unshielded Isotopic Power Sources of Strontium-90 (Strontium Titanate) as a Function of Distance from Center of Source. (12) 


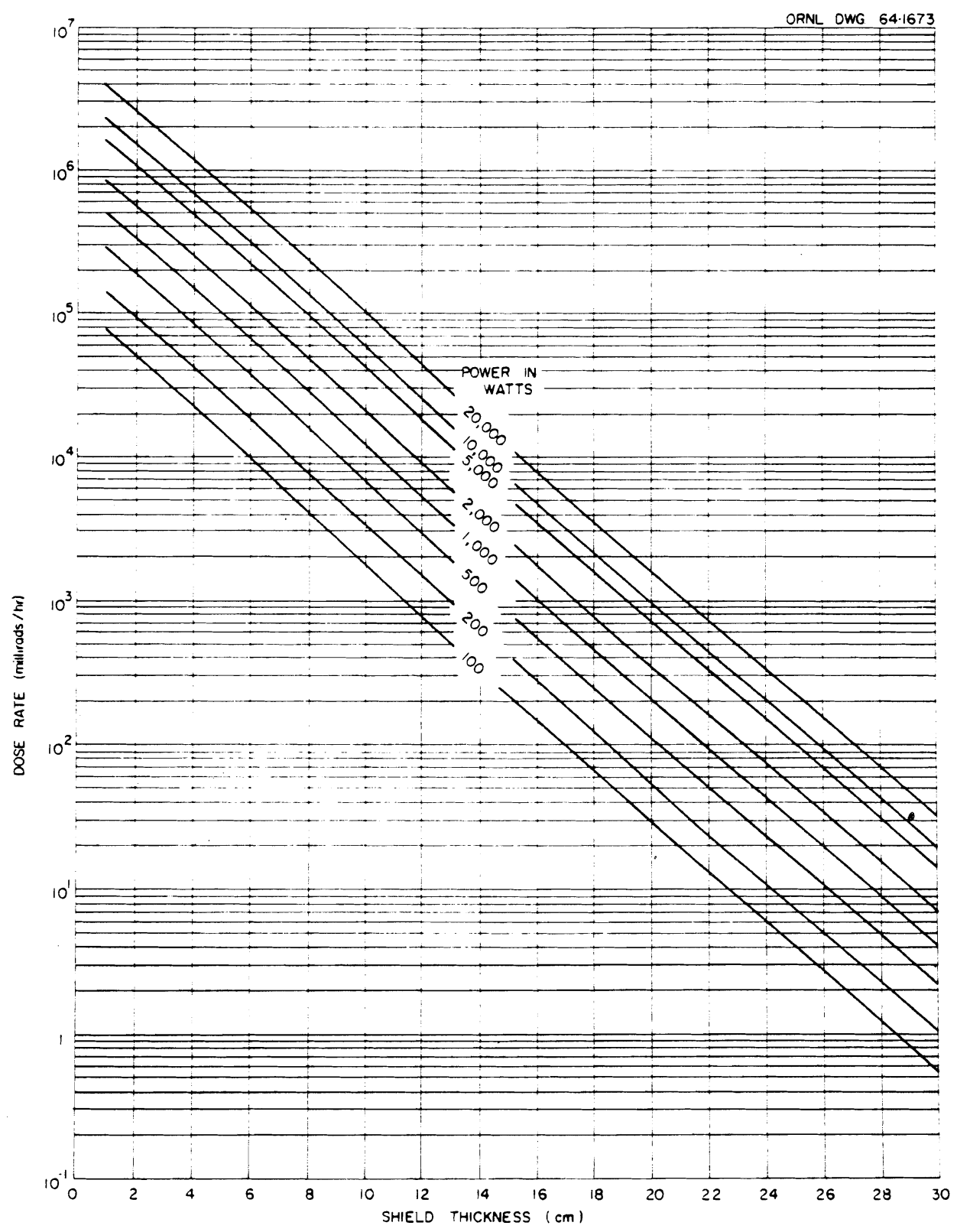

Figure 9. Bremsstrahlung Dose Rates from Iron-Shielded Isotopic Power Sources of Strontium-90 (Strontium Titanate) Center of source to dose point separation distance $=100 \mathrm{~cm} .(12)$ 


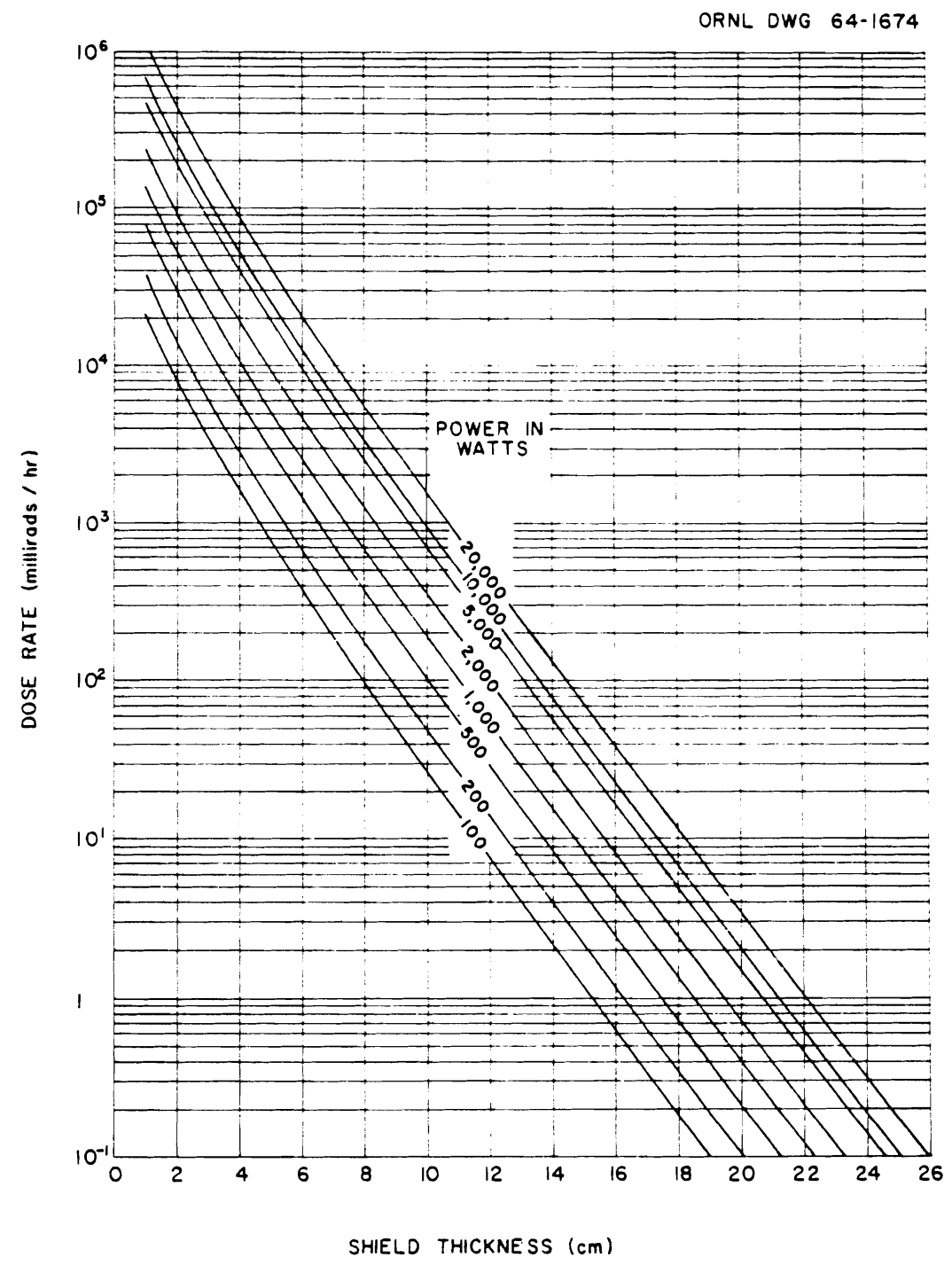

Figure 10. Bremsstrahlung Dose Rates from Lead-Shielded Isotopic Power Sources of Strontium-90 (Strontium Titanate). Center of source to dose point separation distance $=100 \mathrm{~cm}$. 


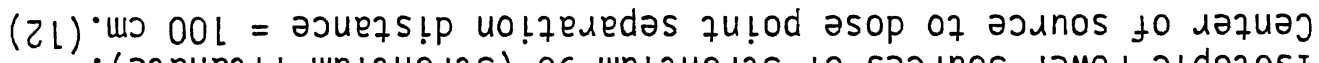

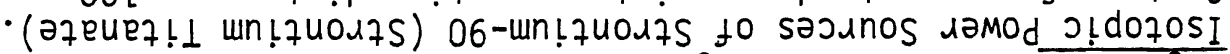

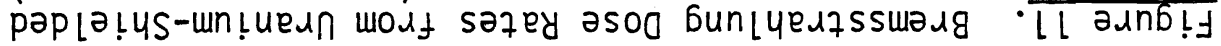

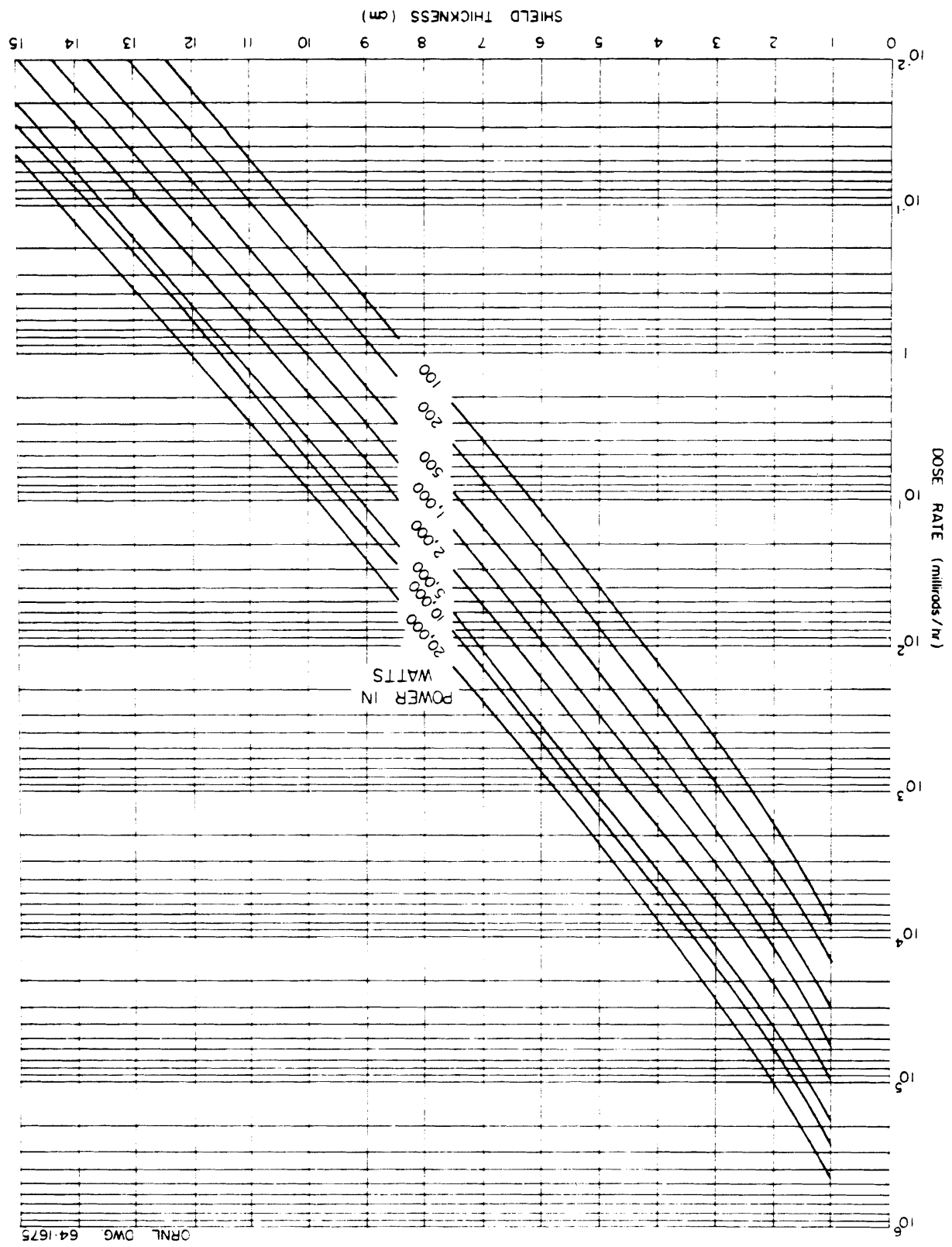




\subsection{BIOLOGICAL TOLERANCES $(14,15,16,17)$}

Nonradioactive strontium fluoride should be handled as a toxic chemical. The biological hazard of the inert strontium fluoride is greatly outweighed by the biological hazard of the strontium-90 fluoride. The strontium-89 and strontium-90 tolerances recommended by the International Commission on Radiological Protection are presented below.

Maximum Permissible Body-Burdens and Maximum Permissible Concentrations of Radionuclides in Air and Water for Occupational Exposure $(14,15)$

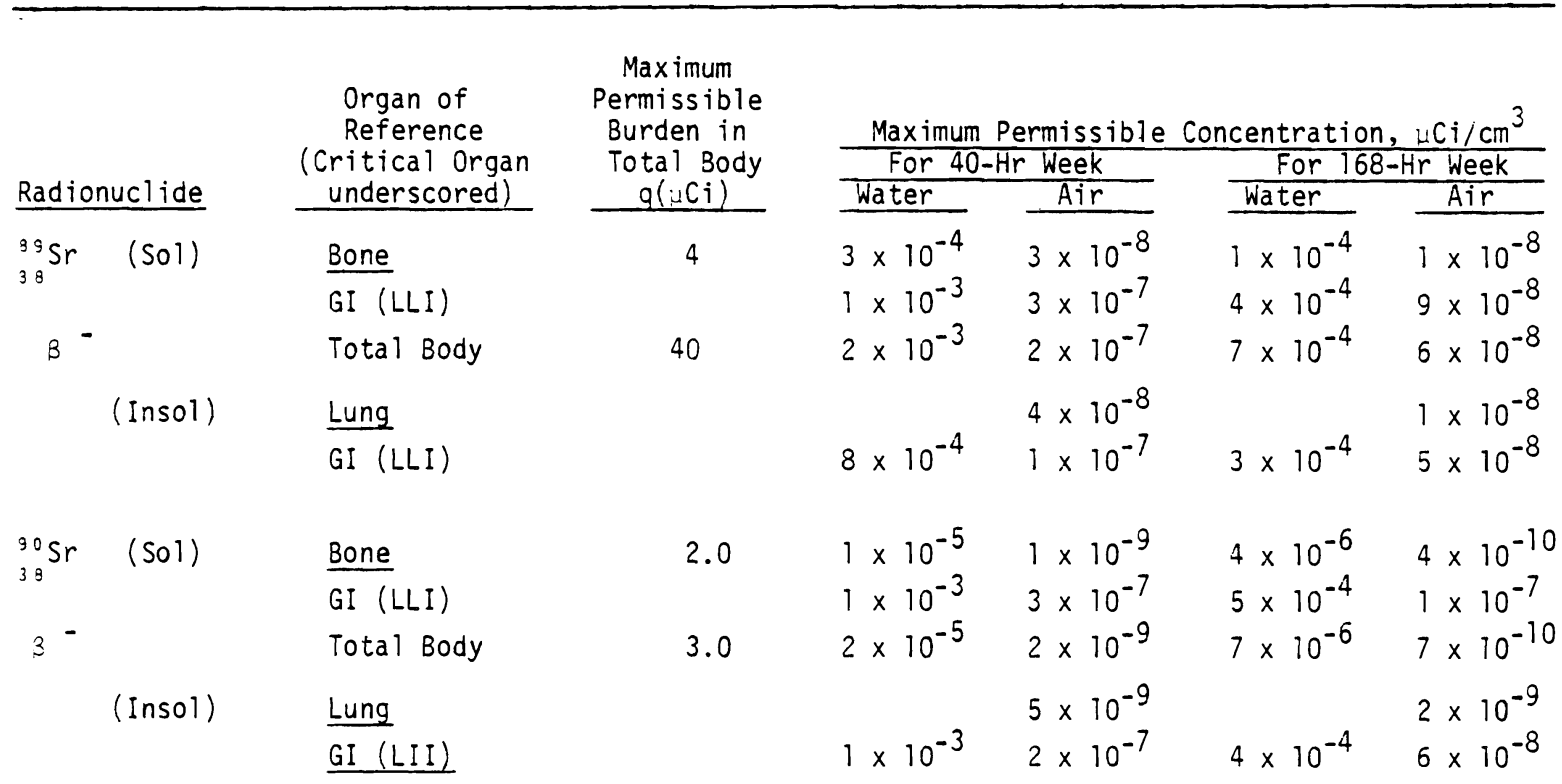

The abbreviations GI and LII refer to the gastrointestinal tract and lower large intestine respectively.

The maximum permissible concentrations for ${ }^{89} \mathrm{Sr}$ and ${ }^{9}{ }^{\circ} \mathrm{Sr}$ in restricted and unrestricted areas as defined in 1OCFR Part 20(16) and ERDA-MC-0524(17) are presented below: 
Maximum Permissible Concentrations for ${ }^{89} \mathrm{Sr}$ and ${ }^{90} \mathrm{Sr}$ in Air and Water Above Natural Background(16)

\begin{tabular}{|c|c|c|c|c|}
\hline \multirow[b]{3}{*}{ Radionuclide } & \multicolumn{4}{|c|}{ Maximum Permissible Concentration, $\mu \mathrm{Ci} / \mathrm{ml}$} \\
\hline & \multicolumn{2}{|c|}{ Restricted Area $(a)$} & \multicolumn{2}{|c|}{ Unrestricted Area } \\
\hline & Water & Air & Water & Air \\
\hline${ }_{38}^{89} \mathrm{Sr} \quad$ (SOI) & $3 \times 10^{-4}$ & $3 \times 10^{-8}$ & $3 \times 10^{-6}$ & $3 \times 10^{-10}$ \\
\hline (Insol) & $8 \times 10^{-4}$ & $4 \times 10^{-8}$ & $3 \times 10^{-5}$ & $1 \times 10^{-9}$ \\
\hline${ }_{38}^{\circ} \mathrm{Sr} \quad$ (Sol) & $1 \times 10^{-5}$ & $1 \times 10^{-9}$ & $3 \times 10^{-7}$ & $3 \times 10^{-11}$ \\
\hline (Insol) & $1 \times 10^{-3}$ & $5 \times 10^{-9}$ & $4 \times 10^{-5}$ & $2 \times 10^{-10}$ \\
\hline
\end{tabular}

(a) Based on a $40-h r$ exposure in any period of seven consecutive days.

The radiation exposure limits for individuals in restricted and unrestricted areas as defined in ERDA-MC-0524(17) are presented below:

Exposure Limits for Individuals in Unrestricted Areas (17)

\begin{tabular}{|c|c|c|}
\hline Type of Exposure & $\begin{array}{l}\text { Annual Dose } \\
\text { or Dose Commi } \\
\text { Based on dose } \\
\text { to ind ividuals at } \\
\text { points of maximum } \\
\text { probable exposure }\end{array}$ & $\begin{array}{l}\text { Equivalent } \\
\text { ment (rem) } \\
\text { Based on an average } \\
\text { dose to a suitable } \\
\text { sample of the } \\
\text { exposed population }\end{array}$ \\
\hline $\begin{array}{l}\text { Whole body, gonads, } \\
\text { or bone marrow }\end{array}$ & 0.5 & 0.17 \\
\hline Other organs & 1.5 & 0.5 \\
\hline $\begin{array}{l}\text { (a) In keeping with } \\
\text { as expressed in } 0 \\
\text { shall be limited } \\
\text { annual dose limit }\end{array}$ & $\begin{array}{l}\text { A policy on lowes } \\
\text { pter } 0524-012 \text {, ex } \\
\text { as small a fract } \\
\text { as is practicable }\end{array}$ & $\begin{array}{l}\text { practicable exposure } \\
\text { sures to the public } \\
\text { in of the respective }\end{array}$ \\
\hline
\end{tabular}


Exposure Limits for Individuals in Restricted Areas

(17)

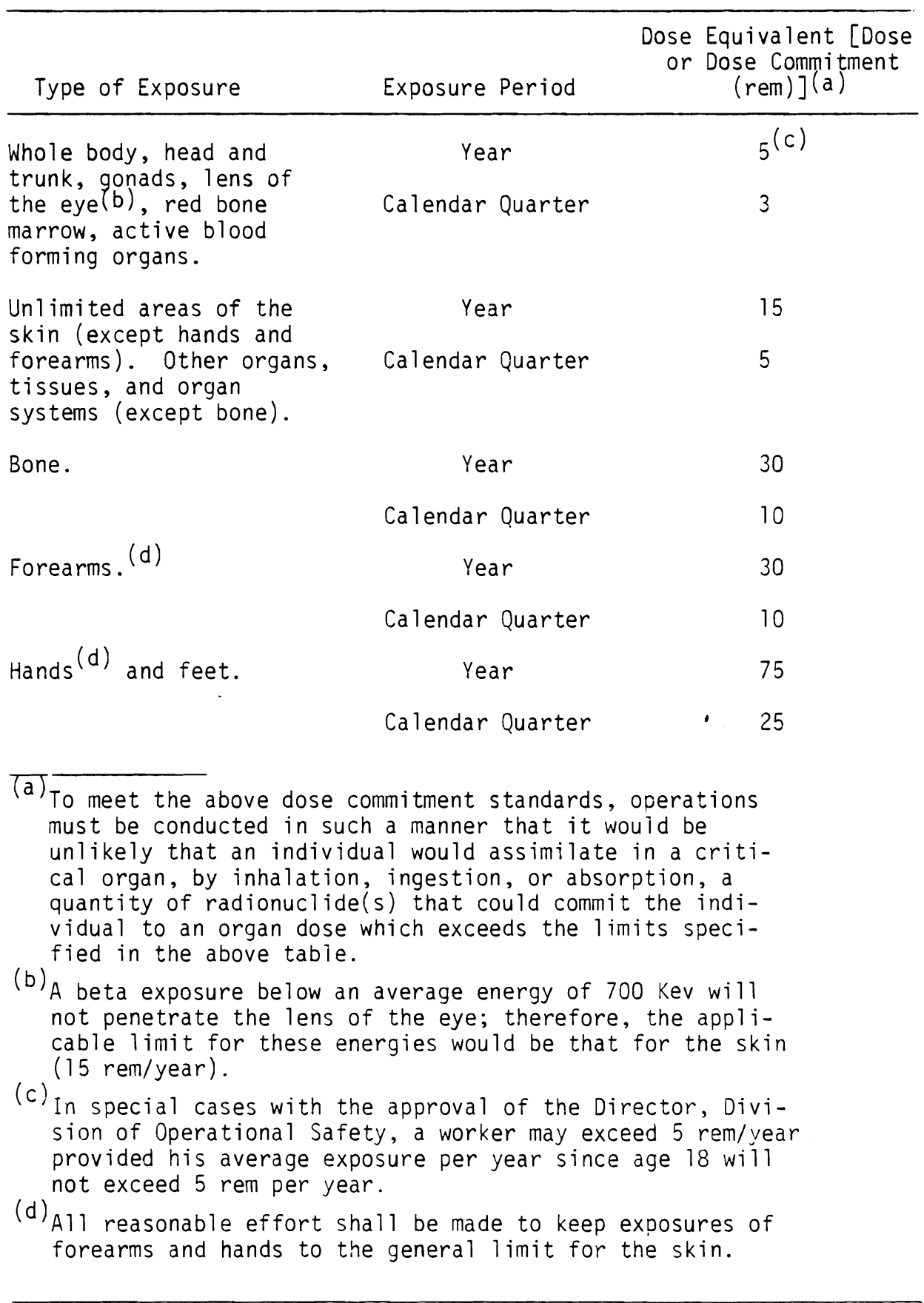




\subsection{CRITICAL MASS}

The radioactive strontium isotopes and ${ }^{90} \mathrm{Y}$ are not fissionable.

\subsection{THERMOPHYSICAL PROPERTIES}

\subsubsection{Density}

Pure $\mathrm{SrF}_{2}$

$$
\rho=4.29 \mathrm{~g} / \mathrm{cm}^{3}\left(\text { at } 25^{\circ} \mathrm{C}\right)
$$

WESF-Grade $\mathrm{SrF}_{2}$ (calculated value)

$$
\rho=4.24 \mathrm{~g} / \mathrm{cm}^{3}\left(\text { at } 25^{\circ} \mathrm{C}\right)
$$

Pure $\mathrm{SrF}_{2}$ - Molten (19)

$$
\begin{aligned}
& \rho= 4.784-7.51 \times 10^{-4} \mathrm{~T} \mathrm{~g} / \mathrm{cm}^{3}\left(1750^{\circ}-2200^{\circ} \mathrm{C}\right) \\
& \text { Where } T \text { is the temperature in }{ }^{\circ} \mathrm{K}
\end{aligned}
$$

Figure 12 shows the density of strontium fluoride from $298^{\circ} \mathrm{K}$ to $2200^{\circ} \mathrm{K}$. The density of solid strontium fluoride from $298^{\circ} \mathrm{K}$ to $973^{\circ} \mathrm{K}$ was calculated using the thermal expansion coefficient given in Section 3.9.2. Figure 13 shows the experimental values from which the density equation for molten strontium fluoride was determined.

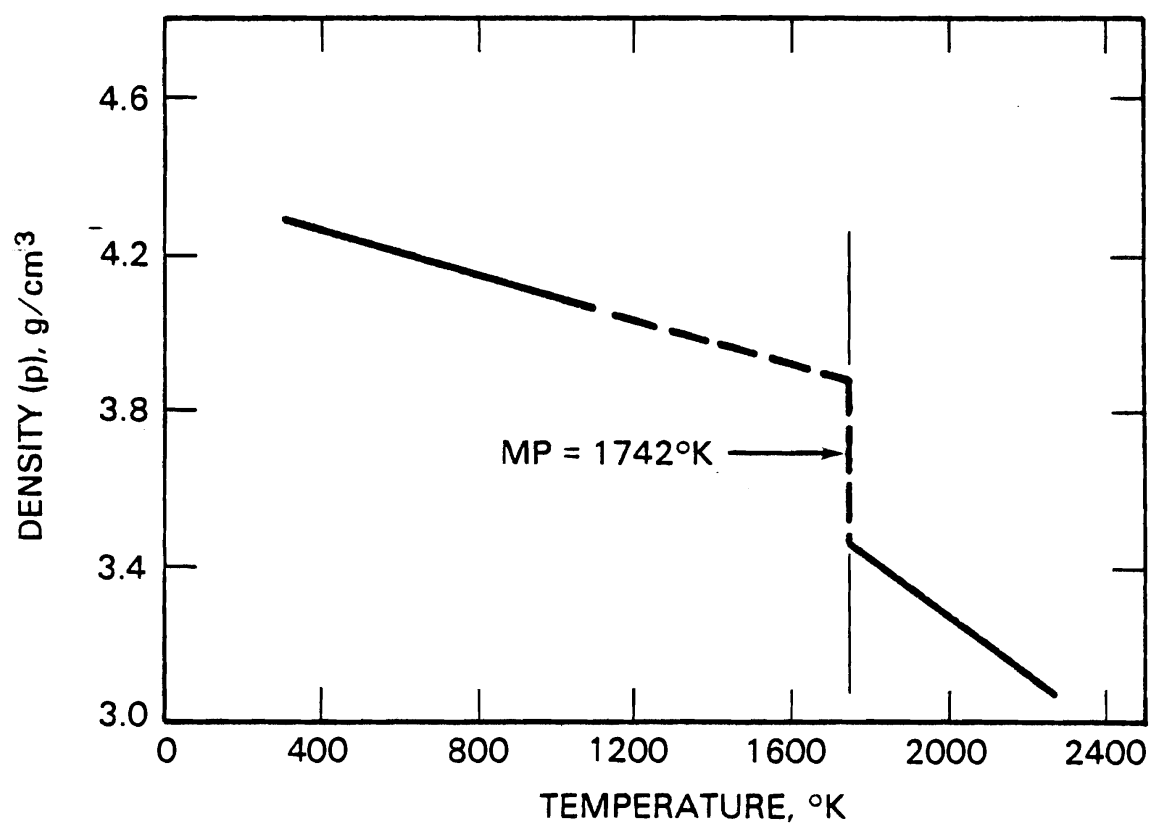

FIGURE 12. Density of Strontium Fluoride. 


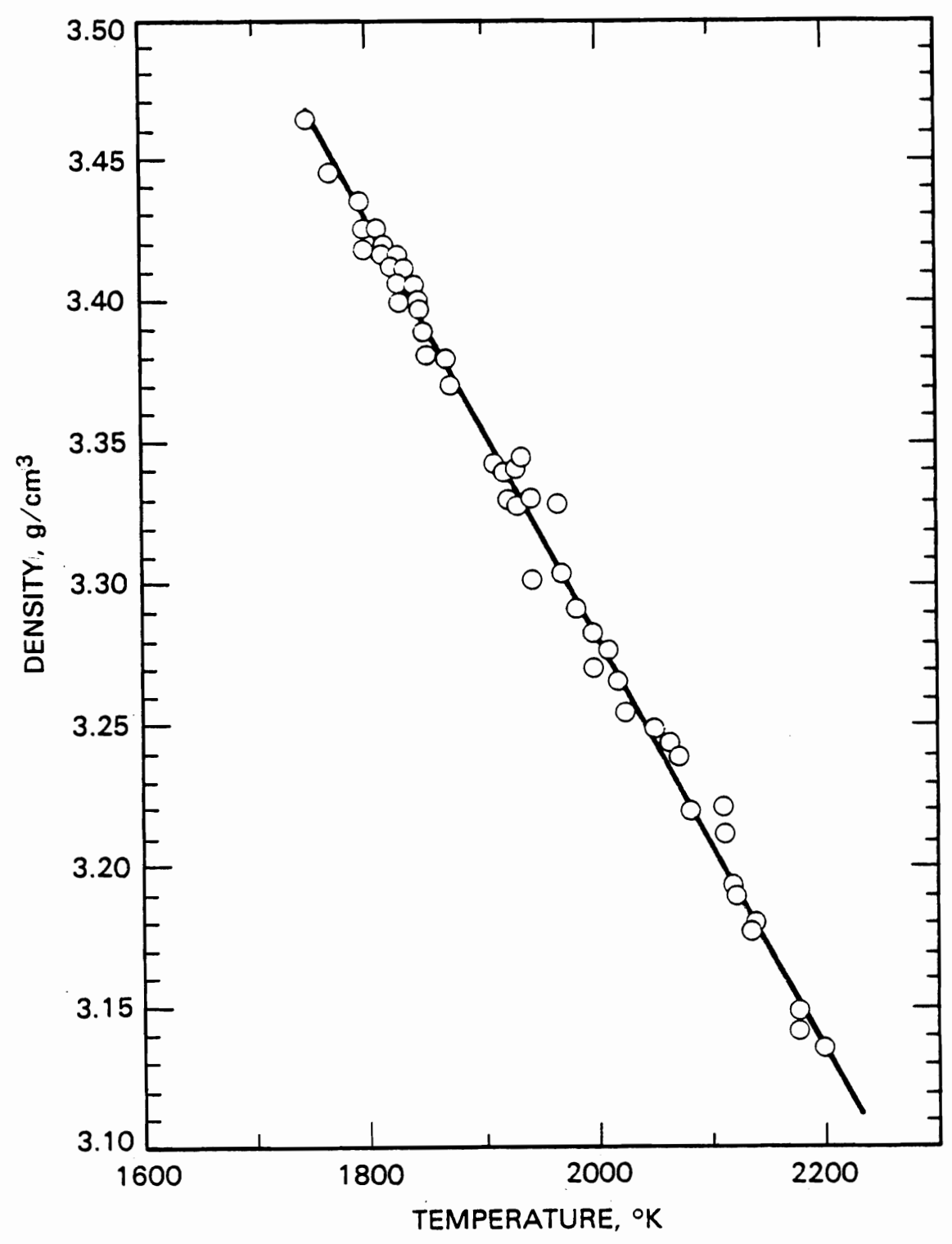

FIGURE 13. Density of Molten Strontium Fluoride (19). 
3.9.2 Thermal Expansion (20)

The thermal expansion of pure strontium fluoride and WESF-grade strontium fluoride was measured over a temperature range of $50^{\circ}-700^{\circ} \mathrm{C}$. The thermal expansion coefficient a was essentially constant over the temperature range studied.

\begin{tabular}{|c|c|c|}
\hline Grade of $\mathrm{SrF}_{2}$ & $\begin{array}{l}\text { Temperature } \\
{ }^{\circ} \mathrm{C}\end{array}$ & $\begin{array}{l}\text { Linear Thermal Expansion } \\
\text { Coefficient }(\alpha), \mathrm{cm} / \mathrm{cm}-{ }^{\circ} \mathrm{C}\end{array}$ \\
\hline Pure $\mathrm{SrF}_{2}$ & $50-700$ & $2.13 \times 10^{-5}$ \\
\hline $\begin{array}{l}\text { WESF-Grade } \\
\text { SrF } 2\end{array}$ & $50-700$ & $2.19 \times 10^{-5}$ \\
\hline
\end{tabular}

\subsubsection{Specific Heat $(20,21,22,23)$}

The specific heat of pure strontium fluoride has been determined experimentally by various investigators over a temperature range of $100^{\circ}$ to $2126^{\circ} \mathrm{K}$. The specific heat of WESF-grade strontium fluoride was measured over a temperature range of $320^{\circ}$ to $710^{\circ} \mathrm{K}$. The experimentally measured values for the two grades of strontium fluoride are presented in the following table. 
The Specific Heat of Pure $\mathrm{SrF}_{2}$ and WESF-Grade $\mathrm{SrF}_{2}$

\begin{tabular}{|c|c|c|c|c|}
\hline \multirow[b]{2}{*}{$\underset{{ }^{\circ} \mathrm{K}}{\text { Temperature }}$} & \multicolumn{4}{|c|}{ Specific Heat $\left(C_{p}\right)$, cal $/ g-{ }^{\circ} C$} \\
\hline & $\begin{array}{c}\text { Pure } \mathrm{SrF}_{2} \\
(22)\end{array}$ & $\begin{array}{c}\text { Pure SrF } 2 \\
(21,23)^{2}\end{array}$ & $\begin{array}{l}\text { Pure } \mathrm{SrF}_{2} \\
\text { (20) }\end{array}$ & $\begin{array}{l}\text { WESF-Grade } \\
\mathrm{SrF}_{2}(20)\end{array}$ \\
\hline 0 & 0 & & & \\
\hline 100 & 0.0698 & & & \\
\hline 200 & 0.1173 & & & \\
\hline 298 & 0.1332 & & {$[0.1307]$} & {$[0.1361]$} \\
\hline 320 & & & 0.1340 & 0.1395 \\
\hline 360 & & & 0.1384 & 0.1442 \\
\hline 400 & & & 0.1417 & 0.1477 \\
\hline 440 & & & 0.1443 & 0.1504 \\
\hline 480 & & & 0.1468 & 0.1526 \\
\hline 520 & & & 0.1491 & 0.1537 \\
\hline 560 & & & 0.1510 & 0.1537 \\
\hline 600 & & & 0.1510 & 0.1537 \\
\hline 700 & & & 0.1510 & 0.1537 \\
\hline 800 & & 0.1540 & & \\
\hline 900 & & 0.1567 & & \\
\hline 1000 & & 0.1632 & & \\
\hline 1100 & & 0.1716 & & \\
\hline 1200 & & 0.1847 & & \\
\hline 1300 & & 0.2070 & & \\
\hline 1400 & & 0.2738 & & \\
\hline 1500 & & 0.2945 & & \\
\hline 1600 & & 0.2396 & & \\
\hline 1700 & & 0.2165 & & \\
\hline 1800 & & 0.2042 & & \\
\hline 1900 & & 0.1954 & & \\
\hline 2000 & & 0.1895 & & \\
\hline 2100 & & 0.1859 & & \\
\hline
\end{tabular}

[ ] Extrapolated values 
3.9.4 Debye Temperature ${ }^{(24)}$

$$
\theta_{D}=420^{\circ} \mathrm{K} \text { (estimated value) }
$$

\subsubsection{Enthalpy}

The enthalpies, or heat contents, of pure $\mathrm{SrF}_{2}$ and WESF-Grade $\mathrm{SrF}_{2}$ were calculated from the specific heat data given in Section 3.9.3 using the equation

$$
H_{t}^{\circ}-H_{298}^{\circ}=\int_{298}^{t} C_{p} d t
$$

The average molecular weight of the WESF-Grade $\mathrm{SrF}_{2}$ was assumed

\begin{tabular}{|c|c|c|}
\hline \multirow{2}{*}{$\begin{array}{c}\text { Temperature } \\
{ }^{\circ} \mathrm{K}\end{array}$} & \multicolumn{2}{|c|}{$H_{t}^{\circ}-H_{298}^{\circ}, \mathrm{cal} / \mathrm{g}$ mole } \\
\hline & Pure $\mathrm{SrF}_{2}$ & WESF-Grade $\mathrm{SrF}_{2}$ \\
\hline 298 & 0 & 0 \\
\hline 400 & 1,750 & 1,810 \\
\hline 500 & 3,570 & 3,690 \\
\hline 600 & 5,460 & 5,600 \\
\hline 700 & 7,360 & 7,520 \\
\hline 800 & 9,260 & 9,400 \\
\hline 900 & 11,220 & \\
\hline 1,000 & 13,230 & \\
\hline 1,100 & 15,320 & \\
\hline 1,200 & 17,560 & \\
\hline 1,300 & 19,990 & \\
\hline 1,400 & 22,920 & \\
\hline 1,500 & 26,800 & \\
\hline 1,600 & 30,080 & \\
\hline 1,700 & 32,930 & \\
\hline 1,800 & 35,560 & \\
\hline 1,900 & 39,060 & \\
\hline 2,000 & 40,480 & \\
\hline 2,100 & 42,830 & \\
\hline
\end{tabular}
to be 124.5 . 
Enthalpy of Pure Strontium Fluoride (21)

\begin{tabular}{|c|c|c|}
\hline \multirow[b]{2}{*}{ Temperature, ${ }^{\circ} \mathrm{K}$} & \multicolumn{2}{|c|}{$H_{t}^{\circ}-H_{298}^{\circ}, \mathrm{kcal} / \mathrm{mol}$} \\
\hline & Liquid & Ideal Gas \\
\hline 0 & & -3.19 \\
\hline 100 & & -2.29 \\
\hline 200 & & -1.20 \\
\hline 298 & 0 & 0 \\
\hline 400 & 1.77 & 1.32 \\
\hline 500 & 3.59 & 2.65 \\
\hline 600 & 5.45 & 4.00 \\
\hline 700 & 7.35 & 5.36 \\
\hline 800 & 9.27 & 6.72 \\
\hline 900 & 11.22 & 8.10 \\
\hline 1000 & 13.23 & 9.47 \\
\hline 1100 & 15.32 & 10.85 \\
\hline 1200 & 17.56 & 12.23 \\
\hline 1300 & 19.92 & 13.61 \\
\hline 1400 & 22.29 & 15.00 \\
\hline 1500 & 24.66 & 16.38 \\
\hline 1600 & 27.03 & 17.77 \\
\hline 1700 & 29.40 & 19.15 \\
\hline 1800 & 31.76 & 20.54 \\
\hline 1900 & 34.13 & 21.93 \\
\hline 2000 & 36.50 & 23.32 \\
\hline 2200 & 41.23 & 26.09 \\
\hline 2400 & 45.57 & 28.87 \\
\hline 2600 & 55.43 & 31.64 \\
\hline 2800 & 55.43 & 34.42 \\
\hline 3000 & 60.16 & 37.20 \\
\hline 3200 & 64.90 & 39.98 \\
\hline 3400 & 69.64 & 42.76 \\
\hline
\end{tabular}




\subsubsection{Phase Transitions}

a. Solid Phase Transitions $(21,23,24)$

$\mathrm{SrF}_{2}$ is reported to undergo a phase transition between $1421^{\circ}$ and $1484^{\circ} \mathrm{K}$. The phase transition has not been verified by $X$-ray diffraction measurements; however, the transition appears to be a second order transition similar to that observed with other alkaline earth halides having the calcium fluoride structure. (24)

b. Melting Point

A range of values has been reported in the literature for the melting point of $\mathrm{SrF}_{2}$. Recently reported values for pure $\mathrm{SrF}_{2}$ are given below:

\begin{tabular}{|c|c|}
\hline Source & $\begin{array}{c}\text { Melting Point } \\
{ }^{\circ} \mathrm{K} \\
\end{array}$ \\
\hline Efremova and Matizen (23) & $1750 \pm 2$ \\
\hline Kojima, et a1. (25) & $1746 \pm 1$ \\
\hline Petit and Delbove (26) & 1735 \\
\hline \multirow[t]{2}{*}{ Porter and Brown (27) } & $1736 \pm 5$ \\
\hline & $1742^{\circ} \mathrm{K}$ \\
\hline
\end{tabular}

Additions of impurities to the strontium fluoride will reduce the melting point. The $\mathrm{SrF}_{2}-\mathrm{NaF}$ system, for example, exhibits a mirimum melting point of $856^{\circ} \mathrm{C}(28)$. Figure 14 shows the phase diagram for the system. Differential thermal analysis of WESF-grade strontium fluoride shows the existence of a low melting phase having a melting point of $851^{\circ} \pm 5^{\circ} \mathrm{C}$ (29). At temperatures up to $1100^{\circ} \mathrm{C}$ the amount of 1 iquid phase present appears to be very smal1 $(<7 \%)$.

c. Boiling Point (21)

$T_{b}=2759^{\circ} \mathrm{K}$ 


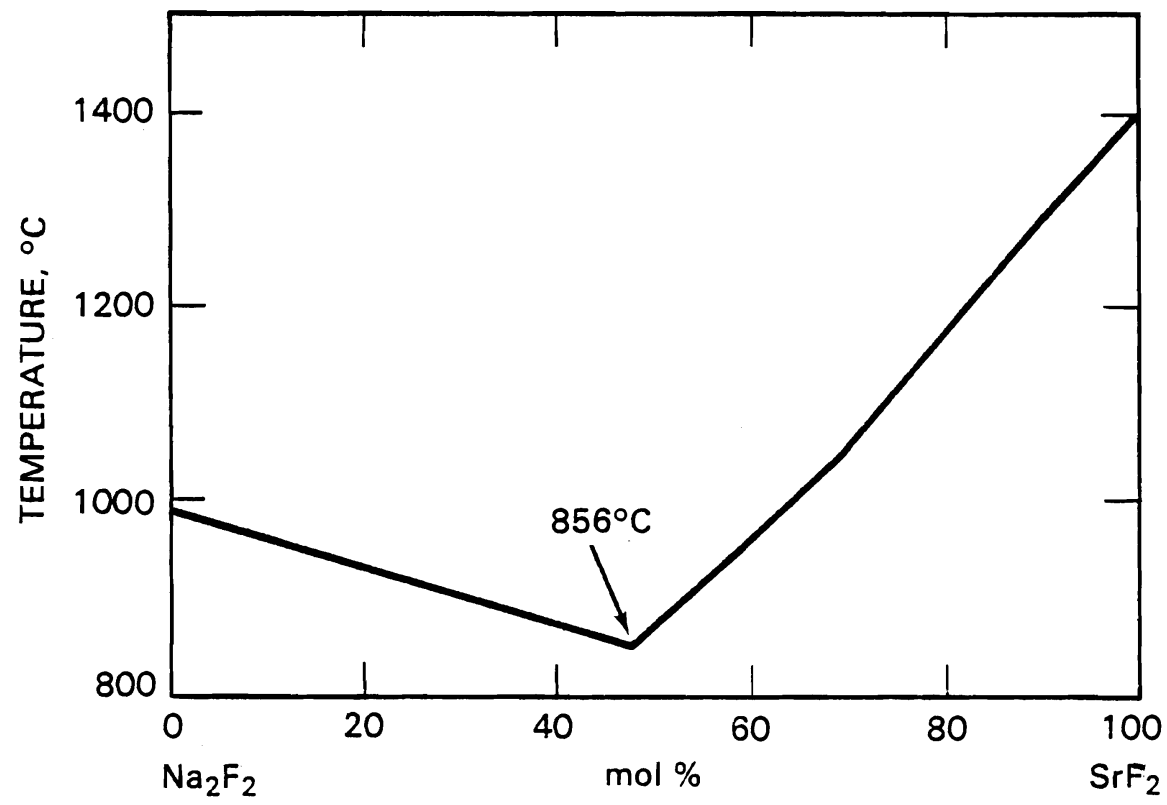

Figure 14. Phase Diagram for the System $\mathrm{SrF}_{2}-\mathrm{Na}_{2} \mathrm{~F}_{2}$ (28).

\subsubsection{Latent Heats of Phase Transitions}

a. Solid Phase Transition (21)

$\Delta H_{t} \simeq 0$

b. Melting (21)

$\Delta H_{m}=7.09 \pm 0.15 \mathrm{kcal} / \mathrm{mol}$

c. Boiling (21)

$\Delta H_{V}=76.4 \mathrm{kcal} / \mathrm{mol}$ 
3.9.8 Vapor Pressure ${ }^{(30)}$

\begin{tabular}{cc}
\hline $\begin{array}{c}\text { Temperature } \\
{ }^{\circ} \mathrm{C}\end{array}$ & $\begin{array}{c}\text { Vapor Pressure } \\
\text { Torr }\end{array}$ \\
1822 & 8.5 \\
1880 & 15.0 \\
1927 & 25.7 \\
1959 & 31.0 \\
\hline
\end{tabular}

3.9.9 Thermal Diffusivity (20)

The thermal diffusivities of pure $\mathrm{SrF}_{2}$ and WESF-Grade $\mathrm{SrF}_{2}$ were measured over a temperature range of $50^{\circ}-850^{\circ} \mathrm{C}$ using a pulsed laser technique.

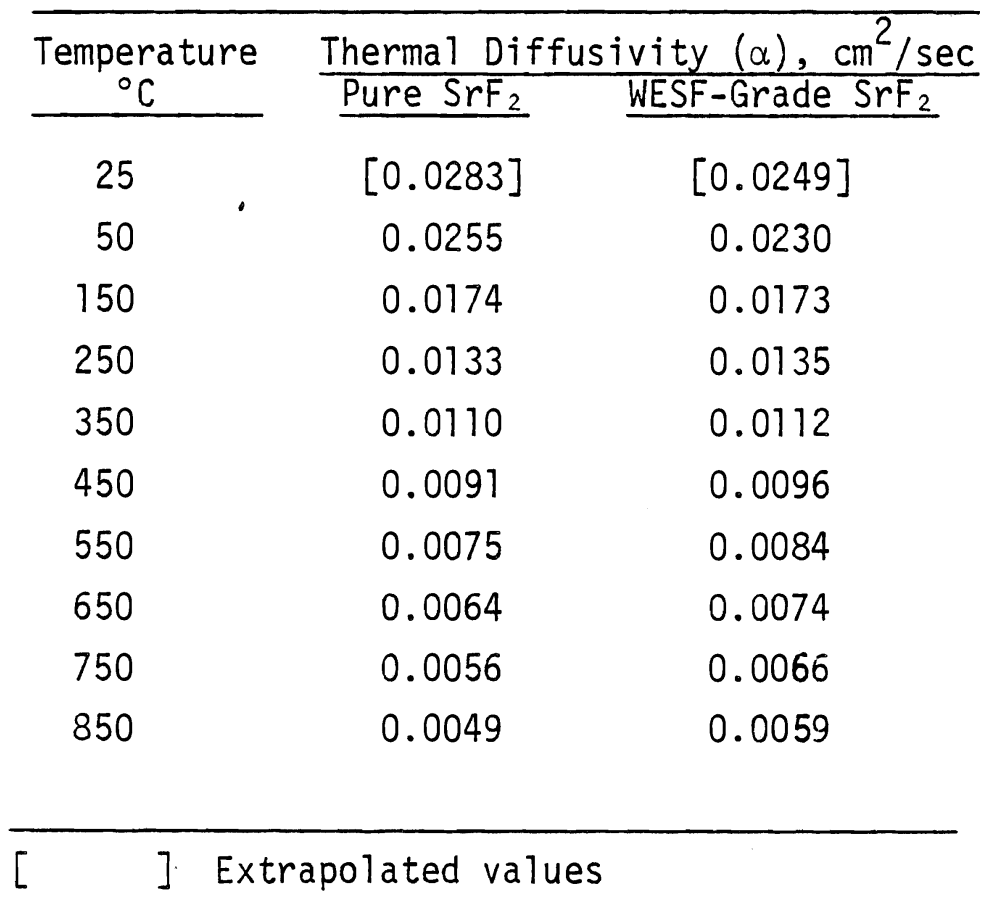




\subsubsection{Thermal Conductivity}

The thermal conductivities of pure $\mathrm{SrF}_{2}$ and WESF-Grade $\mathrm{SrF}_{2}$ were calculated from the thermal diffusivity values given in Section 3.9.9 using the equation

$$
\lambda=\alpha \cdot C_{p} \cdot \rho
$$

where $\lambda$ is the thermal conductivity in $\mathrm{cal} / \mathrm{cm}-\mathrm{sec}-{ }^{\circ} \mathrm{C}$

$\alpha$ is the thermal diffusivity in $\mathrm{cm}^{2} / \mathrm{sec}$

$C_{p}$ is the specific heat in $\mathrm{cal} / \mathrm{g}-{ }^{\circ} \mathrm{C}$

$\rho$ is the density in $\mathrm{g} / \mathrm{cm}^{3}$.

The values of $C p$ used in calculating $\lambda$ are given in Section 3.9.3. The density values were calculated using the thermal expansion coefficients given in Section 3.9.2.

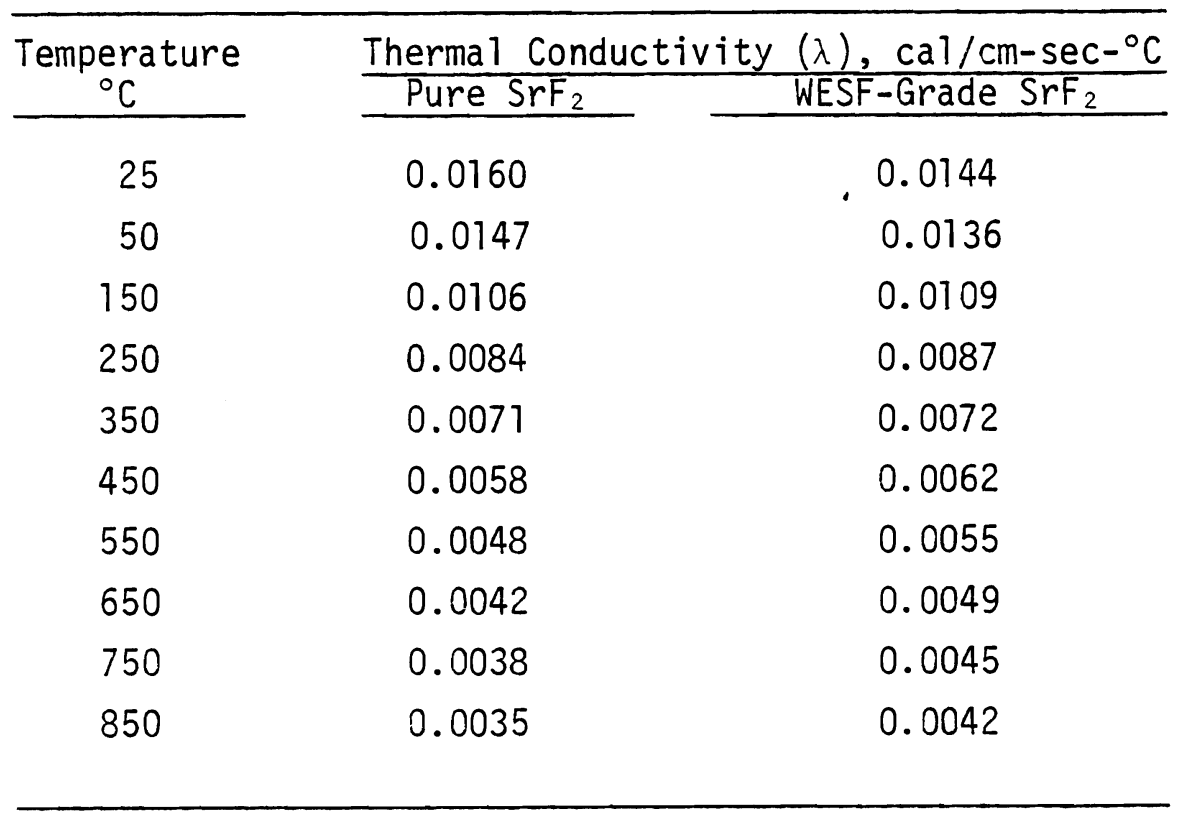


3.9.11 Viscosity (3)

$$
\begin{aligned}
& \log \mu=0.781+436 / T \\
& \text { where } \mu \text { is the fluid viscosity in centipoises } \\
& T \text { is the temperature in }{ }^{\circ} \mathrm{K} .
\end{aligned}
$$

\subsubsection{Surface Tension (31)}

$$
670-0.3 T \mathrm{erg} / \mathrm{cm}^{2}
$$

where $T$ is the temperature in ${ }^{\circ} \mathrm{C}$.

\subsubsection{Total Hemispherical Emittance}

No value reported

3.9.14 Spectral Emissivity (3)

A value of 0.9 is recommended.

\subsubsection{Crystal lography (32)}

Face centered cubic - $\mathrm{Cl}\left(\mathrm{CaF}_{2}\right)$ type

$$
\begin{aligned}
& a^{\circ}=5.7996 \AA \\
& A-B \text { distance } 2.51 \AA
\end{aligned}
$$

\begin{tabular}{|c|c|c|}
\hline $\begin{array}{c}\text { Temperature } \\
{ }_{\circ}^{\circ} \mathrm{C} \\
\end{array}$ & $\begin{array}{c}\text { Solubility } \\
\mathrm{g} / \mathrm{l}\end{array}$ & $\begin{array}{c}\text { Solubility Product } \\
\text { Ksp }\end{array}$ \\
\hline 4 & 0.133 & $4.7 \times 10^{-9}$ \\
\hline 23 & 0.135 & $5.1 \times 10^{-9}$ \\
\hline 35 & 0.143 & $5.8 \times 10^{-9}$ \\
\hline 50 & 0.157 & $7.7 \times 10^{-9}$ \\
\hline
\end{tabular}

\subsubsection{Solubility in Aqueous Media (33)}

a. Pure $\mathrm{SrF}_{2}$

Solubility of Pure $\mathrm{SrF}_{2}$ in Demineralized Water (a)

(a) Demineralized water having a resistivity of 2 megohms 
Solubility of Pure $\mathrm{SrF}_{2}$ in Natural Seawater

\begin{tabular}{cccc}
\hline $\begin{array}{c}\text { Temperature } \\
{ }^{\circ} \mathrm{C}\end{array}$ & $\begin{array}{c}\text { Equilibrium } \\
\text { Sr Conc. } \\
M\end{array}$ & $\begin{array}{c}\text { Equilibrium } \\
\text { F Conc. } \\
M\end{array}$ & $\begin{array}{c}\operatorname{SrF}_{2}(a) \\
\text { Dissolved } \\
\mathrm{g} / 1\end{array}$ \\
\hline 4 & 0.00115 & 0.00227 & 0.114 \\
23 & 0.00119 & 0.00255 & 0.149 \\
35 & 0.00129 & 0.00243 & 0.150 \\
50 & 0.00139 & 0.00268 & 0.163
\end{tabular}

(a) Calculated from the equilibrium $\mathrm{Sr}$ and $\mathrm{F}$ concentrations after correcting for the initial $\mathrm{Sr}$ and $\mathrm{F}$ concentrations of the natural seawater.

b. WESF-Grade $\mathrm{SrF}_{2}$

Experimental studies show that soluble impurities in WESFGrade $\mathrm{SrF}_{2}$ have a signficant effect on the solubility of the $\mathrm{SrF}_{2}$ in demineralized water, both by the common ion effect and by their effect on the ionic strength of the solution. However, the impurities have little effect on the solubility of the $\mathrm{SrF}_{2}$ in seawater.

The Effect of Impurities on the Solubility of Strontium Fluoride in Aqueous Media at $23^{\circ} \mathrm{C}$

\begin{tabular}{lccc}
\hline Grade of $\mathrm{SrF}_{2}(\mathrm{a})$ & \multicolumn{2}{c}{$\mathrm{SrF}_{2}$ DisSolved, $\mathrm{g} / \mathrm{T}$} \\
Pure $\mathrm{SrF}_{2}$ & 0.135 & $\frac{\text { Demineralized Water }}{(b)}$ & Natural Seawater $(\mathrm{c})$ \\
Commercial $\mathrm{SrF}_{2}$ & 0.0749 & 0.149 \\
WESF-Grade $\mathrm{SrF}_{2}$ & 0.0138 & 0.141
\end{tabular}

(a) 15 grams of solid $\mathrm{SrF}_{2}$ added per liter of water

(b) calculated from the equilibrium $\mathrm{Sr}$ concentration

(c) calculated from the equilibrium sr concentration after correcting for the initial Sr concentration of the seawater. 


\subsubsection{Dissolution Behavior}

(33)

The dissolution rate of strontium fluoride in aqueous media is influenced by several factors including:

- the surface area of the $\mathrm{SrF}_{2}$

- the thermal history of the $\mathrm{SrF}_{2}$

- the impurity content of the $\mathrm{SrF}_{2}$

- the composition of the dissolving medium and

- the temperature of the dissolving medium.

The surface area and thermal history of the $\mathrm{SrF}_{2}$ are interrelated, and the relationship is highly dependent on the impurity content of the fluoride.

It was not possible to measure the surface area of the ${ }^{90} \mathrm{SrF}_{2}$, and the dissolution rates for ${ }^{9}{ }^{0} \mathrm{SrF}_{2}$ were reported on the basis of contained strontium ( $\mu \mathrm{gSr}$ dissolved/hr-g Sr initially present). Since the surface area of ${ }^{90} \mathrm{SrF}_{2}$ is not known, direct comparisons of the dissolution rates of ${ }^{90} \mathrm{SrF}_{2}$ and nonradioactive $\mathrm{SrF}_{2}$ are difficult. However the experimental data show the dissolution rate of ${ }^{90} \mathrm{SrF}_{2}$ in aqueous media is much greater than that of nonradioactive $\mathrm{SrF}_{2}$ of similar composition and thermal history. Results of a large number of tests with various grades of $\mathrm{SrF}_{2}$ show the average dissolution rate of $\mathrm{SrF}_{2}$ in demineralized water is about three times greater than in natural seawater. Although direct comparisons are difficult, $\mathrm{SrF}_{2}$ appears to dissolve at a more rapid rate than $\mathrm{SrTiO}_{3}$ and at a slower rate than $\mathrm{Sr}_{2} \mathrm{TiO}_{4}$.

Typical dissolution rate values for various grades of ${ }^{90} \mathrm{SrF}_{2}$ and nonradioactive $\mathrm{SrF}_{2}$ are given below: 
Dissolution Rate of Nonradioactive $\mathrm{SrF}_{2}$

in Aqueous Media at $24^{\circ} \mathrm{C}$

\begin{tabular}{|c|c|c|c|}
\hline \multirow[b]{2}{*}{$\begin{array}{c}\text { Grade of } \mathrm{SrF}_{2} \\
\text { Tested }\end{array}$} & \multirow[b]{2}{*}{$\begin{array}{c}\text { Surface Area } \\
\mathrm{cm}^{2} / \mathrm{g}\end{array}$} & \multicolumn{2}{|c|}{$\begin{array}{l}\text { Dissolution Rate } \\
\text { ug Sr Dissolved/hr-g Sr } \\
\text { Initially Present }\end{array}$} \\
\hline & & $\begin{array}{c}\begin{array}{c}\text { Demineralized } \\
\text { Water }\end{array} \\
\end{array}$ & $\begin{array}{l}\text { Naturat } \\
\text { Seawater } \\
\end{array}$ \\
\hline $\begin{array}{l}\text { Pure } \mathrm{SrF}_{2} \text { Fired at } \\
1100^{\circ} \mathrm{C} \text { for } 96 \mathrm{hr}\end{array}$ & 440 & $320 \pm 60$ & $120 \pm 36$ \\
\hline $\begin{array}{l}\text { Pure } \mathrm{SrF}_{2} \text { Fired at } \\
800^{\circ} \mathrm{C} \text { for } 240 \mathrm{hr}\end{array}$ & 510 & $660 \pm 140$ & $290 \pm 82$ \\
\hline $\begin{array}{l}\text { WESF-Grade } \mathrm{SrF}_{2} \\
\text { Fired at } 1100^{\circ} \mathrm{C} \\
\text { for } 1 \mathrm{hr}\end{array}$ & 440 & $130 \pm 24$ & $75 \pm 12$ \\
\hline $\begin{array}{l}\text { WESF-Grade } \mathrm{SrF}_{2} \\
\text { Fired at } 1100^{\circ} \mathrm{C} \text { for } \\
240 \mathrm{hr}\end{array}$ & 140 & $32 \pm$ & $24 \pm 6$ \\
\hline $\begin{array}{l}\text { WESF-Grade } \mathrm{SrF}_{2} \\
\text { Fired at } 800^{\circ} \mathrm{C} \text { for } \\
240 \mathrm{hr}\end{array}$ & 510 & $360 \pm 28$ & $130 \pm 12$ \\
\hline $\begin{array}{l}\text { WESF-Grade } \mathrm{SrF}_{2} \\
\text { Fired at } 800^{\circ} \mathrm{C} \text { for } \\
440 \mathrm{hr}\end{array}$ & 290 & $62 \pm$ & $36 \pm 4$ \\
\hline
\end{tabular}

Dissolution Rate of ${ }^{\circ} \mathrm{SrF}_{2}$ in Aqueous Media at $30^{\circ} \mathrm{C}$ (a) Dissolution Rate

Grade of ${ }^{9} \mathrm{SrF}_{2} \quad \mu \mathrm{g} \mathrm{Sr}$ Dissolved/hr-g Sr Initially Present Tested Demineralized Water Natura! Seawater

WESF-Produced
${ }^{\circ} \mathrm{SrF}_{2}$
$442 \pm 48$
$298 \pm 25$

WESF-Grade

${ }^{0} \mathrm{SrF}_{2}(\mathrm{~b}) \quad 1630 \pm 260 \quad 270 \pm 50$

(a) The surface area of ${ }^{\circ} \mathrm{SrF}_{2}$ could not be measured, and the dissolution rates are reported on the basis of contained strontium.

(b) Prepared by PNL using WESF flowsheet and ${ }^{\circ} \mathrm{Sr}$ feed solution from ARHCO. 
The Effect of Temperature on the Dissolution

Rate of Strontium Fluoride (a)

\begin{tabular}{|c|c|c|}
\hline \multirow{2}{*}{$\begin{array}{c}\text { Temperature } \\
{ }^{\circ} \mathrm{C}\end{array}$} & \multicolumn{2}{|c|}{$\begin{array}{l}\text { Dissolution Rate } \\
\mu \mathrm{g} \text { Sr Dissolved / hr-g Sr Initially Present }\end{array}$} \\
\hline & Demineralized Water & Natural Seawater \\
\hline 3 & 73 & 32 \\
\hline 9 & 76 & 44 \\
\hline 24 & 130 & 75 \\
\hline 34 & 250 & \\
\hline 35 & & 100 \\
\hline 42 & 300 & 130 \\
\hline 61 & 680 & \\
\hline 81 & 1900 & \\
\hline 90 & 2600 & \\
\hline
\end{tabular}

(a) WESF-Grade strontium fluoride fired at $1100^{\circ} \mathrm{C}$ for one $h$. Surface area $=440 \mathrm{~cm}^{2} / \mathrm{g}$.

\subsection{OPTICAL AND ELECTRONIC PROPERTIES}

Crystals having the fluorite structure, including strontium fluoride, have received a great deal of study in recent years in part because of their potential use in laser technology and superionic conduction. No attempt has been made to compile all of the published data on the optical and electronic properties of strontium fluoride. Some of the more pertinent data are given in the following sections.

\subsubsection{Infrared Spectrum (34)}

The infrared spectrum obtained with a single crystal of highpurity strontium fluoride is shown in Figure 15.

3.10.2 UPS and XPS Spectra (35)

Figure 16 shows the UPS and XPS spectra for single crystals of strontium fluoride.

\subsubsection{Reflectivity Spectrum}

Figure 17 shows the reflectivity spectrum for single crystals of strontium fluoride $(35,36)$. 


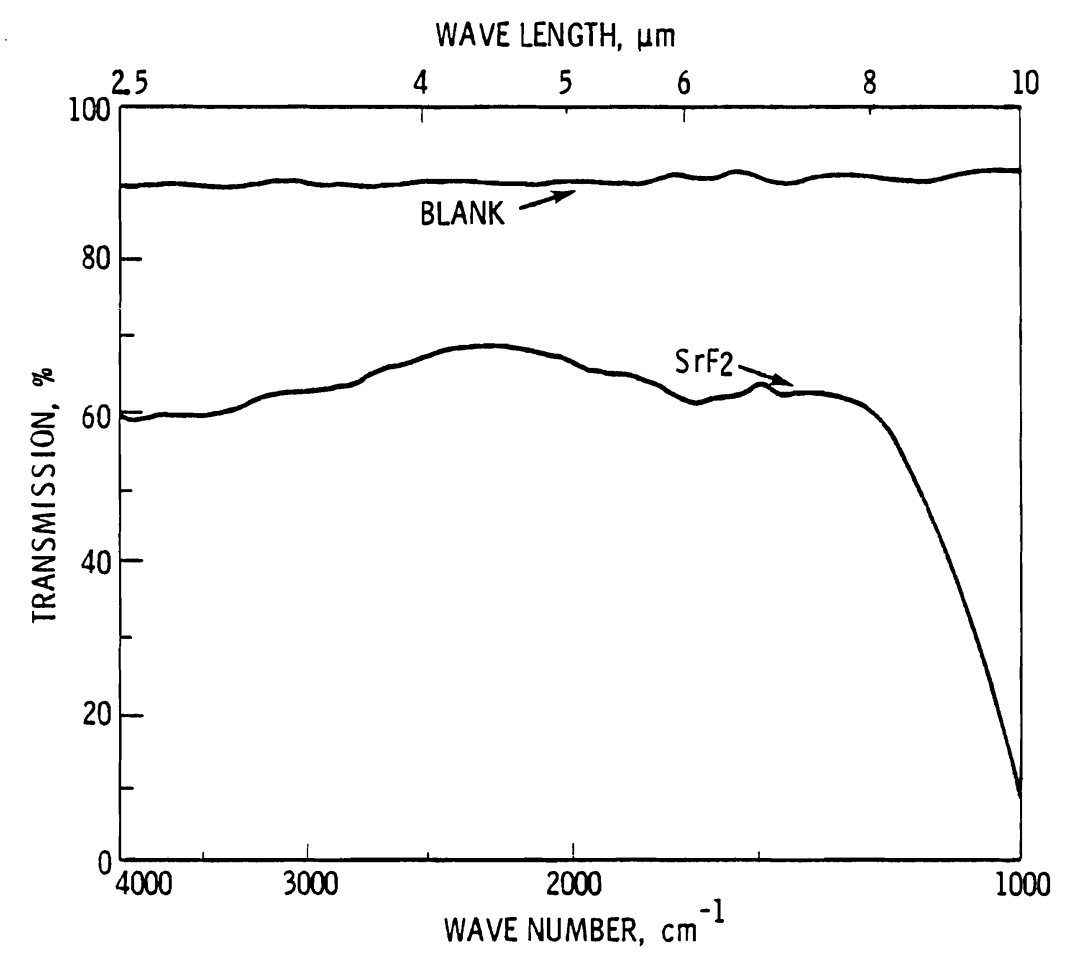

FIGURE 15. Infrared Transmission of a High Purity Strontium Fluoride Single Crystal (34).

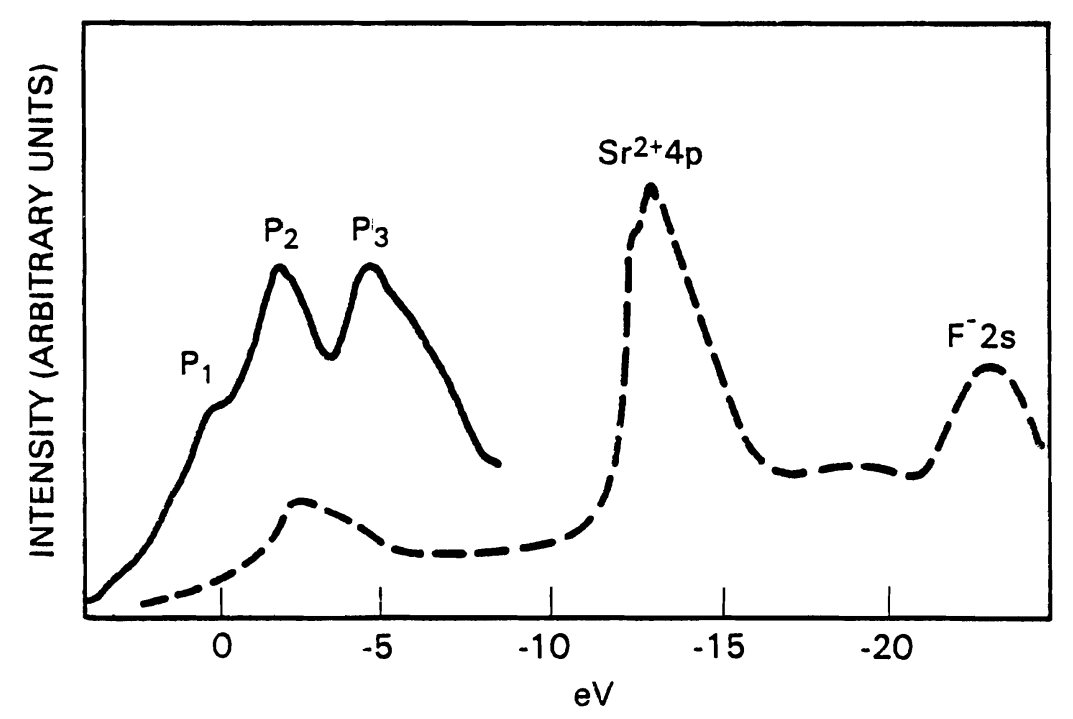

FIGURE 16. UPS (solid line) and XPS (dashed line) Spectra of Strontium Fluoride. (35) 


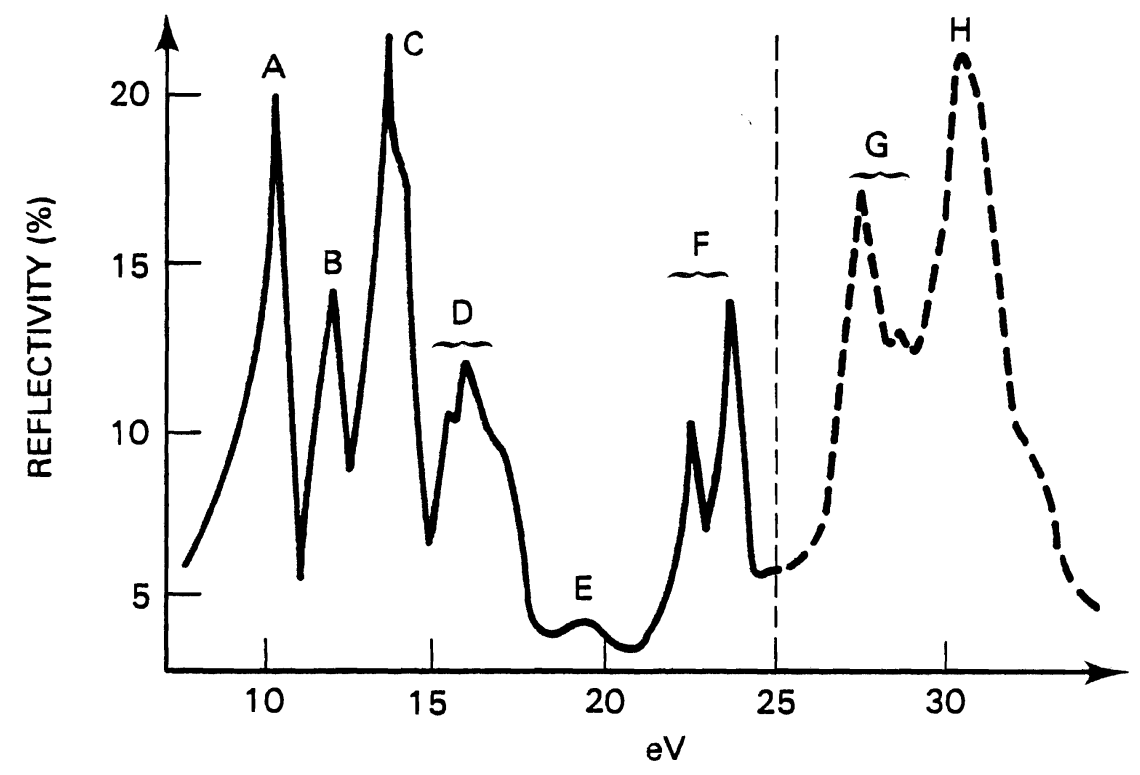

FIGURE 17. Refectivity of Strontium Fluoride [solid Tine-Raisin, et. al. (35), dashed line - Nisar and Robin (36)].

\subsubsection{Ionic Conductivity}

Strontium fluoride is an ionic conductor. Figure 18 shows the conductivity of pure crystalline and molten strontium fluoride as a function of temperature (37).

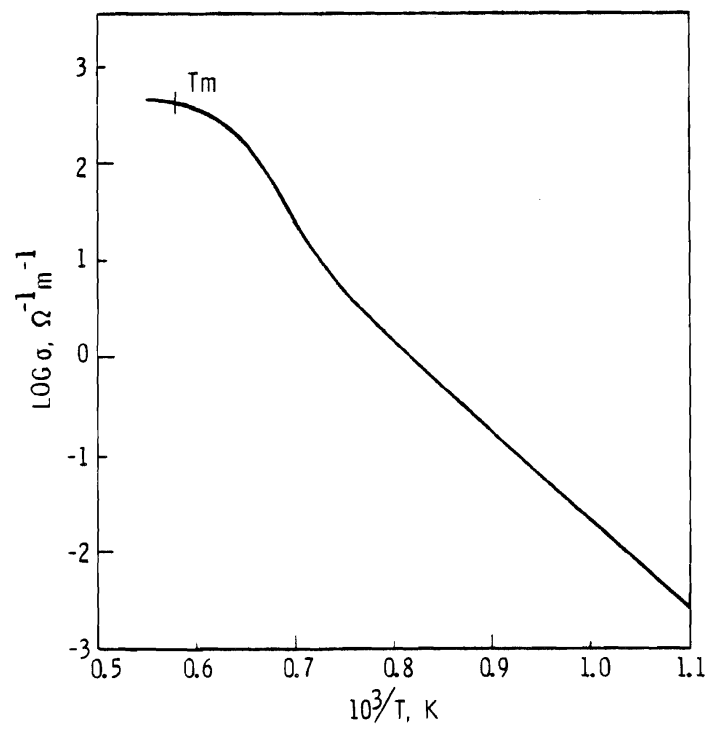

FIGURE 18. The Logarithm of the Conductivity $\sigma$ as a Function of the Reciprocal Temperature. Tm is the Melting Point. 
At high temperatures the conductivity is dependent on the frequency as shown in Figure 19 (38). Measuring frequencies greater than 100 $\mathrm{KHz}$ are required if the frequency dependent conductivity is to be avoided.

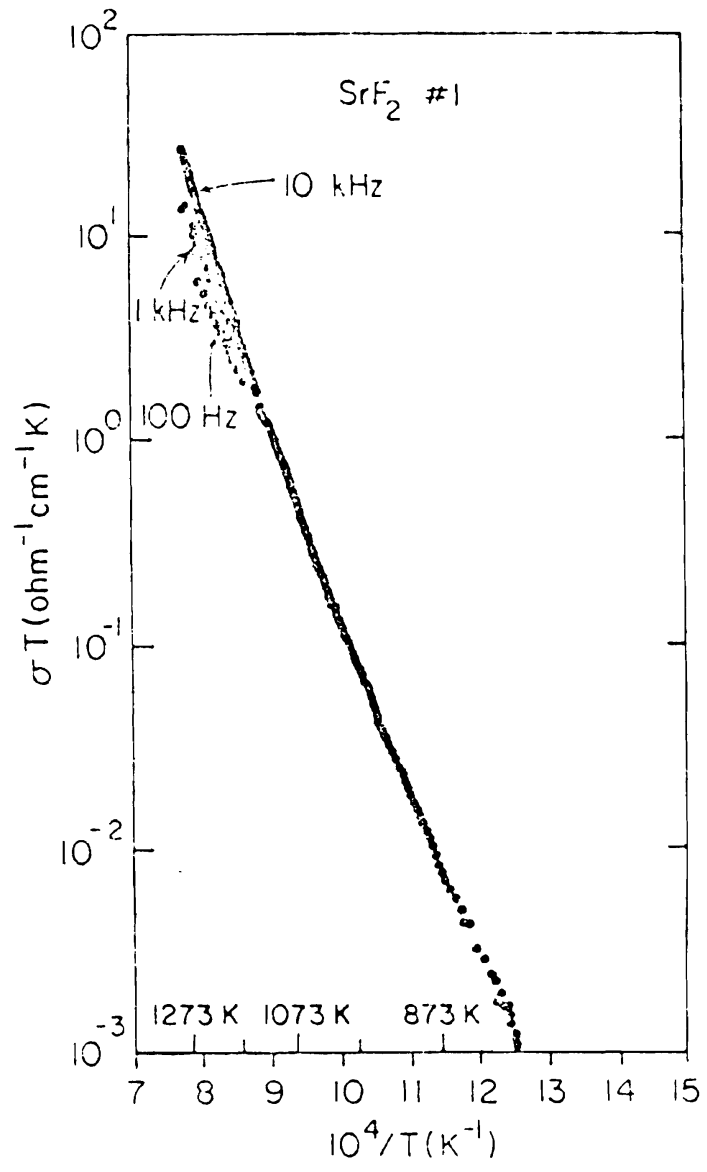

FIGURE 19. Frequency Dependence of the Conductivity $\sigma$ at High Temperatures.

The activation volumes of pure strontium fluoride were determined from the pressure dependence of the ionic conductivity (38). The values obtained are shown in Figure 20. 


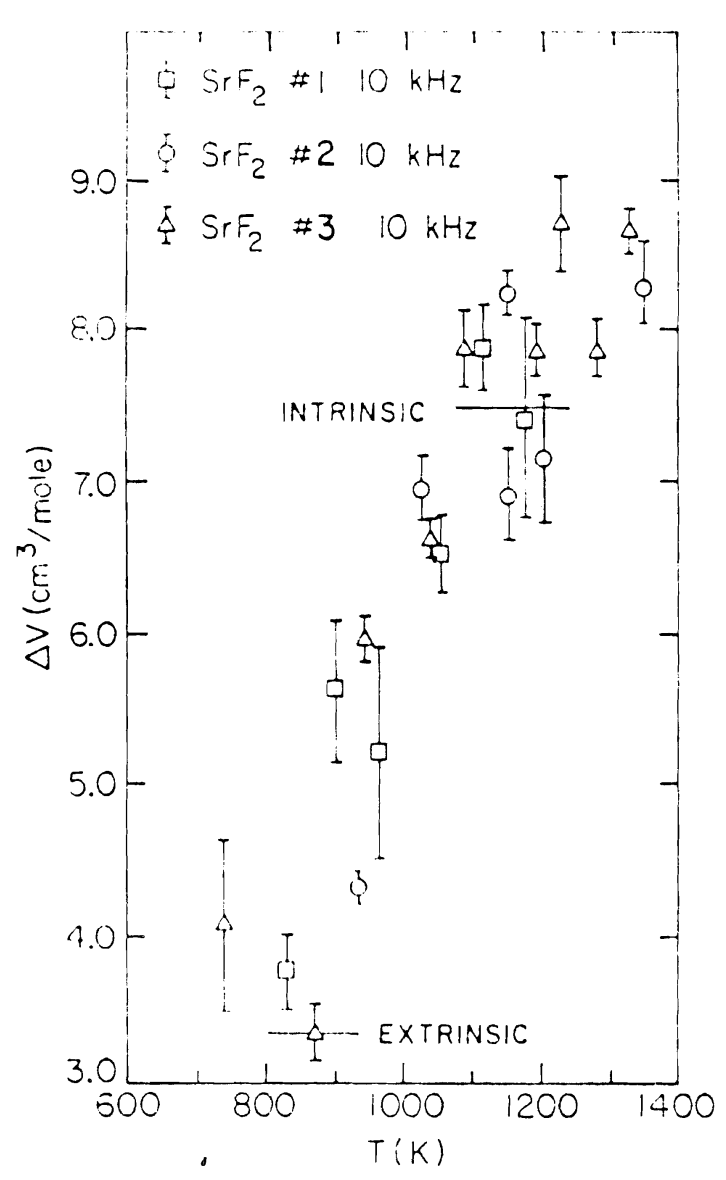

FIGURE 20. Activation Volumes For Pure Strontium Fluoride (38).

\subsubsection{Dielectric Constant (39)}

$$
\varepsilon_{S}^{S}\left(1000 \mathrm{~Hz}, 300^{\circ} \mathrm{K}\right)=6.47
$$




\subsection{MECHANICAL PROPERTIES}

3.11.1 Hardness (pure strontium fluoride)

Moh Hardness (30)

$$
H_{m}=3.5
$$

Knoop Hardness - Single Crystal

Unoriented Specimen (34)

$$
K_{\text {hn }}=140 \mathrm{~kg} / \mathrm{mm}^{2}
$$

Oriented Specimen (35)

Knoop Hardness Number in Several Crystallographic Directions for Strontium Fluoride (Values in parentheses are normalized with respect to $\langle 100\rangle$ )

\begin{tabular}{cc}
\hline Direction & Knoop Hardness Number \\
\cline { 2 - 3 } S1ip Mode & $\{100\}<110>$ \\
$<100>$ & $154(1.0)$ \\
$<210>$ & $143(0.93)$ \\
$<110>$ & $140(0.91)$ \\
$<211>$ & $135(0.87)$ \\
$<111>$ & $126(0.81)$
\end{tabular}

3.11.2 Modulus of Rupture (pure strontium fluoride) (41) 6100 psi 


\subsection{CHEMICAL PROPERTIES}

3.12.1 Thermodynamic Properties (21)

Selected Thermodynamic Functions for $\mathrm{SrF}_{2}$ - Crystal

\begin{tabular}{|c|c|c|c|c|c|}
\hline $\begin{array}{l}\text { Temperature } \\
{ }^{\circ} \mathrm{K} \\
\end{array}$ & $\begin{array}{l}S^{\circ} \\
\mathrm{eu}\end{array}$ & $\begin{array}{c}-\left(G^{0}-H_{208}\right) / T \\
\mathrm{Cal} / \mathrm{mol}-{ }^{\circ} \mathrm{K} \\
\end{array}$ & $\begin{array}{c}-\Delta H_{f}^{\circ} \\
\mathrm{kcal} / \mathrm{mol} \\
\end{array}$ & $\begin{array}{c}-\Delta G_{f^{\circ}} \\
\mathrm{kcal} / \mathrm{mol} \\
\end{array}$ & Log Kp \\
\hline 0 & 0 & Infinite & 290.4 & 290.4 & Infinite \\
\hline 100 & 5.02 & 32.91 & 291.1 & 286.9 & 627.0 \\
\hline 200 & 13.32 & 21.11 & 291.1 & 282.7 & 308.9 \\
\hline 298 & 19.63 & 19.63 & 290.9 & 278.6 & 204.2 \\
\hline 400 & 24.72 & 20.30 & 290.6 & 274.4 & 149.9 \\
\hline 500 & 28.77 & 21.60 & 290.3 & 270.4 & 118.2 \\
\hline 600 & 32.17 & 23.09 & 290.0 & 266.5 & 97.06 \\
\hline 700 & 35.10 & 24.60 & 289.7 & 262.6 & 81.98 \\
\hline 800 & 37.66 & 26.08 & 289.5 & 258.7 & 70.68 \\
\hline 900 & 39.96 & 27.49 & 289.5 & 254.9 & 61.89 \\
\hline 1000 & 42.07 & 28.85 & 289.3 & 251.0 & 54.86 \\
\hline 1100 & 44.07 & 30.14 & 290.9 & 247.1 & 49.09 \\
\hline 1200 & 46.01 & 31.38 & 290.4 & 243.1 & 44.28 \\
\hline 1300 & 47.96 & 32.58 & 289.7 & 239.2 & 40.21 \\
\hline 1400 & 50.13 & 33.76 & 288.6 & 235.4 & 36.74 \\
\hline 1500 & 52.80 & 34.94 & 286.4 & 231.6 & 33.75 \\
\hline 1600 & 54.92 & 36.12 & 284.9 & 228.0 & 31.15 \\
\hline 1700 & 56.65 & 37.28 & 316.4 & 223.6 & 28.75 \\
\hline 1800 & 58.15 & 38.40 & 315.2 & 218.2 & 26.49 \\
\hline 1900 & 59.51 & 39.47 & 314.1 & 212.8 & 24.48 \\
\hline 2000 & 60.75 & 40.51 & 313.1 & 207.5 & 22.68 \\
\hline 2100 & 61.90 & 41.50 & 312.2 & 202.3 & 21.05 \\
\hline 2200 & 62.98 & 42.45 & 311.3 & 197.0 & 19.58 \\
\hline
\end{tabular}


Selected Thermodynamic Functions for SrF 2 - Liquid (21)

\begin{tabular}{|c|c|c|c|c|c|}
\hline $\begin{array}{c}\text { Temperature } \\
{ }^{\circ} \mathrm{K} \\
\end{array}$ & $\begin{array}{c}\mathrm{S}^{\circ} \\
\mathrm{cal} / \mathrm{mol}-{ }^{\circ} \mathrm{K} \\
\end{array}$ & $\begin{array}{c}-\left(\mathrm{G}^{\circ}-\mathrm{H}_{298}^{\circ}\right) / \mathrm{T} \\
\mathrm{cal} / \mathrm{mol}-{ }^{\circ} \mathrm{K} \\
\end{array}$ & $\begin{array}{c}-\Delta H_{\mathrm{f}}^{\circ} \\
\mathrm{kcal} / \mathrm{mol} \\
\end{array}$ & $\begin{array}{c}-\Delta G_{\mathrm{f}}^{\circ} \\
\mathrm{kcal} / \mathrm{mol} \\
\end{array}$ & $\log k p$ \\
\hline & & $\therefore$ & & & \\
\hline 298 & 26.16 & 26.16 & 280.1 & 269.8 & 197.7 \\
\hline 400 & 31.25 & 26.83 & 279.8 & 266.3 & 145.5 \\
\hline 500 & 35.30 & 28.13 & 279.5 & 262.9 & 114.9 \\
\hline 600 & 38.70 & 29.63 & 279.2 & 259.6 & 94.6 \\
\hline 700 & 41.63 & 31.13 & 279.0 & 256.4 & 80.0 \\
\hline 800 & 44.19 & 32.61 & 278.7 & 253.2 & 69.2 \\
\hline 900 & 46.49 & 34.02 & 278.7 & 250.0 & 60.7 \\
\hline 1000 & 48.80 & 35.88 & 278.5 & 246.8 & 53.9 \\
\hline 1100 & 50.60 & 36.67 & 280.1 & 243.5 & 48.4 \\
\hline 1200 & 52.54 & 37.91 & 279.7 & 240.2 & 43.7 \\
\hline 1300 & 54.44 & 39.11 & 279.0 & 236.9 & 39.8 \\
\hline 1400 & 56.19 & 40.27 & 278.4 & 233.7 & 36.5 \\
\hline 1500 & 57.83 & 41.39 & 277.8 & 230.5 & 33.6 \\
\hline 1600 & 59.35 & 42.46 & 277.2 & 227.4 & 31.1 \\
\hline 1700 & 60.79 & 43.50 & 309.1 & 223.4 & 28.7 \\
\hline 1800 & 62.14 & 44.50 & 308.2 & 218.4 & 26.5 \\
\hline 1900 & 63.42 & 45.46 & 307.3 & 213.4 & 24.5 \\
\hline 2000 & 64.64 & 46.39 & 306.3 & 208.5 & 22.8 \\
\hline 2200 & 66.89 & 48.15 & 304.5 & 198.8 & 19.8 \\
\hline 2400 & 68.95 & 49.80 & 302.6 & 189.3 & 17.2 \\
\hline 2600 & 70.85 & 51.35 & 300.9 & 179.9 & 15.1 \\
\hline 2800 & 72.60 & 52.80 & 299.1 & 170.7 & 13.3 \\
\hline 3000 & 74.23 & 54.18 & 297.5 & 161.6 & 11.8 \\
\hline 3200 & 75.76 & 55.48 & 296.0 & 152.6 & 10.4 \\
\hline 3400 & 77.20 & 56.72 & 294.6 & 143.6 & 5.2 \\
\hline
\end{tabular}


Selected Thermodynamic Properties for SrF2-Ideal Gas (21)

\begin{tabular}{|c|c|c|c|c|c|}
\hline $\begin{array}{l}\text { Temperature } \\
{ }^{\circ} \mathrm{K} \\
\end{array}$ & $\begin{array}{c}\mathrm{S}^{\circ} \\
\mathrm{cal} / \mathrm{mol}-{ }^{\circ} \mathrm{K} \\
\end{array}$ & $\begin{array}{c}-\left(G^{\circ}-H^{\circ} 298\right) / T \\
\mathrm{cal} / \mathrm{mol}-{ }^{\circ} \mathrm{K}\end{array}$ & $\begin{array}{c}-\Delta \mathrm{H}_{\mathrm{f}}^{\circ} \\
\mathrm{kcal} / \mathrm{mol}\end{array}$ & $\begin{array}{c}-\Delta G_{f}^{\circ} \\
\mathrm{kcal} / \mathrm{mol}\end{array}$ & Log K \\
\hline 0 & 0 & Infinite & 182.7 & 182.7 & Infinite \\
\hline 100 & 57.35 & 80.24 & 182.8 & 183.8 & 401.8 \\
\hline 200 & 64.82 & 70.83 & 183.0 & 184.8 & 202.0 \\
\hline 298 & 69.69 & 69.69 & 183.1 & 185.7 & 136.1 \\
\hline 400 & 73.49 & 70.20 & 183.2 & 186.6 & 101.9 \\
\hline 500 & 76.46 & 71.16 & 183.4 & 187.4 & 81.9 \\
\hline 600 & 78.92 & 72.26 & 183.6 & 188.2 & 68.5 \\
\hline 700 & 81.01 & 73.36 & 183.9 & 188.9 & 56.0 \\
\hline 800 & 82.84 & 74.43 & 184.3 & 189.6 & 51.8 \\
\hline 900 & 84.45 & 75.46 & 184.9 & 190.2 & 46.2 \\
\hline 1000 & 85.90 & 76.43 & 185.3 & 190.8 & 41.7 \\
\hline 1100 & 87.22 & 77.35 & 187.6 & 191.2 & 38.0 \\
\hline 1200 & 88.42 & 78.23 & 188.0 & 191.5 & 34.9 \\
\hline 1300 & 89.53 & 79.05 & 188.3 & 191.8 & 32.2 \\
\hline 1400 & 90.55 & 79.84 & 188.7 & 192.1 & 30.0 \\
\hline 1500 & 91.51 & 80.59 & 189.0 & 192.3 & 28.0 \\
\hline 1600 & 92.40 & 81.30 & 189.4 & 192.5 & 26.3 \\
\hline 1700 & 93.24 & 81.98 & 222.4 & 191.8 & 24.7 \\
\hline 1800 & 94.03 & 82.62 & 222.4 & 190.0 & 23.1 \\
\hline 1900 & 94.78 & 83.24 & 222.4 & 188.2 & 21.6 \\
\hline 2000 & 95.50 & 83.84 & 222.5 & 186.4 & 20.4 \\
\hline 2200 & 96.82 & 84.96 & 222.6 & 182.8 & 18.2 \\
\hline 2400 & 98.03 & 86.00 & 222.7 & 179.1 & 16.3 \\
\hline 2600 & 99.14 & 86.97 & 222.9 & 175.5 & 14.8 \\
\hline 2800 & 100.17 & 87.87 & 223.1 & 172.8 & 13.4 \\
\hline 3000 & 101.13 & 88.73 & 223.4 & 168.2 & 12.3 \\
\hline 3500 & 103.27 & 90.65 & 224.7 & 158.9 & 9.9 \\
\hline 4000 & 105.12 & 92.35 & 227.0 & 149.3 & 8.2 \\
\hline 4500 & 106.76 & 93.86 & 230.3 & 139.4 & 6.8 \\
\hline 5000 & 108.23 & 95.23 & 234.7 & 129.1 & 5.6 \\
\hline 5500 & 109.55 & 96.47 & 239.9 & 118.3 & 4.7 \\
\hline 6000 & 110.76 & 97.61 & 245.7 & 107.0 & 3.9 \\
\hline
\end{tabular}




\subsubsection{Chemical Reactions}

a. Oxygen - Does not react to any appreciable degree at temperatures below $1000^{\circ} \mathrm{C}$.

- Reacts with the surface of strontium fluoride single crystals at elevated temperatures to give highly conductive coating $(42,43)$.

b. Nitrogen - No reaction

c. Water - Strontium fluoride is slightly soluble (see Sections 3.9.16 and 3.9.17).

- Water vapor reacts with the surface of strontium fluoride single crystals to give a highly conductive coating $(42,43)$

d. Hydrochloric Acid - Dissolves in boiling $6 \mathrm{~N} \mathrm{HCl}$

e. Nitric Acid - Dissolves in hot 2-6N $\mathrm{HNO}_{3}$

f. Sulfuric Acid - Reacts with hot concentrated $\mathrm{H}_{2} \mathrm{SO}_{4}$

g. Containment materials - See Section 3.15.

\subsection{COMPACTION CHARACTERISTICS}

\subsection{3 .1 Cold Pressing (44)}

Strontium fluoride is readily compacted to moderately high densities using cold pressing techniques. RHO uses pneumatic impaction, which is essentially a cold pressing operation, to obtain a strontium fluoride density of $70 \%-75 \%$ of theoretical density in the WESF storage capsule.

Figure 21 shows the densities obtained when three grades of strontium fluoride were cold pressed into cylindrical pellets having a length-to-diameter ratio of approximately 0.5 .

The temperature at which the strontium fluoride is fired affects the density which can be obtained by cold pressing as shown in Figure 22. 


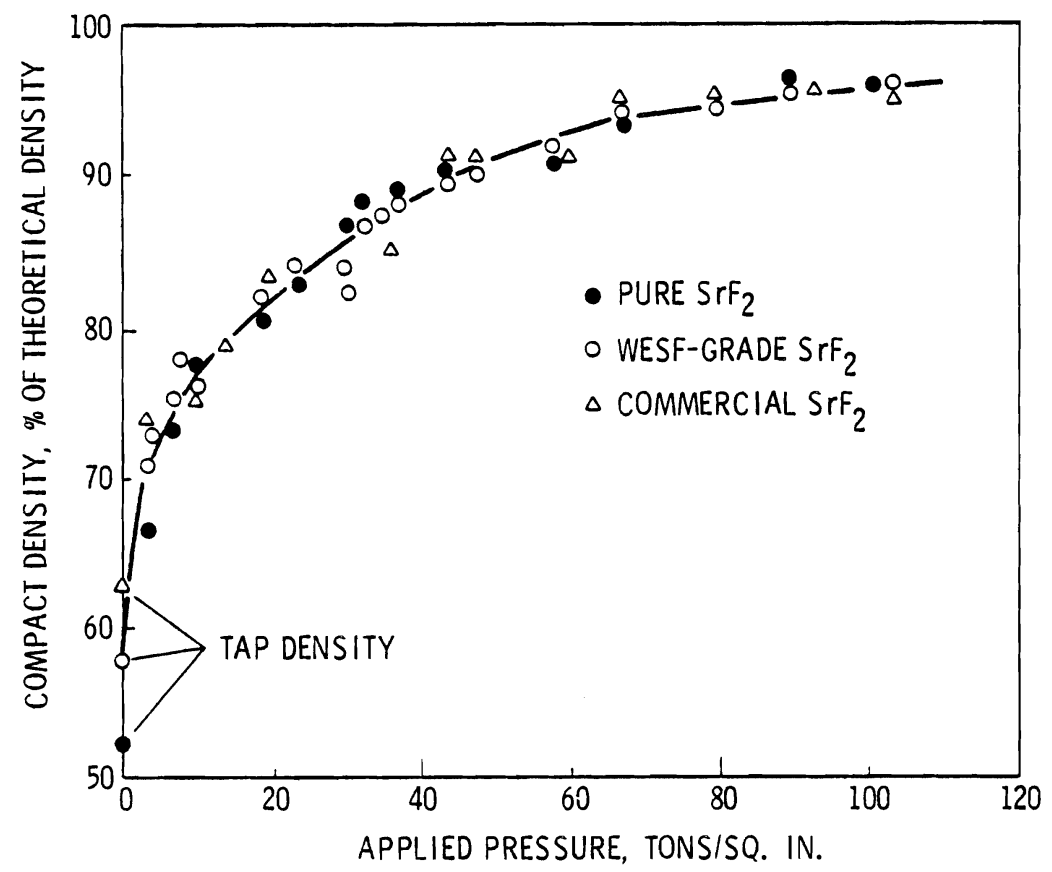

FIGURE 21. Density of Cold Pressed Pellets As a Function of Applied Pressure for Three Grades of Strontium Fluoride Which Had Been Fired at $1100^{\circ} \mathrm{C}$ for 16 Hours and Crushed to a -20 Mesh Powder.

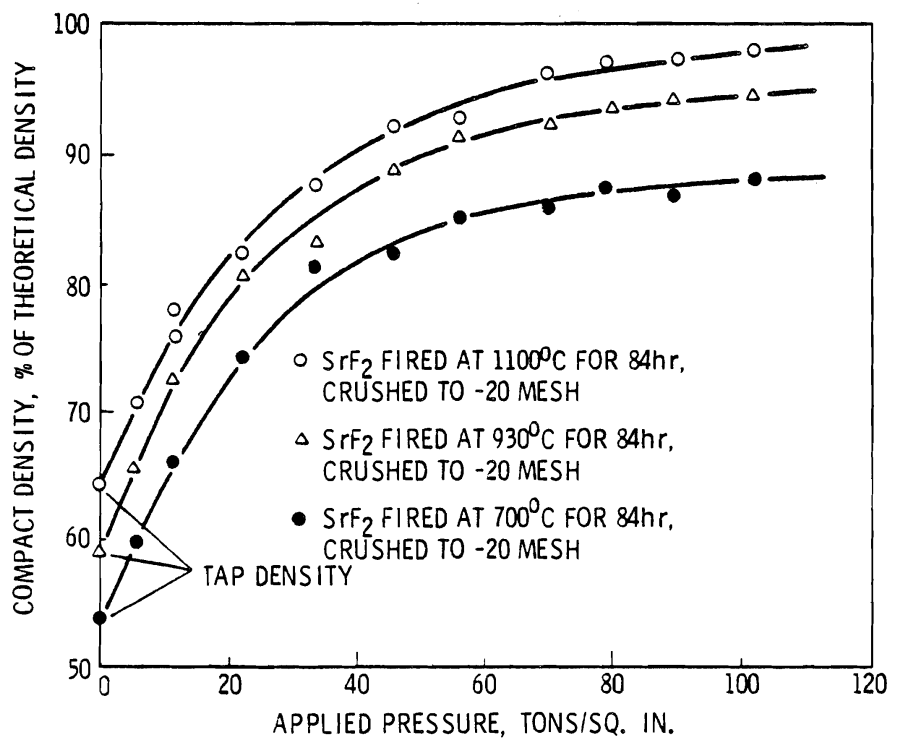

FIGURE 22. The Effect of Firing Temperature on the Cold Pressing of Commercial Strontium Fluoride. 
The particle size of the strontium fluoride appears to have only a minor effect on the density that can be obtained by cold pressing. The following table shows results obtained using commercial grade strontium fluoride of various mesh sizes.

The Effect of Particle Size on the Cold Pressing of Commercial $\mathrm{SrF}_{2}$ Fired at $1100^{\circ} \mathrm{C}$ for 84 Hours and Crushed

Size Fraction

Compacted Density, $\%$ of theoretical density (U.S. Standard Sieve) 11.1 tons/sq in. 34.0 tons $/ \mathrm{sq}$ in.

$-20+70$ 78

$-70+100$ 78 89

$-100+140$

80

89

$-140+200$

78

87

$-200+230$

75

87

$-230+325$

76

86

$-325$

75

85

Combined Fractions

78

\subsubsection{Hot Pressing (45)}

WESF-grade strontium fluoride was successfully hot-pressed in a graphite die at temperatures up to $825^{\circ} \mathrm{C}$ and pressures up to 4950 psi. Figure 23 shows the pellet densities obtained as a function of temperature and applied pressure. Pellet densities exceeding $98 \%$ of theoretical density were obtained at $825^{\circ} \mathrm{C}$-the theoretical density of WESF grade strontium fluoride is calculated to be $4.24 \mathrm{~g} / \mathrm{cm}^{3}$ (See Section 3.9.1). The graphite die had an inner diameter of one inch, and sufficient strontium fluoride was used for each pressing to give a compacted pellet having a lengthto-diameter ratio (L/D) of approximately one. The compacted pellets were free of visible flaws and cracks, but were covered with a thin coating of graphite. The graphite layer was easily removed by heating the pellets in air at $800^{\circ} \mathrm{C}$ for 30 minutes. Thermal stability of the pellets was very good, and they could be placed directly into or removed from an $800{ }^{\circ} \mathrm{C}$ furnace without fracturing or cracking. 
Attempts to hot press the WESF-grade strontium fluoride at $850^{\circ} \mathrm{C}$ and higher were unsuccessful because the fluoride adhered to the graphire die.

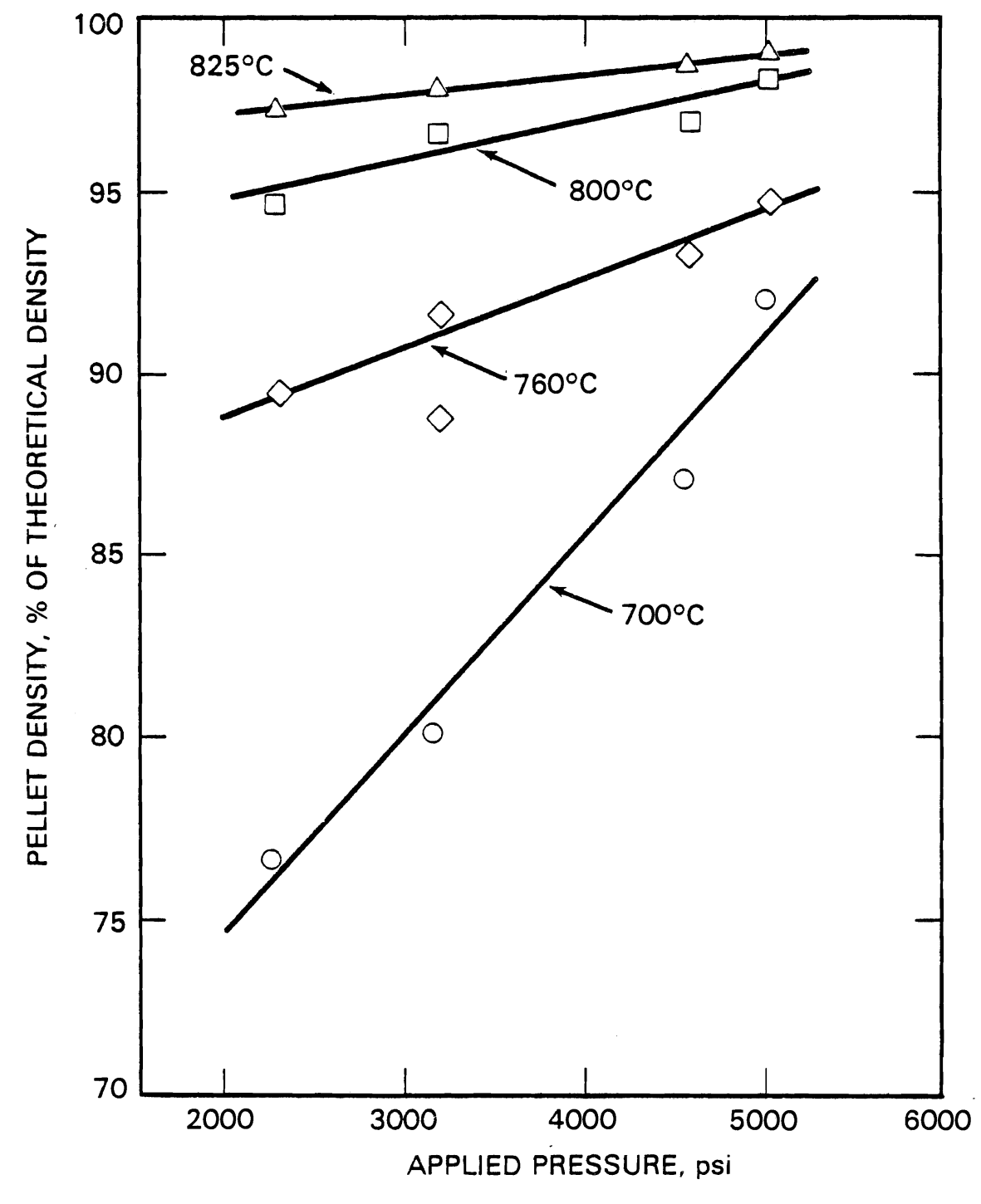

FIGURE 23. The Effect of Temperature and Applied Pressure on the Density of Hot-Pressed Pellets of WESF-Grade Strontium Fluoride (45). 


\subsection{SINTERING CHARACTERISTICS $(33)$}

Because of its relatively low melting point, strontium fluoride compacted to less than theoretical density will sinter and agglomerate when held at the temperatures expected in an operating ${ }^{90} \mathrm{SrF}_{2}$ heat source. The presence of impurities in the $\mathrm{SrF}_{2}$, which can result in the formation of low melting phases (see Section 3.9.6) will increase the tendency for agglomeration to occur. How the surface area of freshly prepared $\mathrm{SrF}_{2}$ decreases when the $\mathrm{SrF}_{2}$ is fired at various temperatures for extended periods of time is shown in the table below.

Since the standard WESF ${ }^{9} \mathrm{SrF}_{2}$ capsule contains strontium fluoride compacted to approximately $70 \%$ of theoretical density, additional sintering and agglomeration of the $\mathrm{SrF}_{2}$ could occur during heat source service. This could produce changes in the distribution of the $\mathrm{SrF}_{2}$ in the capsule during use and possibly affect: (a) ${ }^{90} \mathrm{SrF}_{2}$-clad compatibitity and, (b) the heat flow pattern in the capsule.

\begin{tabular}{|c|c|c|c|c|c|c|}
\hline \multirow{3}{*}{$\begin{array}{l}\text { Time At } \\
\text { Temp. } \\
\text { (hrs) } \\
\end{array}$} & \multicolumn{6}{|c|}{ Surface Area, $\mathrm{cm}^{2} / \mathrm{g}$ of $\mathrm{SrF}_{2}$} \\
\hline & \multicolumn{2}{|r|}{$600^{\circ} \mathrm{C}$} & \multicolumn{2}{|c|}{$800^{\circ} \mathrm{C}$} & \multicolumn{2}{|c|}{$1100^{\circ} \mathrm{C}$} \\
\hline & $\begin{array}{l}\text { Pure } \\
\text { SrF } 2 \\
\end{array}$ & $\begin{array}{c}\text { WESF-Grade } \\
\mathrm{SrF}_{2} \\
\end{array}$ & $\begin{array}{l}\text { Pure } \\
\text { SrF }_{2} \\
\end{array}$ & $\begin{array}{l}\text { WESF-Grade } \\
\mathrm{SrF}_{2} \\
\end{array}$ & $\begin{array}{l}\text { Pure } \\
\text { SrF } 2 \\
\end{array}$ & $\begin{array}{l}\text { WESF-Grade } \\
\mathrm{SrF}_{2} \\
\end{array}$ \\
\hline 0 & 2550 & 2700 & 2550 & 2700 & 2550 & 2700 \\
\hline 0.5 & & 2280 & & 820 & & 380 \\
\hline 1 & 2420 & 2000 & 1250 & 970 & 860 & 460 \\
\hline 2 & 2320 & 1850 & 1210 & & 810 & 520 \\
\hline 4 & 2130 & 1740 & 1150 & 1030 & 730 & 380 \\
\hline 8 & 1950 & 1650 & 1080 & 950 & 650 & 380 \\
\hline 24 & 1660 & 1530 & 920 & 830 & 520 & 370 \\
\hline 48 & 1650 & & & & & \\
\hline 72 & & 1410 & & 540 & & 230 \\
\hline 96 & 1530 & & 680 & & 420 & \\
\hline 240 & 1490 & 1230 & 510 & 510 & 390 & 140 \\
\hline 480 & 1450 & & & & & \\
\hline 1500 & 1470 & & 540 & 470 & 360 & 130 \\
\hline 4400 & & & & 480 & & \\
\hline
\end{tabular}




\subsection{COMPATIBILITY WITH CONTAINMENT MATERIALS}

Extensive studies were carried out at PNL to evalute the compatibility of nonradioactive $\mathrm{SrF}_{2},{ }^{90} \mathrm{SrF}_{2}$ and WESF-produced ${ }^{90} \mathrm{SrF}_{2}$ with metallic containment materials at temperatures of $600^{\circ}$ to $1100^{\circ} \mathrm{C}$. (Details of the various compatibility studies are presented in References 7, 46-48). The results obtained show that almost all of the metals tested suffered extensive attack when exposed to the strontium fluoride at elevated temperatures. As a general rule, the precious metals and refractory metals were more resistant to fluoride attack than the base metals and transition metals. Exceptions to the rule were platinum and gold which suffered catastrophic attack.

Most of the metals exposed to strontium fluoride suffered two distinctly different types of attack which have been designated as (a) chemical attack and (b) microstructural changes.

- Chemical attack is metal attack which can be directly attributed to reaction of the metal with components of the strontium fluoride. It includes the dissolution of the metal, leaching of alloy components, intergranular (grain boundary) attack, pitting, subsurface void formation, etc.

- Microstructural changes are those instances where the metal exhibits areas in which the morphology differs from that of the normal metal morphology. The microstructural changes usually consist of one or more of the following: (a) the disappearance of normal alloy precipitates, (b) the formation of abnormal precipitates, and (c) marked changes in grain size. They do include the normal changes in morphology which occur when metals are aged at elevated temperatures for extended periods of time.

From a safety standpoint, chemical attack is probably a more serious problem than microstructural changes. Carried to the extreme, chemical attack can result in the breaching of the containment vessel and a subsequent release of radioactivity. The chemical attack may also result in a marked decrease in the structural strength of the containment vessel. The microstructural changes, while they may affect the structural strength of the containment vessel, would not result directly in a breach of the containment.

All of the compatibility data show that attack of the various metals is due primarily to impurities in the strontium fluoride. Once the critical impurities in the strontium fluoride have reacted with the metal, the rate of metal attack decreases to a very low level. In general, metal specimens exposed to WESF produced ${ }^{90} \mathrm{SrF}_{2}$ for 20,000 and $30,000 \mathrm{~h}$ suffered no greater attack than specimens tested for 6000 and 12,000 h. The test data show that fluorides of iron and chromium in the strontium fluoride are the prime contributors to the metal attack. If SrO is present in the SrF2, it also causes extensive metal attack. Fortunately, the data indicate that the Sro concentration in SrF 2 is very low. 
Results of some of the compatibility tests are summarized in the following tables. The complete results are given in the reports referenced.

Two full-size WESF storage capsules containing strontium-90 fluoride were allowed to self-heat in insulated containers to approximately $800^{\circ} \mathrm{C}$. Because of uneven heat losses from the containers, a temperature gradient existed between the midpoint and ends of each capsule. One capsule was held at temperature for $5000 \mathrm{~h}$, while the second was held at temperature for $26,200 \mathrm{~h}$. The capsules were sectioned and samples of the Hastelloy C-276 from the inner capsules, which was in contact with the strontium-90 fluoride, were taken at various locations. The metal attack and approximate metal/ fluoride interface temperature at each location are given in the following table.

Attack of Hastelloy C-276 Exposed To WESF-Produced Strontium-90 Fluoride (48)

Test Duration, h

$\frac{h}{5,000} \frac{\text { Section }}{3}$

26,200
Interface

Temperature, ${ }^{\circ} \mathrm{C}$

762
821
813
790
778
831
824
800

Depth of Hastelloy C-276 Affected (a), $\mu m$ Chemical Microstructural Attack Changes

$\begin{array}{rr}80 & 0 \\ 230 & 0 \\ 210 & 0 \\ 280 & 0 \\ & \\ 130 & 0 \\ 180 & 310 \\ 310 & 560 \\ 230 & 510\end{array}$

(a) Estimated from photomicrographs of the affected areas

Hastelloy C-276, Haynes Alloy 25, and TZM were tested with WESF-Produced strontium-90 fluoride and inert strontium fluoride at $600^{\circ}$ to $1000^{\circ} \mathrm{C}$ for up to $30,000 \mathrm{~h}$. The results obtained with the strontium-90 fluoride are summarized in the table on page 59 .

Thirty three metal were tested with inert strontium fluoride at $800^{\circ} \mathrm{C}$ for 1500 and $4400 \mathrm{~h}$. The strontium fluoride contained impurities similar to those found in WESF-produced strontium-90 fluoride. The results obtained are presented in the table on page 60. 


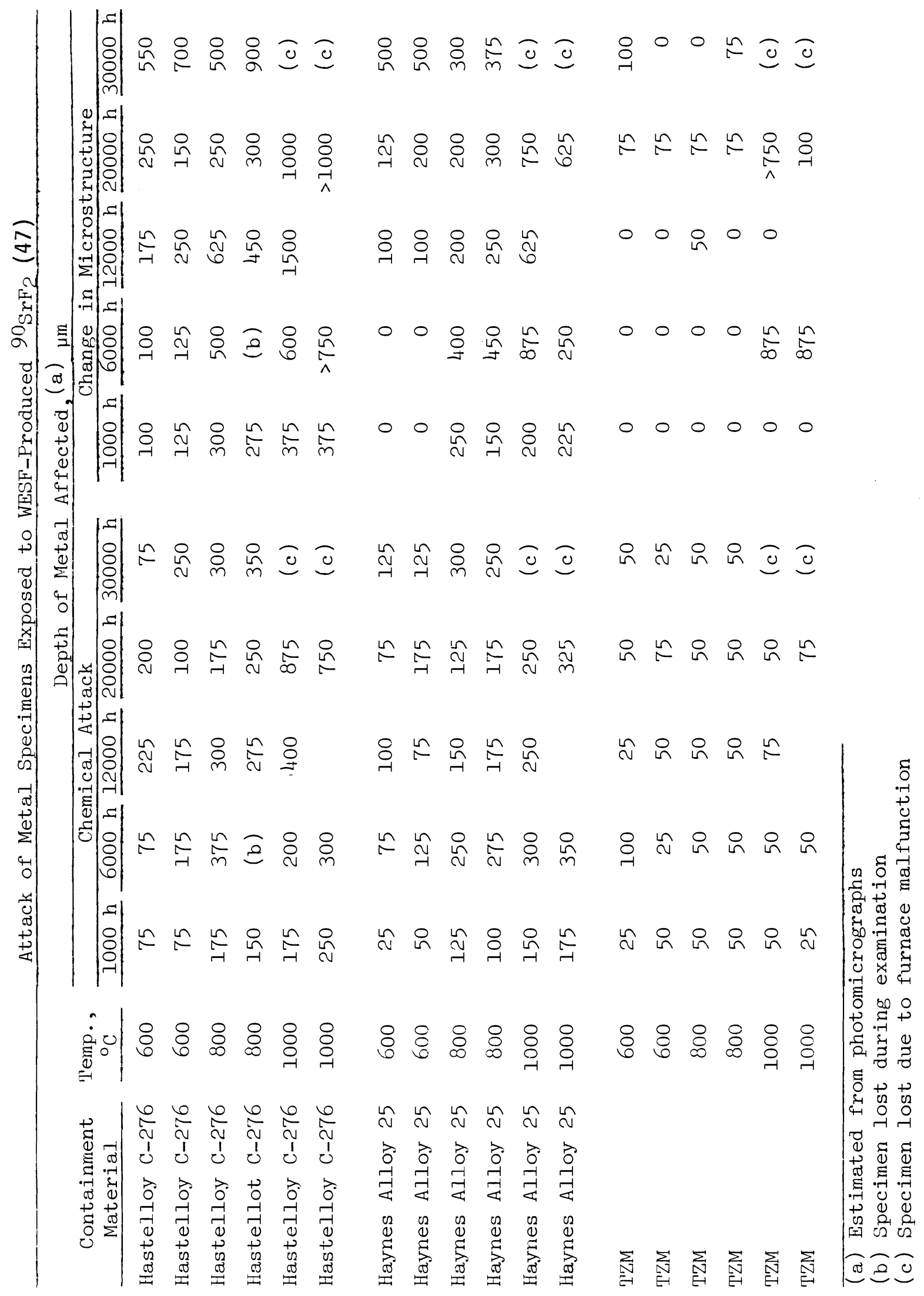


Attack of Metals Exposed to Inert Strontium Fluoride(a) at $800^{\circ} \mathrm{C}(46)$ Depth of Metal Affected, $\mu \mathrm{m}$

\begin{tabular}{|c|c|c|c|c|}
\hline \multirow[b]{2}{*}{ Containment Material } & \multicolumn{2}{|c|}{ Chemical Attack } & \multicolumn{2}{|c|}{$\begin{array}{c}\text { Changes in } \\
\text { Microstructure }\end{array}$} \\
\hline & $1500 \mathrm{~h}$ & $4400 \mathrm{~h}$ & $1500 \mathrm{~h}$ & $4400 \mathrm{~h}$ \\
\hline Hastelloy C-276 & 75 & 75 & 175 & 125 \\
\hline Haynes Alloy 25 & 50 & 25 & 75 & 50 \\
\hline TZM & $<25$ & $<25$ & 0 & $<25$ \\
\hline Hastelloy $\mathrm{C}-4$ & 125 & 100 & 300 & 325 \\
\hline Hastelloy S & 175 & 75 & 375 & 300 \\
\hline Hastelloy B & 100 & 100 & 375 & 275 \\
\hline Hastelloy B-2 & 250 & 150 & 450 & 450 \\
\hline Haynes Alloy 556 & 125 & 125 & 150 & 175 \\
\hline Inconel 617 & 175 & 200 & 350 & 350 \\
\hline Inconel 671 & 625 & 500 & 625 & 500 \\
\hline Incoloy 800 & 200 & 175 & 0 & 0 \\
\hline Rene 41 & 250 & 275 & 350 & 325 \\
\hline Udimet 700 & $>625$ & $>625$ & 0 & $>625$ \\
\hline Monel 400 & 125 & 150 & 200 & 150 \\
\hline Nickel 200 & 175 & 200 & 250 & 200 \\
\hline Ingot Iron & 75 & 100 & 0 & 175 \\
\hline $\begin{array}{l}\text { Ductile Cast Iron } \\
\text { (Pearlite Anneal) }\end{array}$ & 75 & 100 & 50 & 125 \\
\hline $\begin{array}{l}\text { Ductile Cast Iron } \\
\text { (Ferrite Anneal) }\end{array}$ & 75 & 100 & 50 & 175 \\
\hline 316I Stainless Steel & 150 & 550 & 0 & 0 \\
\hline JS 777 & 150 & 125 & 175 & 150 \\
\hline Copper & $>625$ & $>625$ & 0 & 0 \\
\hline Titanium & $>625$ & $>625$ & $X(c)$ & $\mathrm{X}$ \\
\hline Hafnalloy 2525 (d) & $>625$ & $\mathrm{CR}(\mathrm{b})$ & $\mathrm{X}$ & $\mathrm{X}$ \\
\hline Molybdenum & 50 & $<25$ & 0 & $<25$ \\
\hline Niobium & 75 & 50 & 50 & 50 \\
\hline $\mathrm{Ta}-10 \% \mathrm{~W}$ & 250 & 50 & 0 & 0 \\
\hline Mo-50\% Re & 50 & 25 & 0 & 0 \\
\hline $\mathrm{W}-26 \% \mathrm{Re}$ & 50 & 25 & 0 & 0 \\
\hline Rhenium & $<25$ & $<25$ & 0 & 0 \\
\hline Iridium & 0 & $<<25$ & 0 & 0 \\
\hline $\mathrm{Ir}-0.3 \% \mathrm{~W}$ & 0 & $<<25$ & 0 & 0 \\
\hline Platinum & $>625$ & $>625$ & $\mathrm{X}$ & $\mathrm{X}$ \\
\hline Gold & $>375$ & $>375$ & $\ddot{X}$ & $X$ \\
\hline
\end{tabular}

(a) The strontium fluoride contained impurities similar to those found in WESF-produced strontium-90 fluoride

(b) CR - Complete reaction

(c) X - Could not be estimated because of extensive chemical attack

(d) Hafnalloy 2525 is a hafnium based alloy containing $0.25 \% \mathrm{Pt}$ and $0.25 \% \mathrm{Pd}$ 


\subsection{REFERENCES}

1. W. P. Bebbington, Reprocessing of Nuclear Fuel, Scientific American, $238(6)$ : 34-41, December 1976.

2. H. T. Fullam, Strontium-90 Fluoride Data Sheet, BNWL-2284, Pacific Northwest Laboratory, Richland, WA, 1977.

3. S. J. Rimshaw and E. E. Ketchen, Strontium-90 Data Sheets, ORNL4358, Oak Ridge National Laboratory, Oak Ridge, TN, 1969.

4. D. C. Kocher, Nuclear Data Sheets 16 (1):55-107, 1975.

5. N. E. Holden and F. W. Walker, Chart of the Nuclides, 11th Ed., Knolls Atomic Power Laboratory, General Electric Co., Schenectady, NY, 1972.

6. H. H. Van Tuyl, Pacific Northwest Laboratory, unpublished data.

7. H. T. Fullam, The Containment of $90 \mathrm{SrF}_{2}$ at 800 to $1100^{\circ} \mathrm{C}$, Preliminary Results, BNWL-1967, Pacific Northwest Laboratory, Richland, WA, 1976.

8. H. T. Fullam, Quarterly Report on the Strontium Heat Source Development Program and Beneficial Isotopes Utilization Program, Division of Nuclear Research and Applications for July-September 1977, BNWL-1845-36, Pacific Northwest Laboratory, Richland, WA, 1977.

9. F. K. McTaggart and A. G. Turnbu11, Aust. J. Chem. 17:727-30, 1964.

10. C. M. Lederer, J. M. Hollander, and I. Perlman, Table of Isotopes, John Wiley and Sons, Inc., New York, NY, 1967.

11. C. A. Rohrmann, Radioisotope Heat Sources, HW-76323-Rev. 1, General Electric Co., Richland, WA 1963.

12. E. D. Arnold, Handbook of Shielding Requirements and Radiation Characteristics of Radioisotope Power Sources for Terrestrial, Marine and Space Applications, ORNL-3576, Oak Ridge National Laboratory, Oak Ridge, TN, 1964.

13. B. S. Dzhelepov and L. L. Peker, Decay Schemes of Radioactive Nuclei, Pergamon Press, New York, NY, 1961.

14. Recommendations of the International Commission on Radiological Protection, Report of Committee II on Permissible Dose for Internal Radiation, ICRP Publication 2, Radiation Protection, published for ICRP by Pergamon Press, New York, NY, 1959. 
15. Recommendations of the International Commission on Radiological Protection, Revised 1962, ICRP Publication 6, Radiation Protection, published for ICRP by Pergamon Press, New York, NY, 1962.

16. Code of Federal Regulations, Title 10 - Energy, Part 20, 1976.

17. United States Energy Research and Development Administration Manual Chapter-0524, Standards for Radiation Protection, March 1977.

18. V. Croatto and M. Bruno, Gazz.Chem. Ital. 78:95-105, 1948.

19. A. D. Kirshenbaum, J. A. Cahill, and C. J. Stokes, J. Inorg. Nucl. Chem. , 15:297-304, 1960 .

20. G. B. Mellinger and J. L. Bates, Pacific Northwest Laboratory Quarterly Report to Advanced Nuclear Energy Systems, Space and Special purposes Division for April-June 1975, BNWL-2845-13, Pacific Northwest Laboratory, Richland, WA 1975.

21. M. W. Chase, et al., JANAF Thermochemical Tables, 1975 Supplement, J. Phys. Chem. Ref. Data 4: 102-3, 1975.

22. D. F. Smith, et a1., U.S. Bur. Mines, RI-6316, 1963.

23. R. I. Efremova and E. V. Matizen, Izv. Sib. Akad. Nauk SSSR, Ser. Khim. Nauk, (1), 3, 1970.

24. V. R. Belosludov, R. I. Efremova, and E. V. Matizen, Sov. Physics. Solid State 16: $847-51,1974$.

25. H. Kojima, S. G. Whiteway, and C. R. Masson, Can. J. Chem. 46: 2968-72, 1968 .

26. G. Petit and F. Delbove, Compt. Rend. 254: 2388, 1962.

27. B. Porter and E. A. Brown, J.Amer. Ceram. Soc. 45; 49, 1962.

28. V. T. Berezhnaya and G. A. Bukhalova, Russ. J. Inorg. Chem. (English Trans.)5: 445, 1960, Zr. Neorgan. Khim. 5 (4):925, 1960.

29. H. T. Fullam, Pacific Northwest Labortory Quarterly Report to USERDA Advanced Nuclear Energy Systems, Space and Special Purposes Divisions for July-September 1975, BNWL-2845-16, Pacific Northwest Laboratory, Richland, WA, 1975.

30. Gmelin's Handbuch der Anorganischen Chemie, Strontium-System No. 29, Weenheim/Bergstrasse, 1960. 
31. I. G. Kipov and S. N. Zadumkin, Russ. J. Phy. Chem. (English Trans.) 46:063-64, 1972.

32. H. E. Swanson, et a1., Nat. Bur. Stand. Rep. 3684, 1954.

33. H. T. Fullam, The Solubility and Dissoluton Behavior of ${ }^{90} \mathrm{SrF}_{2}$ in Aqueous Media, BNWL-2101, Pacific Northwest Laboratory, Richland, WA, 1976

34. R. C. Pastor and K. Arita, Mat. Res. Bul1. 10:493-500, 1975.

35. C. Raisin, J. M. Kerger, S. Robin-Kandare, G. Kroll, and A. Amamou, J. Phys. C. Solid. St. Phy. 13, 1835-44, 1980.

36. M. Nisar and S. Robin, Pakistan J. Sci. Ind. Res. 17: 49-54, 2974.

37. C. E. Derrington, A. Linder, and M. O'Keeffe, J. Solid State Chem. 15: $171-74,1975$.

38. J. Oberschmidt and D. Lazarus, Phys. Rev. B $21: 5823-34,1980$.

39. C. Andeen, J. Fontanel1a, and D. Schulele, J. App. Phys. 42; 216219, 1971.

40. G. Y. Chin, M. L. Green, L. G. VanVitert, and W. A. Hargreaves, J. Mat. Sci. 8; 1421-25, 1973.

41. F. A. Horrigan and T. F. Deutsch, Research in Optical Materials and Structures for High Power Lasers, Raytheon Research Division, Waltham, MA., Second Quarterly Report - December 1970, U.S. Army Missile Command, Redstone Arsenal, Alabama, ARPA Order No. 1180.

42. K. E. D. Wapenaar, and J. Schoonman, J. Solid State Chem. 25:31-37, 1978.

43. H. Bontinck, Physica. 24: 650-654, 1958.

44. H. T. Fullam, Pacific Northwest Laboratory Quarterly Report on Space Nuclear Systems Program for period ending June 30, 1974, BNWL-2845-1, Pacific Northwest Laboratory, Richland, WA, 1975.

45. H. T. Fullam, Quarterly Report on the Strontium Heat Source Development Program and the Terrestrial Radioisotope Applications Program, Advanced Nuclear Systems and Materials Production Division for October-December 1977, PNL-2845-37, Pacific Northwest Laboratory, Richland, WA, 1978. 
46. H. T. Fullam, Containment of Strontium Fluoride at $800{ }^{\circ} \mathrm{C}$-Supplemental Screening Tests, BNWL-2259, Pacific Northwest Laboratory, Richland, WA 1977.

47. H. T. Fullam, Compatibility of Strontium-90 Fluoride with Containment Materials at Elevated Temperatures, PNL-3833, Pacific Northwest Laboratory, Richland, WA 1981.

48. H. T. Fullam, Compatibility Testing of the WESF ${ }^{90} \mathrm{SrF}_{2}$ Storage Capsule at $800^{\circ} \mathrm{C}$, PNL-3433, Pacific Northwest Laboratory, Richland, WA 1980. 
PNL-3846

$U C-4, U C-23$

\section{DISTRIBUTION}

No. of

Copies

OFFSITE

A. A. Churm

DOE Patent Division

9800 S. Cass Avenue

Argonne, IL 60439

F. E. Coffman

DOE Office of Advanced Nuclear

Systems and Projects, NE-540

Washington, DC 20545

G. L. Bennett

DOE Space and Terrestrial

Systems Division, NE-543

Washington, DC 20545

B. J. Rock

DOE Space and Terrestrial

Systems Division, NE-543

Washington, DC 20545

J. J. Lombardo

DOE Space and Terrestrial

Systems Division, NE-543

Washington, DC 20545

25 W. C. Remini

DOE Operations and By-Products Utilization Division, NE-320 Washington, DC 20545

J. E. Dieckhoner

DOE Operations and By-Products

Utilization Division, NE-321

Washington, DC 20545

D. J. McGoff

DOE Projects and Technology

Division, NE-323

Washington, DC 20545
No. of

Copies

W. Savage

DOE Space and Terrestrial

Systems Division, NE-543

Washington, DC 20545

J. M. Maddox

DOE

E201

Washington, DC 20545

C. A. Cooley

DOE Waste Management Division

Washington, DC 20545

G. Oertel

DOE Waste Management Division

Washington, DC 20545

D. C. Davis, Jr.

DOE Oak Ridge Operations Office

P. 0. Box E

Oak Ridge, TN 37830

W. T. Goldston

DOE Savannah River Operations Office

P. 0. Box A

Aiken, SC 29801

27 DOE Technical Information Center

L. L. Jardine

Argonne National Laboratory

9700 South Cass Avenue

Argonne, IL 60439

R. T. Huntoon

E. I. duPont deNemours and Company

Savannah River Laboratory

Aiken, SC 29801 
No. of

Copies

E. H. Cooke-Yarborough

Electronics and Applied Physics Division

Building 347.3, AERE Harwell

OXfordshire OXI1 ORA

GREAT BRITAIN

S. E. Bronisz

Los Alamos Scientific Laboratory

P. O. Box 1663

Los Alamos, NM 87544

J. S. Sivinski

Division 4535

Sandia Laboratories

Albuquerque, NM 87185

W. T. Cave

Monsanto Research Corporation

Mound Laboratory (DOE)

Nuclear Operations

P. 0. Box 32

Miamisburg, $\mathrm{OH} 45342$

H. H. Musselman, Tech. Dir.

Department of the Army

Headquarters, U.S. Army

Facilities Engineering Support Agency

Fort Belvoir, VA 22060

Officer in Charge

J. H. Vogt

Naval Nuclear Power Unit

Code 70

Port Hueneme, CA 93043

H. V. Weiss

Code 631

Naval Ocean Systems Center

San Diego, CA 92152
No. of

Copies

2 A. A. Arcuni

Naval Facilities Engineering Command

Office of Special Assistant Nuclear Programs (04N)

200 Stovall Street

Alexandria, VA 22332

K. W. Haff

Cak Ridge National Laboratory

Oak Ridge, TN 37830

J. Hammond

Oak Ridge National Laboratory

Oak Ridge, TN 37830

J. R. Keiser

Oak Ridge National Laboratory

Oak Ridge, TN 37830

3 E. Lamb

Oak Ridge National Laboratory

Oak Ridge, TN 37830

2 B. J. Tharpe

General Electric Company

Space Division

P. 0. Box 8661

Philadelphia, PA 19101

2 R. Englehart

NUS Corporation

4 Research Place

Rockville, MD 20850

2 P. Dick

Teledyne Energy Systems

110 W. Timonium Road

Timonium, MD 21093

C. C. Silverstein

Westinghouse Astronuclear Laboratory

P. 0. Box 10864

PIttsburgh, PA 15236 
No. of

Copies

\section{ONSITE}

4 DOE Richland Operations
D. Bogon
R. E. Gerton
H. A. House
H. E. Ransom

11 Rockwell Hanford Operations

H. H. Hopkins

J. D. Kaser

E. J. Kosiancic

T. H. May

K. J. Pascoe

I. E. Reep

P. F. Shaw

J. P. Sloughter

C. R. Stroup

D. D. Wodrich

G. D. Wright

35 Pacific Northwest Laboratory

H. T. Fullam (25)

R. E. Nightingale

L. D. Perrigo

H. H. Van Tuyl

Technical Files (5)

Publishing Coordination (2) 